\title{
Behind the seen : unconscious perception of objects, faces and emotions
}

Citation for published version (APA):

Seirafi, M. (2015). Behind the seen : unconscious perception of objects, faces and emotions. [Doctoral Thesis, Maastricht University]. Maastricht University. https://doi.org/10.26481/dis.20151030ms

Document status and date:

Published: 01/01/2015

DOI:

10.26481/dis.20151030ms

Document Version:

Publisher's PDF, also known as Version of record

\section{Please check the document version of this publication:}

- A submitted manuscript is the version of the article upon submission and before peer-review. There can be important differences between the submitted version and the official published version of record.

People interested in the research are advised to contact the author for the final version of the publication, or visit the DOI to the publisher's website.

- The final author version and the galley proof are versions of the publication after peer review.

- The final published version features the final layout of the paper including the volume, issue and page numbers.

Link to publication

\footnotetext{
General rights rights.

- You may freely distribute the URL identifying the publication in the public portal. please follow below link for the End User Agreement:

www.umlib.nl/taverne-license

Take down policy

If you believe that this document breaches copyright please contact us at:

repository@maastrichtuniversity.nl

providing details and we will investigate your claim.
}

Copyright and moral rights for the publications made accessible in the public portal are retained by the authors and/or other copyright owners and it is a condition of accessing publications that users recognise and abide by the legal requirements associated with these

- Users may download and print one copy of any publication from the public portal for the purpose of private study or research.

- You may not further distribute the material or use it for any profit-making activity or commercial gain

If the publication is distributed under the terms of Article $25 \mathrm{fa}$ of the Dutch Copyright Act, indicated by the "Taverne" license above, 


\section{BEHIND THE SEEN}

UNCONSCIOUS PERCEPTION OF OBJECTS,

FACES AND EMOTIONS 
(c) Mehrdad Seirafi, Maastricht 2015. All rights reserved. No part of this publication may be reproduced, stored in a retrieval system or transmitted in any form or by any means, electronic, mechanical, photocopying, recording or otherwise, without prior written permission of the publisher.

Cover

Production

ISBN
Mehrdad Seirafi (credits: Tim Bower, Mondrian)

Proefschriftmaken.nl

ISBN 978-94-6203-940-7 


\title{
BEHIND THE SEEN
}

\section{UNCONSCIOUS PERCEPTION OF OBJECTS, FACES AND EMOTIONS}

\author{
DISSERTATION \\ To obtain the degree of Doctor at Maastricht University, \\ on the authority of the Rector Magnificus, Prof. Dr. L.L.G. Soete, \\ in accordance with the decision of the board of Deans, to be defended in public \\ on Friday, October 30, 2015, at 14:00.
}

By

Mehrdad Seirafi

Born on 21 March 1982

Tehran, Iran 


\section{SUPERVISORS}

Prof. Dr. Beatrice de Gelder

Prof. Dr. Peter De Weerd

\section{ASSESSMENT COMMITTEE}

Prof. Dr. Bernadette Jansma (Chair)

Dr. Valerie Goffaux (Université Catholique de Louvain, Belgium)

Prof. Dr. Alain Ptito (McGill University, Canada)

Dr. Joel Reithler 


\section{Table of Contents}

$\begin{array}{llr}\text { Chapter } 1 & \text { General introduction } \\ \text { Chapter } 2 & \text { Seeing Emotions in the Objects }\end{array}$

Chapter $3 \quad$ Attribute-Selective Adaptation $\mathbf{5 1}$

Category-specific effect of adaptation on faces and objects

$\begin{array}{lll}\text { Chapter } 4 & \mathbf{8 1}\end{array}$

Suppression of face perception during saccadic eye movement

$\begin{array}{lll}\text { Chapter } 5 & \text { Faceless Emotions } & 101\end{array}$

Emotion categorization in the absence of face detection

$\begin{array}{lll}\text { Chapter } 6 & \text { Audiovisual Blindsight } & 119\end{array}$

Audiovisual learning in the absence of primary visual cortex

$\begin{array}{lll}\text { Chapter } 7 & \text { Cognition and Metacognition } & 133\end{array}$

Technical challenges in assessing near-zero measures of cognition and metacognition

$\begin{array}{lll}\text { Chapter } 8 & \text { General Discussion } & 151\end{array}$

$\begin{array}{ll}\text { Valorization } & 167\end{array}$

Acknowledgements 173

$\begin{array}{ll}\text { Publications } & 181\end{array}$

$\begin{array}{ll}\text { About the Author } & 182\end{array}$ 

CHAPTER 1

\section{GENERAL \\ INTRODUCTION}




\section{Preface}

Faces are the most important visual stimuli, necessary for our social interactions. They simultaneously convey several biologically-significant signals such as identity, gender, emotional expression, etc. As a result, our central nervous system has mastered -in the course of evolution- the ability to process this category of visual objects in an extremely rapid and effortless manner.

A large number of behavioral studies have provided significant insights into the nature of face perception. Nevertheless, the underlying mechanism for such a powerful computational capability is still far from completely understood. Any advancement in this respect not only gives a better understanding on how the brain functions but also brings us one step closer to building biologically-inspired machines with face recognition abilities.

To know how face perception works, we first need to know how object recognition as a whole works, since both faces and objects share the same central challenge: they both require to distinguish among often similar visual forms despite the significant variations in position, illumination, occlusion, etc. of the forms. Thus, to give an overview on our current understanding of face perception, first, I give a summary of our present knowledge about the more general problem of "object recognition".

Next, I will discuss certain aspects of face recognition which makes it "special" compared to the other forms of object recognition. For this, I will present several lines of research highlighting the differences in the processing of face stimuli compared to the processing of other objects. For this I will refer to the research on whether the processing of faces differs from that of the other objects. Subsequently, I present the current structural and functional models of face perception and the evidence supporting them.

Finally, at the end of this chapter, I give an overview of unconscious perception and explain how the unconscious perception of objects in general and faces in particular differs from the conscious perception. This is especially relevant since there is very little known about the unconscious processing of stimuli within the current models of face perception. In the next research chapters, I will mainly present my research questioning the present models of face and object recognition in the context of unconscious perception. 


\section{1| Object Recognition}

The detection and recognition of meaningful objects in complex environments is a crucial skill that underlies a wide range of behaviors, from identifying preys and predators to reading words in this text, and from recognizing edible foods to finding familiar faces in a crowd. In humans and primates, these processes operate effortlessly, quickly and automatically (Biederman, 1987; Potter, 1976). Thus, object recognition is one of the key evolutionary means of survival, engaging more than half of the non-human primate brain (Felleman \& Van Essen, 1991). In particular, the recognition of coherent meaningful objects involves integration of components at different levels of visual complexity, beginning with local contours at primary visual areas to complex objects at higher level modules. These representations can accommodate environmental variations such as changes in viewpoint, size, position, background clutter, etc.

\section{Neurophysiology of Object Recognition}

Since the early discoveries in the 1970 s and 1980s (Desimone \& Gross, 1979; Mishkin, Ungerleider, \& Macko, 1983), various fields of neuroscience research now provide converging evidence that the neural substrates involved in object recognition are mainly located in the ventral visual stream (Haxby et al., 2001; Martin, Wiggs, Ungerleider, \& Haxby, 1996). The main underlying anatomical mechanism appears to consist of a number of processing stages that are organized in a roughly hierarchical order: the information is projected from the retina to early primary visual cortex ( $\mathrm{v} 1$ ) which has small receptive fields (RFs) (R Gattass, Gross, \& Sandell, 1981; Hubel \& Wiesel, 1962). Then the information is sent from V1 to mid-level cortical areas with medium RFs (R. Gattass, Sousa, \& Gross, 1988), ending up at the Inferior Temporal Cortex (IT) in monkeys (Gross, Bender, \& Rocha-Miranda, 1969; Gross, Rocha-Miranda, \& Bender, 1972) and to its human homologues Lateral Occipital Complex (LOC) (Kourtzi \& Kanwisher, 2000) and the fusifurm gyrus (FG)(Clarke \& Miklossy, 1990; Sergent, Ohta, \& MacDonald, 1992). As a result, the high-level representations are relatively invariant to the changes in position and size (Afraz \& Cavanagh, 2008; Kovacs, Zimmer, Harza, Antal, \& Vidnyanszky, 2005; Lueschow, Miller, \& Desimone, 1994). 
Distributed representations of objects. Studying the properties of object recognition in the past two decades has provided important information how this system works. One of the important findings is that the cells in area TE of the IT cortex of the monkey brain respond selectively to various complex object-features (K. Tanaka, 1993). Moreover, the neurons that respond to similar features are clustered in a columnar like fashion (K. Tanaka, 1996). Furthermore, these neurons represent complex visual objects using a sparse population code (Kourtzi, Betts, Sarkheil, \& Welchman, 2005). In humans -using non-invasive techniques- similar patterns of object recognition have been revealed. Numerous $\mathrm{fMRI}$ studies have shown a similar distributed representation of objects in the human ventral pathway. For example, studies contrasting faces, houses and chairs have identified differential activation maps bilaterally in ventral temporal and ventral occipital cortices and superior temporal sulcus (Ishai, Ungerleider, \& Haxby, 2000; Ishai, Ungerleider, Martin, Schouten, \& Haxby, 1999). Such studies propose that object representation in the ventral visual pathway is done by a distributed representation of information about the form and not by small, highly selective cortical patches. More recently, combining $\mathrm{fMRI}$ and single cell recording the existence of several face-selective patches with more than $95 \%$ selectivity to the face stimuli in different cortical regions has been established (Tsao et al. (2006, 2008)).

\section{Object Recognition Models}

Being inspired by the anatomical findings, models of object recognition have been updated in the past decades. Marr and Nishihara (Marr \& Nishihara, 1978) proposed a hierarchical structure of bottom-up processing, which was the dominant view until recently. In their model simple feature detectors (similar to the simple cells in the early visual areas) are at the bottom of the processor and as the information reaches upper levels in the hierarchy the complexity of the features increase (comparable to the neurons in the IT cortex). In summary, object recognition can be roughly reduced to three processing stages (Humphreys \& Price, 2001), after the processing of basic component such as depth, form, color, etc.:

Figure-ground segregation. This stage is the result of grouping the basic components and provides information on distinct edges to the visual form. 
Categorization. This stage refers to the matching of the visual representation with the structural descriptions stored in the memory. Structural descriptions are the specifications of the components of the objects and their interrelations.

Identification. At this stage, recognition by semantic attributes to the visual representations takes place.

The more recent models -unlike the traditional ones- propose a notstrictly-hierarchical structure (Bar et al., 2006) by connecting the coarse representations at early processing stages to the highest level of hierarchy (prefrontal cortex (PFC)). PFC, in-turn, projects to the IT neurons, activating the relevant object representations based on the most likely candidates. This minimizes the number of object representations evoked by the bottom-up information flow.

\section{2 | Face Perception}

\section{Are Faces Special?}

Whether faces are special or not has been one of the hot topics in the field of face perception in the past decades. The idea that faces require special neural mechanisms has been countered with the idea that faces just represent a case in which perception has been enhanced due to training and resulting expertise. Indeed, among all possible objects humans are most expert at face recognition (Carey, De Schonen, \& Ellis, 1992; Gauthier, Skudlarski, Gore, \& Anderson, 2000). Like dog watchers have expertise in identifying dogs and birdwatchers exquisite ability to distinguish birds, face experts need to identify individual faces rapidly and accurately (J. W. Tanaka, 2001). This viewpoint emphasizes expertise in within-category identification to be the content of what is coded in face-selective areas (Gauthier, Behrmann, \& Tarr, 1999; Gauthier, Tarr, Anderson, Skudlarski, \& Gore, 1999). It is important to note that this account is not necessarily in contradiction with the face specialness viewpoint, at least in the majority of the population with no other visual expertise except for the faces. Here, I give a brief overview of the studies that led to the concept of specialized mechanisms for face detection. 
Neural correlates of face detection. How to detect faces against a noisy background has been one of the challenges in various fields of research for the past three decades. The term face detection was first introduced in the field of computer vision together with word detection and line detection. However, the exact definition has changed in the past decades and there is still no consensus on its exact meaning. In vision research, Owsley et al (1981) first adopted the concept of face detection in a study investigating the relationship between aging and low-contrast vision using spatial frequency filtered face stimuli with different contrasts. In the same year, Bruce et al. found the first face selective neurons in the monkey inferior temporal cortex, and was followed by other studies discovering neurons selective for the face as a whole in addition to neurons selective for face parts (Bruce, Desimone, \& Gross, 1981; Perrett, Rolls, \& Caan, 1982). They also showed that some of the face selective neurons (around 40\%) respond equally well for different view-points of the face stimuli. In the later years, neurons responsive to different patterns, such as irregular edges, faces and combinations of red and white stimuli and hands, were also found (Desimone, Albright, Gross, \& Bruce, 1984). Later, Purcell and Stewart $(1986,1988)$ defined the face detection effect (FAE) in the context of visual perception as the ability to discriminate whole faces versus rearranged facial features. These studies suggest a neural network which gets activated by exposure to the face stimuli.

Face-specific processes. Except for the neurophysiological data on face detection, the main evidence for specialness of face perception comes from two major sources: (a) the behavioral differences in the recognition performance of faces and non-face objects; (b) the neuropsychological findings revealing a double dissociation in deficits for face and object recognition. These aspects will be discussed in more details below.

Behavioral evidence. The behavioral effect on performance in face recognition tasks of specific face manipulations is the first piece of information suggesting specialness of face processing. One manipulation is presenting the faces upside-down, which leads to a dramatic decrease in viewer's performance in a face recognition task (Yin, 1969). This is especially interesting since all the global low-level features are kept constant in such a manipulation. The other major characteristic of face perception is being sensitive to the contrast polarity in which the image is presented. More specifically, a significant performance drop in face recognition task has been shown for the negative images of faces 
in which light areas appear dark and vice versa (Kemp, McManus, \& Pigott, 1990). Note that these studies also showed that the two manipulations are much less detrimental for the recognition of the non-face objects than that for the recognition of faces.

Neuropsychological findings. The other piece of evidence for face specialness comes from studies of patients with focal brain damage. One line of evidence comes from neuropsychological findings in patients with object agnosia (unable to recognize objects) but without face agnosia. For example, several studies on patient $\mathrm{CK}$, exhibited his severe deficit in object recognition performance, despite his perfect performance in face recognition tasks (Moscovitch, Winocur, \& Behrmann, 1997). Studies of this patient suggest that face recognition is not simply the final stage of the object recognition system but rather a distinct process taking place at an early stage in the visual system, or in partially different pathways. Other researchers demonstrated that this dissociation can be made in the other direction as well (MCCARTHY \& Warrington, 1986). For this, they tested two groups of patients - each with diminished performance in either face or non-face recognition tasks. Their results showed a clear double dissociation of the deficits for face- and object recognition.

\section{Structural and Functional Models for Face Perception}

Early models of face perception were proposed shortly after the discovery of face-selective neurons in monkeys. Inspired by physiological data and inspired by general object recognition models, Bruce and Young (1986) proposed a cognitive model for face perception (Figure $1 \mathrm{~A}$ ). The most common views on how face recognition takes place are relying on this model: the face stimuli are first processed in a central division of "structural encoding" which first serially generates view-centered and expression independent descriptions of the face. This module of structural encoding produces two kinds of output. On the one hand it projects to processors for facial speech analysis, expression analysis, etc. On the other hand, it projects to separate processors for face recognition, for the determination of identity, and other related functions. The important characteristic of this model is its emphasis on the distinction between processes involved in the recognition of identity and the ones involved in recognition of emotion and speech. This model was later updated to a functional model taking into account the research on neural substrates of face processes (Haxby, 
Hoffman, \& Gobbini, 2000). Haxby et al.'s functional model includes a core system, comparable to the structural encoding subsystem in Bruce and Young's model. The core system consists of an early perception of facial features in inferior occipital gyri, followed by two parallel units processing the changeable in superior temporal sulcus, and the invariant aspects of the face in lateral fusiform gyrus. The changeable aspects send input to the prelexical speech perception in the auditory cortex and the emotion recognition system (limbic system and insula); the invariant aspects feed the person identification in the anterior temporal cortex.

Subcortical face processing. Recent behavioral, functional imaging and neuropsychological findings suggest a subcortical route for face perception. Some researchers proposed that the subcortical route does not simply respond to the top-down influence from cortex, but that it modulates cortical processing units for faces and other socially-relevant stimuli. The evidence suggesting such involvement comes from three domains: infant data, spatial frequency manipulations, and neuropsychological studies.

Infant studies. Two converging lines of research on face perception in newborns support the hypothesis of subcortical routes for face processing. One line of evidence comes from the studies showing that human infants have a preference for face-like stimuli, despite their relatively immature visual cortices, whereas the subcortical route seems to be more mature (Atkinson et al., 2001; Johnson, Dziurawiec, Ellis, \& Morton, 1991; Webster, Bachevalier, \& Ungerleider, 1994). The second line of evidence supporting the view of a subcortical route for newborns' face preference comes from other species: almost all vertebrates can recognize biologically relevant stimuli at, or shortly after birth (Lagercrantz, Hanson, Ment, \& Peebles, 2010). In birds, rodents and primates, the subtelencephalon has been established to play a role in the processing of such survival-relevant object recognition (Horn, 2004; Sewards \& Sewards, 2002).

Spatial frequency channels. The spatial frequency spectrum of the observed objects influences significantly the way they are processed in the visual system. Previous research has shown that a relatively small range of frequencies -between 8 and 16 cycles, has a pronounced effect on the visibility and ability to identify face images (Costen, Parker, \& Craw, 1996; Näsänen, 1999). Specifically, if the information content of the face stimuli in this frequency range is disrupted, identification or categorization performance 
drops dramatically but facial expressions are still visible. Furthermore, other studies suggest a coarse to fine strategy in processing of the face images: in brief exposures of masked faces, only the low-frequency content is processed in the face-preferring regions, whereas the high-frequency content is the dominant one in the long exposures (Goffaux et al., 2011). Such studies suggest a rapid coarse representation of the low-frequency information followed by a detailed representation of the faces. Another imaging study showed that faceselective cortical areas are activated by only high- and mid- spatial frequency spectra of the faces and not by the low frequencies (Vuilleumier et al. (2003)). They also demonstrated that subcortical structures such as pulvinar, amygdala and superior colliculus get activated by the low spatial frequency spectrum of the faces and not by the high ones.

Face detection process and dual route model. One intriguing finding regarding face perception is the finding called inversion superiority in prosopagnosic patients. Inversion superiority refers to the higher recognition performance seen in a (face or object) recognition task when the patient is viewing the images in a non-canonical way (e.g. upside-down). This effect was first observed both in some prosopagnosic patients in face recognition tasks (Farah, Wilson, Drain, \& Tanaka, 1995) where they exhibited a higher performance for inverted faces compared to the upright ones. They concluded that the code of inverted faces at least partially overlaps with the object recognition system. Furthermore, a similar study in patients with agnosia using face and object recognition tasks (de Gelder \& Rouw, 2000) showed the same pattern. Interestingly, the prosopagnosics demonstrated intact face detection performance.

Based on this paradoxical inversion superiority effect, de Gelder and Rouw (2001) proposed that face detection and face recognition may use entirely different neural resources (Figure $2 \mathrm{~A}$ ). In their model, face perception consists of two systems. Some of the main properties of the two systems are as follows. On the one hand, the system involved in face detection is thought to operate fast, to be category specific (unique to faces), and to use coarsegrained representations. This system is thought to require only limited stimulus exposure and is thought to be ontogenetically primitive (present in newborns). The other system which is involved in face identification, is slower, requires longer exposures and is thought to generate more fine-grained representations, Moreover, it is assumed to be ontogenetically complex. The same model was later adopted by Johnson (2005) in his model for subcortical 
face processing (Figure 2B). In his model, face detection takes place in superior colliculus (SC), pulvinar and amygdala.

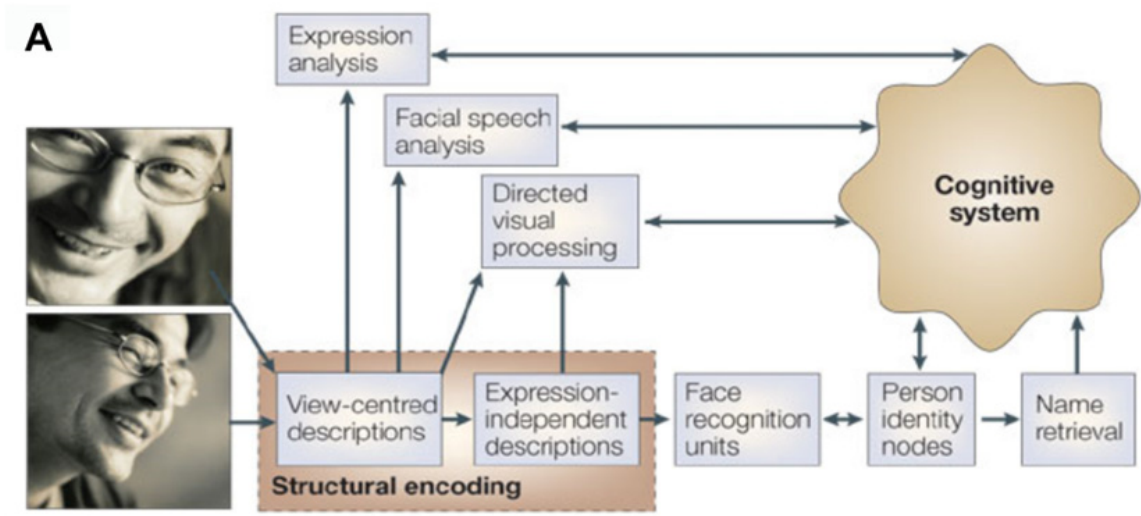

B

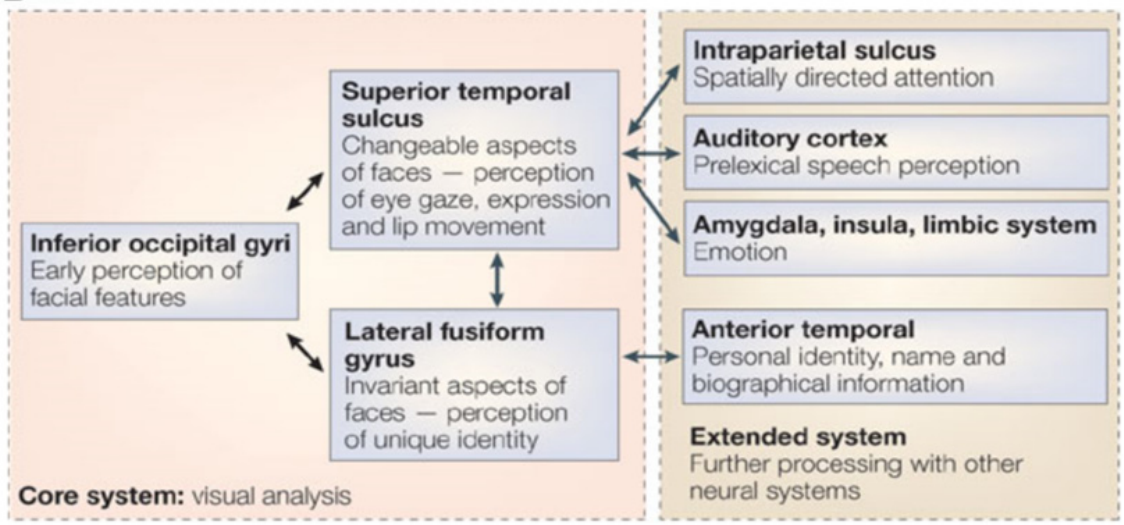

Figure 1 Summary of the conventional face perception models (Calder \& Young, 2005). (a) Cognitive model for face perception (Bruce and Young, 1986); (b) Functional model for face perception by Haxby et al. (2000).

An important point in all face perception models is that the input is always assumed to be a face. In other words, there is no prediction for any of the above-mentioned models how the face system works if the input stimulus is anything but a face. I will return to this point in the next chapter. 
A

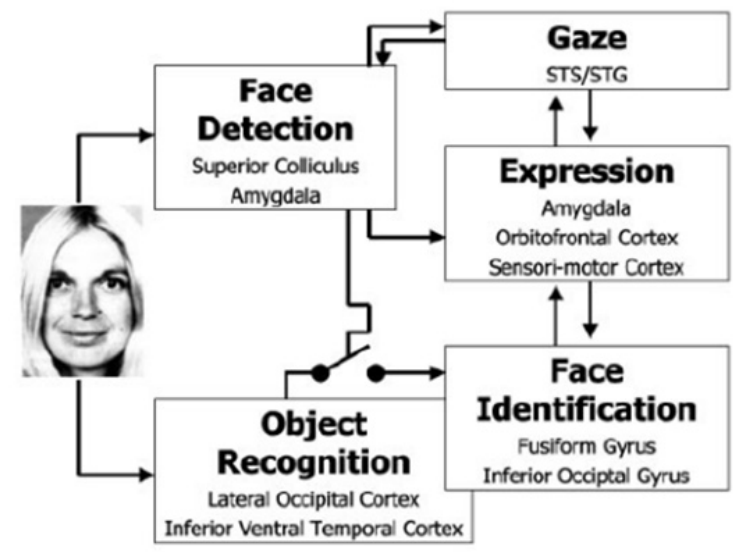

B

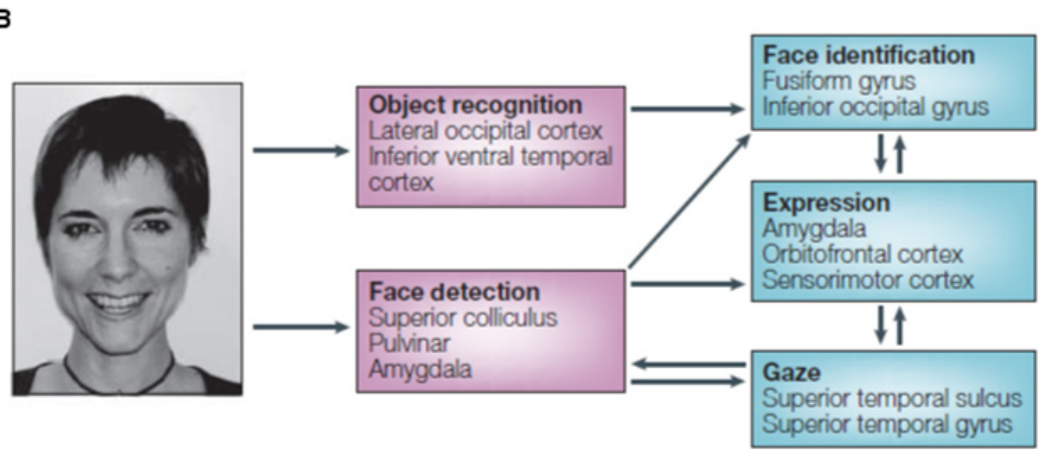

Figure 2 Dual route models for face perception: (A) the original model by de Gelder et al. (2003); (B) the extension of de Gelder and Rouw's dual route model for face processing proposed by Johnson (2005).

\section{Specific Process of Emotion Recognition in the Present Models}

As mentioned before, the conventional dominant view is that categorization of the stimulus as a 'face' is required in order to achieve emotional expression recognition (Bruce \& Young, 1986; Haxby et al., 2000). In alternative models in which face detection is thought to depend on separate neural substrates, the assumption is that emotional expressions can be extracted very efficiently and rapidly, even prior to the conscious experience of a face. The ability of detecting emotions in faces prior to the conscious categorization of the presented stimulus as a face hence has become a crucial prediction of theories assigning 
separate processing pathways to face detection. However, studies arguing for relative independence between face perception and expression recognition so far have failed to exclude the possibility that some aspect of the stimulus was in fact consciously detected, and was used to guess the emotional expression (Morris, DeGelder, Weiskrantz, \& Dolan, 2001). This is an important point that I will return to in Chapter 5 .

\section{3| Conscious versus Unconscious Perception of Objects and Faces}

Perception without awareness has been a topic of interest for more than a century. The importance of this question dates to the traditional viewpoint about the relationship among perception, feeling and action, which in-turn is rooted in the more fundamental philosophical questions about being and freewill. This perspective implies that any perception, feeling or action requires the awareness of perceiving. Given this belief, any evidence of perception without awareness challenges the traditional viewpoint about the central role of conscious awareness for any perception and action.

One of the first studies challenging the traditional view that awareness is a prerequisite for perception dates back to 1898 , when Sidis presented visual stimuli from two categories of numbers and letters too far away from the participants to be clearly seen (Sidis, 1898). The participants reported no awareness about the content of the stimuli except a blurry print. Sadis asked the participants to guess the category of the stimuli in a two-alternative forced choice task. The results showed that the participants could successfully discriminate between the two categories despite not being aware of their perception.

Another relevant field of research to unconscious perception in the past few decades is blindsight. Blindsight is defined as the visual capacity of people who are cortically blind due to lesions in their primary visual cortex (V1), to respond to visual stimuli without acknowledged awareness (Weiskrantz, 1986). The phenomenon has been known in animal studies in monkeys in cats. The early research showed that the animals can perform successfully in the visual tasks without primary visual cortex, whereas humans with such lesions reported unawareness of the stimuli. The question was raised later if those animals actually perceived the stimuli consciously or not, i.e. if certain pieces of information can lead to action without reaching awareness. In cats, visual 
capacities after $\mathrm{V}_{1}$ lesions do not involve blindsight. Their visual behavior after $\mathrm{V} 1$ lesions is close to normal, likely because of the strong extra-geniculate input to extrastriate cortex (Doty, 1971; Sprague, De Weerd, Xiao, Vandenbussche, \& Orban, 1996). In monkeys with V1 lesions (Humphrey \& Weiskrantz, 1967) normal visual behavior is absent, but some residual discrimination does persist. Whether or not this was due to spared islands of $V_{1}$ tissue, allowing some partial conscious perception was addressed by a few studies in patients with defects in the occipital lobe. In humans, it may be easier to probe conscious experience (or the absence thereof). The patients in blindsight studies reported to be blind despite exhibiting residual visual processing in forced choice tasks (Pöppel, Held, \& Frost, 1973; Weiskrantz, Warrington, Sanders, \& Marshall, J., 1974). Interestingly, these patients showed physiological signs of unconscious processing, such as pupil size difference, as a reaction to contrast-modulated gratings (Weiskrantz, Cowey, \& Le Mare, 1998). Other studies also provided evidence of the priming effect in the blind field (Marzi, Tassinari, Aglioti, \& Lutzemberger, 1986; Rafal, Smith, Krantz, Cohen, \& Brennan, 1990). From the above, it is clear that good measures of awareness are important tools in blindsight research as well as in other research probing the possibility for unconscious perception.

\section{Measures of Awareness}

Subjective measures of awareness are based on participant's reports of whether or not they perceive the stimulus. The subjective threshold is the transition level of information content at which the participants begin to perceive the stimuli. By contrast, objective measures of awareness are quantified in a forced-choice procedure, in which participants must choose which stimulus was presented from two (or more) options given to them. The objective threshold is the transition level from chance level performance to significantly above-chance. The amount of information can be manipulated by changing the properties of the stimuli parametrically; for example, by varying the duration of presentation for the visual stimuli or by changing the contrast of the images.

Previous research has demonstrated that the subjective threshold follows the objective threshold by 30 to 50 milliseconds in certain experimental conditions (Cheesman \& Merikle, 1984). This indicated that participants were able to objectively discriminate between stimuli while they were still not at the subjective threshold. In other words, the minimal information to reach the 
objective threshold was less than the minimal information required to reaching the subjective threshold.

\section{Signal Detection Theory: Technical Approach to Perception}

The judgment of a participant in detection and discrimination tasks depends on two distinct factors. The first factor is the actual perceptual sensitivity to the target stimuli displayed against the non-target stimuli. The other factor is the tendency to choose one of the responses over the others, independent of the sensitivity. Signal detection theory (SDT) has been developed to solve this problem by discerning the subject's sensitivity from the response bias (Green \& Swets, 1966; Macmillan \& Creelman, 2004). The simplest measure of sensitivity within the signal detection theory framework is $d^{\prime}$ (d-prime). The $d^{\prime}$ is calculated by finding the distance of the normalized (inverse of the cumulative distribution function) values of the hit and the false alarm rates. An important property of such a measure is its independence of the actual subjective criterion used by the subject. The underlying assumption for calculating this measure is a normal distribution of the target and non-target. Zero value for $d$ ' means no sensitivity in discriminating the target from the non-target. The higher values mean the more discernibility of the two. However, there is no consensus on how to relate the objective value of sensitivity to conscious perception. Some researchers proposed the $d$ ' of zero as complete lack of conscious awareness and $d$ ' values between 0 and 0.5 as subliminal conscious awareness (Macmillan \& Creelman, 2004). On the other hand, some researchers have proposed the subjects' ability to distinguish their own correct and incorrect responses based on SDT -known as metacognition- as a measure of the subjective threshold. However, most researchers still rely on participants' report on seen or not seen the stimuli to measure the subjective threshold, and recent models assume $d$ ' and subjective ratings to reflect distinct phenomena (King \& Dehaene, 2014).

\section{Unconscious Perception of Emotions and Affective Blindsight}

Traditionally, conscious feeling has been assumed to be the core of emotion. The root of this assumption goes back to the renowned article by James (1884) on emotions, where he defined emotion as the perception of bodily changes forming a conscious feeling (James, 1884). He argued that consciousness is exactly what makes the feeling distinct from other sorts of mental states. Later, 
Freud also explained emotion as a conscious state whereby the underlying causes might not be consciously perceived (Freud, 1922).

However, recent research challenges this traditional view by proposing the possibility of "unconscious emotions". One line of research supporting this has focused on demonstrating so-called sub-liminal emotions. For example, Monahan, Murphy, and Zajonc (2000) showed that visual stimuli with emotional content, which were suppressed from conscious perception, could change the mood of the viewers at the end of the experimental session. Other researchers carried out similar experiments using subliminal negative and positive emotional stimuli, such as snakes and spiders, in participants with phobia (Öhman, Flykt, \& Lundqvist, 2000). They showed that the mood of the participants could clearly be influenced by the subliminal stimuli.

Moreover, recent imaging studies have investigated the underlying conscious and unconscious processing routes of emotional stimuli (Öhman, 2005). Öhman showed that both masked fearful facial stimuli and unseen images of threatening animals activated the amygdala. Moreover, the study demonstrated that if the experimental conditions did not allow conscious processing, the amygdala response to fear stimuli, including faces and threatening animals, was elevated and a cortical network, including anterior cingulate cortex and the anterior insula, was activated. However, if the experimental conditions permitted conscious processing to take place, the fearful face stimuli did still activate the same network while the fear-relevant (e.g. spider for the ones with phobia of spiders) did not activate the same network anymore. Instead, there was a rise in the orbitofrontal and dorsolateral network which is supposed to inhibit the amygdala activity. The amygdala activity is mediated by a subcortical pathway including superior colliculus and pulvinar, which operates on low-spatial frequency information.

The first report of a blindsight patient who could reliably discriminate the emotional content of face stimuli presented in the blind field dates back to a study by de Gelder, Vroomen, Pourtois, \& Weiskrantz (1999). They demonstrated that a well-known blindsight patient, patient GY, could successfully (above chance) guess the facial expression of still images and short videos of different emotions that were presented in his blind visual field. This finding was of great importance since, until then, all research on such patients were merely focused on very basic visual objects, such as gratings, moving dots and colors (Weiskrantz, 1986; Weiskrantz, Warrington, Sanders, \& Marshall, J., 1974). This was a manifestation of a visual processing capability much more complex than was assumed to be possible in the absence of a primary visual 
cortex. This coined a new research tradition into what is now referred to as affective blindsight, an approach that was extended to also include cross-modal processes by using not only unseen emotional stimuli but also auditory stimuli (de Gelder, Pourtois, \& Weiskrantz, 2002). Furthermore, some studies have demonstrated similar affective blindsight when using emotional body stimuli (de Gelder, 2006) rather than just faces. 


\section{GOALS AND AIMS OF THE PRESENT MANUSCRIPT}

The main goal of this thesis was to investigate the basic-level processing of objects and faces, and to infer knowledge about their underlying neural substrates by means of psychophysics setups. This goal can be subdivided into four specific aims. The first aim was to look at the explicit categorical boundaries between emotional faces and a non-face object category. In Chapter 2, I explored this question by investigating the presence of the information related to the emotional expression in an ambiguous visual stimulus. The ambiguous stimuli were generated by morphing between an emotional face and an object category. The results revealed that emotionrelated information is still seen as present in the ambiguous morphs, explicitly categorized as a non-face.

The second aim was to investigate whether the most basic level categorization of a stimulus as a face (face detection) or as a non-face (object detection) can be influenced by preceding stimuli (adaptation effect). For this I proposed a novel paradigm - Attribute-Selective Adaptation (ASA) - using rapid presentation of hundreds of stimuli from the same or different category of the test stimulus. In Chapter 3, I measured the detection thresholds of the test stimuli by adding spatial frequency noise to the original object with and without ASA. The results showed that the high-level within-category adaptation does deteriorate the participants' performance in basic level categorization.

The third aim was to investigate the extent of unconscious recognition of facial expressions. I was also interested in examining the relationship between the cognitive and metacognitive performances of face detection and emotion discrimination. To that aim, I employed a novel paradigm to render the face stimuli invisible by using the saccadic suppression effect. In Chapter 4, I presented the stimuli during saccadic eye movement and showed that the visibility of complex stimuli such as faces can drop out to the level of complete invisibility in most (>90\%) of the trials. In Chapter 5, using the same paradigm, I showed that although the face detection performance might drop to chance level, emotion categorization may still remain significant. This is probably the most important finding of the thesis as it is the first evidence showing that emotion perception can occur even in the absence of basic level detection of the face. Furthermore, the finding is in contradiction to most of the hierarchical models of face perception, which require a core representation of the face as the entry level for perception of emotions. Instead, it supports models in which 
separate neural processing is devoted to coarse face detection and emotional processing on the one hand, and recognition/identification on the other hand.

The fourth goal of this dissertation was to address some aspects of unconscious perception in the field of blindsight. In Chapter 6, I looked more closely at the audiovisual association learning abilities in a human patient with the rare condition of a bilateral cortical lesion of V1. I showed that audiovisual learning can take place in the absence of the primary visual cortex. Furthermore, I demonstrated that this effect is mediated by the superior colliculus. Chapter 7 addressed technical difficulties for the analysis of nearthreshold perception (including blindsight), with advanced statistical methods. I believe that this is an important platform for research on the unconscious. I also discussed the recent findings by Scott et al. (Scott, Dienes, Barrett, Bor, \& Seth, 2014) on the blind insight phenomenon and showed the statistical limitation of their approach to such findings with the use of their original dataset.

Finally, in Chapter 8, I presented my concluding remarks and discussed the main findings of the studies presented in this thesis in the broader context of the literature on emotional face perception. I commented on the technical and statistical difficulties of research on the unconscious, and indicate future directions and applications of this research. 


\section{References $^{1}$}

Afraz, S. R., \& Cavanagh, P. (2008). Retinotopy of the face aftereffect. Vision Res, 48(1), 42-54. doi: 10.1016/j.visres.2007.10.028

Atkinson, J., Anker, S., Braddick, O., Nokes, L., Mason, A., \& Braddick, F. (2001). Visual and visuospatial development in young children with Williams syndrome. Dev Med Child Neurol, 43(5), 330-337.

Bar, M., Kassam, K. S., Ghuman, A. S., Boshyan, J., Schmid, A. M., Dale, A. M., .. . Halgren, E. (2006). Top-down facilitation of visual recognition. Proceedings of the National Academy of Sciences of the United States of America, 103(2), 449-454. doi: 10.1073/pnas.0507062103²

Biederman, Irving. (1987). Recognition-by-components: a theory of human image understanding. Psychological review, 94(2), 115.

Bruce, C., Desimone, R., \& Gross, C. G. (1981). Visual properties of neurons in a polysensory area in superior temporal sulcus of the macaque. $J$ Neurophysiol, 46(2), 369-384.

Bruce, V., \& Young, A. (1986). Understanding face recognition. Br J Psychol, 77 ( Pt 3), 305-327.

Bruce, V., \& Young, A. W. (1986). Understanding face recognition. British journal of Psychology, 77, 305-327.

Calder, Andrew J., \& Young, Andrew W. (2005). Understanding the recognition of facial identity and facial expression. Nature reviews. Neuroscience, 6(8), 641-651.

Carey, Susan, De Schonen, S, \& Ellis, HD. (1992). Becoming a Face Expert [and Discussion]. Philosophical Transactions of the Royal Society B: Biological Sciences, 335(1273), 95-103.

Cheesman, J., \& Merikle, P. M. (1984). Priming with and without awareness. Perception \& Psychophysics, 36(4), 387-395.

Clarke, S., \& Miklossy, J. (1990). Occipital cortex in man: organization of callosal connections, related myelo- and cytoarchitecture, and putative boundaries of functional visual areas. J Comp Neurol, 298(2), 188-214.

\footnotetext{
${ }^{1}$ Referencing in each chapter is based on American Psychological Science (APA) Style, $6{ }^{\text {th }}$ Edition.

${ }^{2}$ According to APA, $6^{\text {th }}$ edition, if 7 or more authors contribute to one publication, the middle ones in the order need to be substituted by three dots in the reference list.
} 
Costen, Nicholas P, Parker, Denis M, \& Craw, lan. (1996). Effects of high-pass and low-pass spatial filtering on face identification. Perception \& Psychophysics, 58(4), 602-612.

de Gelder, B. (2006). Towards the neurobiology of emotional body language. Nature Reviews Neuroscience, 7(3), 242-249.

de Gelder, B., Frissen, I., Barton, J., \& Hadjikhani, N. (2003). A modulatory role for facial expressions in prosopagnosia. Proceedings of the National Academy of Sciences of the United States of America, 100(22), 1310513110. doi: 10.1073/pnas. 1735530100

de Gelder, B., Pourtois, G., \& Weiskrantz, L. (2002). Fear recognition in the voice is modulated by unconsciously recognized facial expressions but not by unconsciously recognized affective pictures. Proceedings of the National Academy of Sciences of the United States of America, 99(6), 4121-4126.

de Gelder, B., \& Rouw, R. (2000). Paradoxical configuration effects for faces and objects in prosopagnosia. Neuropsychologia, 38(9), 1271-1279.

de Gelder, B., \& Rouw, R. (2001). Beyond localisation: a dynamical dual route account of face recognition. Acta Psychol (Amst), 107(1-3), 183-207.

de Gelder, B., Vroomen, J., Pourtois, G., \& Weiskrantz, L. (1999). Non-conscious recognition of affect in the absence of striate cortex. Neuroreport, 10(18), 3759-3763.

Desimone, R., Albright, T. D., Gross, C. G., \& Bruce, C. (1984). Stimulus-selective properties of inferior temporal neurons in the macaque. $J$ Neurosci, 4(8), 2051-2062.

Desimone, R., \& Gross, C. G. (1979). Visual areas in the temporal cortex of the macaque. Brain research, 178(2-3), 363-380.

Doty, Robert W. (1971). Survival of pattern vision after removal of striate cortex in the adult cat. The Journal of Comparative Neurology, 143(3), 341-369. doi: $10.1002 /$ cne.901430306

Farah, M. J., Wilson, K. D., Drain, H. M., \& Tanaka, J. R. (1995). The inverted face inversion effect in prosopagnosia: evidence for mandatory, facespecific perceptual mechanisms. Vision Res, 35(14), 2089-2093. 
Felleman, D.J., \& Van Essen, D.C. (1991). Distributed hierarchical processing in primate cerebral cortex. Cerebral cortex, 1, 1-47.

Freud, Sigmund. (1922). The unconscious. The Journal of Nervous and Mental Disease, 56(3), 291-294.

Gattass, R, Gross, CG, \& Sandell, JH. (1981). Visual topography of V2 in the macaque. Journal of Comparative Neurology, 201(4), 519-539.

Gattass, R., Sousa, A. P., \& Gross, C. G. (1988). Visuotopic organization and extent of $V_{3}$ and $V_{4}$ of the macaque. $J$ Neurosci, 8(6), 1831-1845.

Gauthier, I., Behrmann, M., \& Tarr, M. J. (1999). Can face recognition really be dissociated from object recognition? J Cogn Neurosci, 11, 349-370.

Gauthier, I., Skudlarski, P., Gore, J. C., \& Anderson, A. W. (2000). Expertise for cars and birds recruits brain areas involved in face recognition. Nature neuroscience, 3(2), 191-197.

Gauthier, I., Tarr, M. J., Anderson, A. W., Skudlarski, P., \& Gore, J. C. (1999). Activation of the middle fusiform 'face area' increases with expertise in recognizing novel objects. Nature neuroscience, 2(6), 568-573.

Goffaux, V., Peters, J., Haubrechts, J., Schiltz, C., Jansma, B., \& Goebel, R. (2011). From coarse to fine? Spatial and temporal dynamics of cortical face processing. Cereb Cortex, 21(2), 467-476.

Green, D.M., \& Swets, J.A. (1966). Signal detection theory and psychophysics. New York: Wiley.

Gross, C. G., Bender, D. B., \& Rocha-Miranda, C. E. (1969). Visual receptive fields of neurons in inferotemporal cortex of the monkey. Science, 166(910), 1303-1306.

Gross, C. G., Rocha-Miranda, C. E., \& Bender, D. B. (1972). Visual properties of neurons in inferotemporal cortex of the Macaque. J Neurophysiol, 35(1), 96-111.

Haxby, J. V., Gobbini, M. I., Furey, M. L., Ishai, A., Schouten, J. L., \& Pietrini, P. (2001). Distributed and overlapping representations of faces and objects in ventral temporal cortex. Science, 293(5539), 2425-2430. 
Haxby, J. V., Hoffman, E. A., \& Gobbini, M. I. (2000). The distributed human neural system for face perception. Trends in cognitive sciences, 4(6), 223-233.

Horn, Gabriel. (2004). Pathways of the past: the imprint of memory. Nature Reviews Neuroscience, 5(2), 108-120.

Hubel, D. H., \& Wiesel, T. N. (1962). Receptive fields, binocular interaction and functional architecture in the cat's visual cortex. J Physiol, 160, 106-154.

Humphrey, Nicholas K, \& Weiskrantz, L. (1967). Vision in monkeys after removal of the striate cortex. Nature, 215(5101), 595-597.

Humphreys, G. W., \& Price, C. J. (2001). Cognitive neuropsychology and functional brain imaging: implications for functional and anatomical models of cognition. Acta Psychol (Amst), 107(1-3), 119-153.

Ishai, A., Ungerleider, L. G., \& Haxby, J. V. (2000). Distributed neural systems for the generation of visual images. Neuron, 28(3), 979-990.

Ishai, A., Ungerleider, L. G., Martin, A., Schouten, J. L., \& Haxby, J. V. (1999). Distributed representation of objects in the human ventral visual pathway. Proceedings of the National Academy of Sciences of the United States of America, 96(16), 9379-9384.

James, William. (1884). II.-What is an emotion? Mind(34), 188-205.

Johnson, M. H. (2005). Subcortical face processing. Nature reviews. Neuroscience, 6(10), 766-774. doi: 10.1038/nrn1766

Johnson, M. H., Dziurawiec, S., Ellis, H., \& Morton, J. (1991). Newborns' preferential tracking of face-like stimuli and its subsequent decline. Cognition, 40(1-2), 1-19.

Kemp, Richard, McManus, Chris, \& Pigott, Tara. (1990). Sensitivity to the displacement of facial features in negative and inverted images. Perception, 19(4), 531-543.

King, J., \& Dehaene, S. (2014). A model of subjective report and objective discrimination as categorical decisions in a vast representational space. Philosophical Transactions of the Royal Society B: Biological Sciences, 369(1641), 20130204. 
Kourtzi, Z., Betts, L. R., Sarkheil, P., \& Welchman, A. E. (2005). Distributed neural plasticity for shape learning in the human visual cortex. PLoS Biol., 3(7), e204.

Kourtzi, Z., \& Kanwisher, N. (2000). Cortical regions involved in perceiving object shape. J Neurosci, 20(9), 3310-3318.

Kovacs, G., Zimmer, M., Harza, I., Antal, A., \& Vidnyanszky, Z. (2005). Positionspecificity of facial adaptation. Neuroreport, 16(17), 1945-1949.

Lagercrantz, Hugo, Hanson, Mark A, Ment, Laura R, \& Peebles, Donald M. (2010). The newborn brain: neuroscience and clinical applications: Cambridge University Press.

Lueschow, A., Miller, E. K., \& Desimone, R. (1994). Inferior temporal mechanisms for invariant object recognition. Cereb Cortex, 4(5), 523531.

Macmillan, Neil A, \& Creelman, C Douglas. (2004). Detection theory: A user's guide: Psychology press.

Marr, David, \& Nishihara, Herbert Keith. (1978). Representation and recognition of the spatial organization of three-dimensional shapes. Proceedings of the Royal Society of London B: Biological Sciences, 200(1140), 269-294.

Martin, A., Wiggs, C. L., Ungerleider, L. G., \& Haxby, J. V. (1996). Neural correlates of category-specific knowledge. Nature, 379(6566), 649-652.

Marzi, CA, Tassinari, G, Aglioti, S, \& Lutzemberger, L. (1986). Spatial summation across the vertical meridian in hemianopics: A test of blindsight. Neuropsychologia, 24(6), 749-758.

MCCARTHY, ROSALEEN A, \& Warrington, Elizabeth K. (1986). Visual associative agnosia: a clinico-anatomical study of a single case. Journal of Neurology, Neurosurgery \& Psychiatry, 49(11), 1233-1240.

Mishkin, M., Ungerleider, L.G., \& Macko, K.A. (1983). Object vision and spatial vision: two cortical pathways. Trends Neurosci., 6, 414-417.

Monahan, Jennifer L, Murphy, Sheila T, \& Zajonc, Robert B. (2000). Subliminal mere exposure: Specific, general, and diffuse effects. Psychological science, 11(6), 462-466. 
Morris, J. S., DeGelder, B., Weiskrantz, L., \& Dolan, R. J. (2001). Differential extrageniculostriate and amygdala responses to presentation of emotional faces in a cortically blind field. Brain, 124(Pt 6), 1241-1252.

Moscovitch, M., Winocur, G., \& Behrmann, M. (1997). What is special about face recognition? Nineteen experiments on a person with visual object agnosia and dyslexia but normal face recognition. J Cogn Neurosci, 9, 555-604.

Näsänen, Risto. (1999). Spatial frequency bandwidth used in the recognition of facial images. Vision research, 39(23), 3824-3833.

Öhman, A. (2005). The role of the amygdala in human fear: Automatic detection of threat. Psychoneuroendocrinology, 30(10), 953-958.

Öhman, Arne, Flykt, Anders, \& Lundqvist, Daniel. (2000). Unconscious emotion: Evolutionary perspectives, psychophysiological data and neuropsychological mechanisms. In R. D. R. Lane, L. Nadel, \& G. L. Ahern (Eds.), Cognitive Neuroscience of Emotion. Series in Affective Science (pp. 296-327): Oxford University Press.

Owsley, C, Sekuler, R, \& Boldt, C. (1981). Aging and low-contrast vision: face perception. Investigative Ophthalmology \& Visual Science, 21(2), 362365 .

Perrett, D. I., Rolls, E. T., \& Caan, W. (1982). Visual neurones responsive to faces in the monkey temporal cortex. Exp Brain Res, 47(3), 329-342.

Pöppel, Ernst, Held, Richard, \& Frost, Douglas. (1973). Residual visual function after brain wounds involving the central visual pathways in man.

Potter, Mary C. (1976). Short-term conceptual memory for pictures. Journal of experimental psychology: human learning and memory, 2(5), 509.

Purcell, Dean G, \& Stewart, Alan L. (1986). The face-detection effect. Bulletin of the Psychonomic Society, 24(2), 118-120.

Purcell, Dean G, \& Stewart, Alan L. (1988). The face-detection effect: Configuration enhances detection. Perception \& Psychophysics, 43(4), 355-366. 
Rafal, R., Smith, J., Krantz, J., Cohen, A., \& Brennan, C. (1990). Extrageniculate vision in hemianopic humans: saccade inhibition by signals in the blind field. Science, 250(4977), 118-121.

Scott, Ryan B, Dienes, Zoltan, Barrett, Adam B, Bor, Daniel, \& Seth, Anil K. (2014). Blind Insight Metacognitive Discrimination Despite Chance Task Performance. Psychological science, 0956797614553944.

Sergent, J., Ohta, S., \& MacDonald, B. (1992). Functional neuroanatomy of face and object processing. A positron emission tomography study. Brain, 115(Pt 1), 15-36.

Sewards, T. V., \& Sewards, M. A. (2002). Innate visual object recognition in vertebrates: some proposed pathways and mechanisms. Comp Biochem Physiol A Mol Integr Physiol, 132(4), 861-891.

Sidis, Boris. (1898). The psychology of suggestion: D. Appleton \& Company.

Sprague, James M., De Weerd, Peter, Xiao, Deng-Ke, Vandenbussche, Erik, \& Orban, Guy A. (1996). Orientation discrimination in the cat: Its cortical locus II. Extrastriate cortical areas. The Journal of Comparative Neurology, 364(1), 32-50. doi: 10.1002/(sici)10969861(19960101)364:1<32::aid-cne4>3.0.c0;2-t

Tanaka, J. W. (2001). The entry point of face recognition: evidence for face expertise. J Exp Psychol Gen, 130(3), 534-543.

Tanaka, K. (1993). Neuronal mechanisms of object recognition. Science, 262(5134), 685-688.

Tanaka, K. (1996). Representation of Visual Features of Objects in the Inferotemporal Cortex. Neural Netw, 9(8), 1459-1475.

Webster, M. J., Bachevalier, J., \& Ungerleider, L. G. (1994). Connections of inferior temporal areas TEO and TE with parietal and frontal cortex in macaque monkeys. Cerebral cortex, 4(5), 470-483.

Tsao, D. Y., Freiwald, W. A., Tootell, R. B., \& Livingstone, M. S. (2006). A cortical region consisting entirely of face-selective cells. Science, 311(5761), 670674 . 
Tsao, D. Y., Schweers, N., Moeller, S., \& Freiwald, W. A. (2008). Patches of faceselective cortex in the macaque frontal lobe. Nature neuroscience, $11(8), 877-879$.

Weiskrantz, L. (1986). Blindsight : a case study and implications. Oxford: Clarendon Press.

Weiskrantz, L., Cowey, A., \& Le Mare, C. (1998). Learning from the pupil: a spatial visual channel in the absence of $V_{1}$ in monkey and human. Brain, 121 (Pt 6), 1065-1072.

Weiskrantz, L., Warrington, E. K., Sanders, M.D., \& Marshall, J. (1974). Visual capacity in the hemianopic field following a restricted occipital ablation. Brain, 97, 709-728.

Yin, Robert K. (1969). Looking at upside-down faces. Journal of Experimental Psychology, 81(1), 141. 


CHAPTER 2

\title{
SEEING EMOTIONS IN THE \\ OBJECTS
}

\author{
Emotion Categorization does not Depend on Explicit Face \\ Categorization
}

Based on the article:

Mehrdad Seirafi, Peter De Weerd, and Beatrice de Gelder. "Emotion categorization does not depend on explicit face categorization." Journal of vision 13.2 (2013): 12. 


\section{ABSTRACT}

Face perception and emotion recognition have been extensively studied in the past decade; however, the relation between them is still poorly understood. A traditional view is that successful emotional categorization requires categorization of the stimulus as a 'face', at least at the basic level. Here we tested whether without explicit categorization of a stimulus as a face emotional information could still be recognized accurately. For this purpose we created a stimulus set in which facial stimuli expressing a range of happy-to-fear emotions were morphed into another object category (shoe). Interestingly, participants categorized emotions with great accuracy in stimuli that contained so little face information that they were explicitly categorized as shoes. Hence, our results show that accurate emotion categorization can take place in stimuli that contain surprisingly little face information. This finding raises interesting questions about the extent to which processes leading to emotion recognition and categorical face perception might be separable.

Keywords: detection, discrimination, face recognition, object recognition, visual cognition 


\section{1) Introduction}

Object recognition has been classically viewed to occur in a series of sequential processing stages: detection, categorization and identification. At the first stage (object detection), the overall shape of the object is segregated from its background (Driver \& Baylis, 1996). Then, the detected object is categorized in supra- and sub-ordinate levels (Nakayama, He, \& Shimojo, 1995). Eventually, the object is identified at the final level of object recognition. However, this sequential view on object processing has been challenged in several studies. For example, Peterson and Gibson (Peterson \& Gibson, 1994) showed that object category can influence the performance at detection level, in stimulus conditions that prevented perceptual categorization, thus arguing against the unidirectional view that detection precedes further categorical processing. Moreover, Grill-Spector and Kanwisher (Grill-Spector \& Kanwisher, 2005) proposed that detection and basic-level categorization take place almost at the same stage. This is a challenge to the sequential processing idea as well, because here it is assumed that an object, once detected, may be categorized without additional processing (Bar, 2004; Bar et al., 2001).

A similar debate is present in the literature on face perception. Traditional models of face recognition assume a sequential series of processing steps, including figure-ground segregation and categorization processes yielding a structural description that once achieved is the basis of two further, separate routes of face processing: (1) person identification and (2) recognition of emotional expression (Bruce \& Young, 1986; Morris, de Gelder, Weiskrantz, \& Dolan, 2001). One debate in the face processing literature is about the extent to which the processes involved in face- and emotion processing interact (Calder \& Young, 2005). Another more fundamental debate is whether the two processes of face (body) identification and emotion recognition make use of the same initial structural representation of the face (body). There is rising evidence that emotion categorization uses a coarse face representation that is not sufficient for, or altogether different from face representations involved in face categorization and identification (de Gelder \& Rouw, 2001; de Gelder, Snyder, Greve, Gerard, \& Hadjikhani, 2004; Garrido, Barnes, Sahani, \& Dolan, 2012; Gschwind, Pourtois, Schwartz, Van De Ville, \& Vuilleumier, 2012; Johnson, 2005). If so, it should be possible to categorize correctly the emotional expression of a stimulus that contains a face of which the representation is too coarse to be categorized as a face. To evaluate this hypothesis, we tested whether in stimuli containing increasingly degraded structural face information, 
accurate categorization of the facially expressed emotion was always associated with an explicit categorization of the stimulus as a face. To that aim, we devised a stimulus set based on a two way morph procedure involving two object categories and two emotional facial expressions. Specifically, a series of face images from the same identity was morphed from one to another emotional expression, and each image of the series was then morphed to the same exemplar of another object category (shoes). Using this stimulus set, we tested whether emotion categorization is possible at morph levels in which structural face information is degraded to a level that leads to explicit categorization of the stimuli as a shoe rather than a face. Three possible outcomes were envisaged for this experiment: First, it is possible that a high level of face-related information is required to permit accurate emotion categorization. This means that accurate emotion categorization would be possible only for face-object morphs that are almost always explicitly categorized as faces. Second, it is possible that the physical information required to categorize the intermediate morphs as faces in most of the trials is sufficient to permit emotion categorization. Third, emotion categorization may be possible at face-object morph levels that are explicitly categorized as shoes and that give insufficient information for the stimuli to be categorized as a face.

\section{1) General Methods}

\section{Stimuli}

Original face images were color images of two males (KDEF identities: AM10 and AM14), each displaying two different emotions of happy and fearful (2 identities $\times 2$ emotions) selected from a sub-set of Karolinska Directed Emotional Faces (KDEF) (Lundqvist, Flykt, \& Öhman, 1998), previously validated to be correctly $(>90 \%)$ categorized as happy and fearful in a pilot study on 40 participants. The shoe stimulus was selected from our own shoe database (de Gelder, Bachoud-Levi, \& Degos, 1998).

The stimuli were preprocessed as follows: First, all the original images were cropped and resized to fit into an oval-like template-between face and shoe outline- by Adobe Photoshop CS4 (Adobe; http://adobe.com). Then, all the average pixel values for each image were shifted to 128 in Matlab 2010a (Mathworks; http://www.mathworks.com). Afterwards, all the pictures were 
overlaid on top of a gray noisy background with the same average luminance of 128.

The preprocessed images were then submitted to the morphing software, FantaMorph 4.2.6 (Abrosoft; http://abrosoft.com), to generate the morphs between two emotional faces and the shoe. To that aim, we first morphed the two emotional faces from the same identity to each other in 6 equal morphing steps, resulting in 7 stimuli from very happy to very fearful faces. Then, each of the 7 emotional face stimuli was morphed to a single shoe stimulus in 12 morphing steps.

We used two different subsets of the morphed stimuli in the two experiments: (a) The odd number face-shoe morph levels (1, 3, 5, 7, 9, 11 and 13) in Experiment 1 corresponded to face content percentiles of 100, 83, 66, 50, 33, 17,0 ; (b) the second half of the face-shoe morph levels (7, 8, 9, 10, 11, 12 and 13) in Experiment 2 corresponded to face content percentiles of 50, 42, 32, 25, 17, 8, 0 . Note that axis labels in Fig 2 and 3 are ordinal values, of which the \% face content is defined by above specified labels.

Each face-shoe morph level subset -except the shoe anchor-point consisted of 7 levels of happy-fear morphed stimuli. As a result, a total of 43 stimuli ranging in two dimensions of emotion (fearful face, happy face), and object category (face-shoe) were tested in each experiment (Figure 1).

The visual stimuli were presented on a 17-inch Samsung LCD monitor with a preset refresh rate of $60 \mathrm{~Hz}$, using a Pentium Core 2 Duo, $3.0 \mathrm{GHz}$ computer using E-prime 2.0 (Psychology Software Tools Inc., Pittsburgh, USA; www.pstnet.com). The subjects were sitting at a distance of approximately 65 $\mathrm{cm}$ from the monitor in a dimly light room.

\section{Data Analysis}

In each task, the proportion response for each corresponding morph-level was calculated and all the task-relevant psychometric functions were fitted. Furthermore, statistical analysis on the thresholds was performed by SPSS (PASW Statistics 18.0; http://www.spss.com/). 


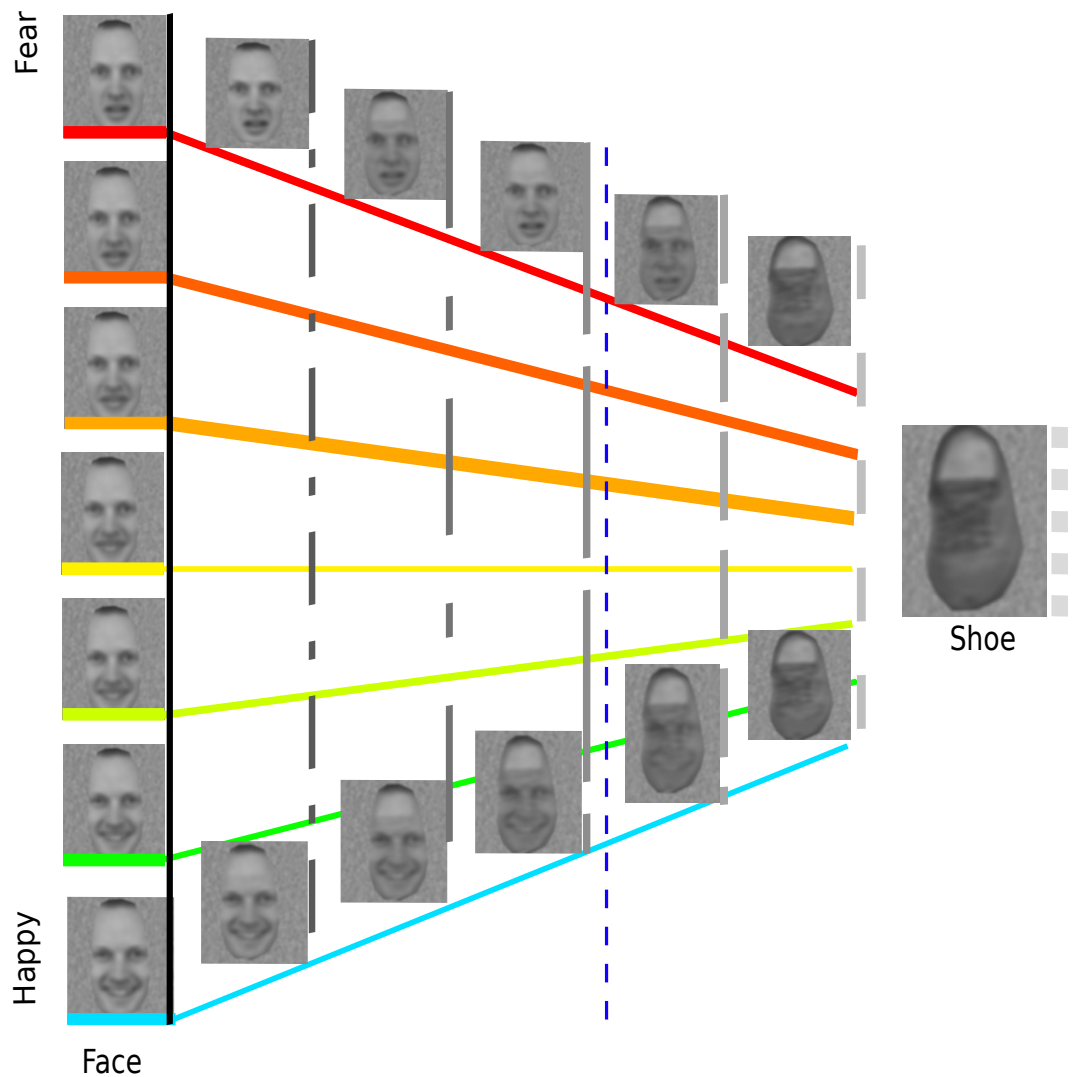

Figure 1 Samples of morphed stimuli, with colored lines representing faces (KDEF identity: AM10) along the fear (red, morph 1, 100\% fear) to happy (cyan, morph 7, 0\% fear) emotion dimension, and grey level lines representing stimuli along the face (black, morph 1, 100\% face) to shoe (light grey fine dashed line, morph 7, $0 \%$ face) object dimension. The dotted blue line represents a hypothetical perceptual boundary between the face and the shoe categories.

Psychometric fitting. The Psychometric functions were obtained by using local linear fitting (McCullagh \& Nelder, 1989; Zychaluk \& Foster, 2009). The advantage of this method is that it is model-free; it works best when the exact shape of the underlying psychometric function is uncertain. In this method, the only assumption about the shape of the psychometric function is its smoothness. By a Taylor expansion, any smooth function can be approximated locally by a linear function:

$I\left(P\left(x_{i}\right)\right)=\eta \approx a_{0}+a_{1}\left(x_{i}-x\right)$, 
where the parameters $a_{0}, \alpha_{1}$ depend on $x$ and their values are estimated by maximizing local log-likelihood,

$I(x)=\sum_{i}\left[k_{i} \ln \left(P\left(x_{i}\right)\right)+\left(m_{i}-k_{i}\right) \ln \left(1-P\left(x_{i}\right)\right)\right] w\left(x, x_{i}\right)$.

The weights $w\left(x, x_{i}\right)$ determine the influence of each point $x i$ on the estimate at a point $x$, so that the further the points $x$ and $x_{i}$ are apart, the smaller the value of $w\left(x, x_{i}\right)$.

To decide the optimal bandwidth for each condition, we first estimated the appropriate loss function, which measures the discrepancy between the true psychometric function and the estimate. Next, we used cross-validated deviance to find the optimal bandwidth. Then, $1000 \mathrm{x}$-values were generated and their corresponding fit were estimated by locglmfit function in model-free toolbox for Matlab (Zychaluk \& Foster, 2009). Finally, the threshold and slope of the fit were estimated by 200 bootstrap iterations.

\section{Experiment 1: Emotion categorization versus face/object categorization}

\section{Methods}

Participants. We recruited 10 students (8 female, $M_{\text {age: }}$ 23, age range: 18-27) from Maastricht University through local advertisements for this study. The study was conducted according to local ethics committee. The subjects gave their informed consent to participate in the experiment for either 1 course credit or 7.5 Euro. All participants were right-handed, with normal or correctedto-normal vision and naive to the purpose of the experiment. One subject was removed from the analysis because of very strong (>90\%) preference towards face response for all the stimuli including the shoe anchor-point.

Procedure. The participants performed two different tasks (face-shoe categorization and emotion categorization) one after the other in 4 experimental blocks (counterbalanced among the subjects). In the face-shoe categorization task, the participants reported whether they perceived the stimuli as a face or a shoe (face versus object). In the emotion categorization task, the participants had to report the emotional content (happy versus fear) of the presented stimulus even if not perceiving the stimulus as face. Left and 
right button presses corresponded respectively to face and shoe responses in the face-shoe categorization task and to fear and happy responses in the emotion categorization task.

One block consisted of 8 repetitions of each trial in which one of the 43 images was shown for $66 \mathrm{~ms}$ and in which the participants performed the instructed task ( $8 \times 43$ trials for a single block). One trial proceeded as follows: the fixation cross was presented in the center of screen for $500 \mathrm{~ms}$, followed by a stimulus for $66 \mathrm{~ms}$. Then a blank grey screen was shown and the participants were supposed to perform the respective task of the block. Trials were presented in a pseudo-random order, such that in each subsequent group of 43 trials each stimulus was presented once. Within each group, stimulus order was random.

Prior to each block, the instructions were presented on the screen and the subjects were trained for 2 minutes to learn the button associations and get familiar with the stimuli. When training was over, the instructions were displayed again and the experimental block started with the subjects' button press.

\section{Results and Discussion}

Participants performed an object categorization (face or shoe) and an emotion categorization task (happy or fear) for each stimulus in separate experimental blocks. We first averaged the response to each face-shoe morph for different emotion (happy-fear morph) levels in all subjects. Then, we fitted nonparametric psychometric curves to the percentage shoe responses as a function of face-shoe level and calculated the 50\% threshold of each participant in the face-shoe categorization task. We used repeated measures ANOVA to compare the differences in face-shoe categorization thresholds among different emotion levels, with emotion as the within-subject factor and participants as the between-subject factor. We found that emotion level did not influence object categorization thresholds $(F(6,48)=0.39, p>0.1$; sphericity assumed $)$. This conclusion based on analysis of thresholds is corroborated by the average psychometric curves seen in Figure 2A, showing complete overlap across different emotion levels.

More importantly, we also found that emotion categorization was strikingly robust against degradation of face information (Fig $2 B$ ). The results show that for morph levels containing as little as $17 \%$ of face information (corresponding to face-shoe morph 6), emotion categorization remained 
possible. This is supported by the goodness of fit in the $17 \%$ face condition, which averaged over participants was $86 \%(S D=8.5 \%$ ).

Note that there is an overall bias to categorize stimuli as shoes rather than faces (Fig 2A). This is likely due to the choice of a shoe stimulus with anchor points for the morphing procedure that matched well to a face.

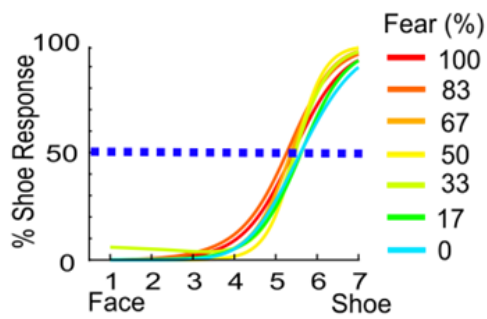

Morph Level
B

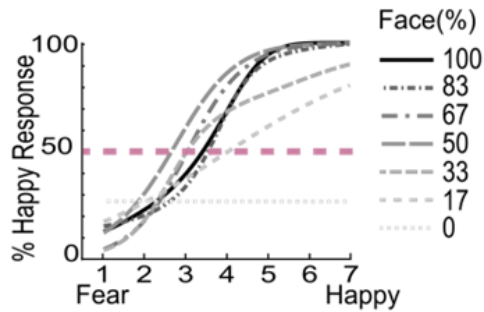

Morph Level

Figure 2 (A) Face-Shoe categorization responses as function of face-shoe morph level averaged over all subjects; (B) Fear-Happy categorization responses as a function of different levels of Face-Shoe morphing. Grey-level/line-style coding of plots for different face-shoe morph levels corresponds to grey-level coding as in Figure 1 . The morph levels on $x$-axis represent the ordinal sequence of the morphs described in General Methods section.

The results from this experiment suggest that successful emotion categorization may occur for ambiguous stimuli that are perceived as a shoe in most trials. However, the object categorization task and the emotional expression categorization tasks were performed separately, in different blocks of trials. We therefore cannot exclude that although the stimuli containing only $17 \%$ facial information were categorized as shoes in the majority of trials, the correct emotion categorization performance may reflect a minority of trials in which the participants would classify the stimuli as faces if they had been asked (while performing emotion categorization at chance level for the rest of the trials). Alternatively, as we would like to argue, participants may use the $17 \%$ face information in the stimuli to categorize emotion correctly without explicitly categorizing the stimulus as a face and, instead, categorizing it as a shoe.

Note that the results from the shoe anchor-point in the emotion categorization task showed a bias towards fearful emotion in all subjects (Fig $2 \mathrm{~B}$ ). This is likely related to the emotional valance of this specific category of objects (de Gelder, 2005). 


\section{3| Experiment 2: Dual response categorization task}

The results from Experiment 1 indicate that emotion categorization can still take place when the observers do not categorize the visual stimulus as a face in most of the trials. The strength of this conclusion, however, was limited by the fact that the object and emotion categorization tasks were done in different experimental blocks. Here, we aimed to strengthen this conclusion by testing whether emotion categorization would remain possible even on those trials in which the weak face information present in the stimulus did not lead to explicit categorization as a face. To test this hypothesis, we used a dual task design, in which participants performed both the object categorization and the emotion categorization task within each single trial. Moreover, based on the observation in Figure 2 showing complete face categorization for ordinal morph levels 1-4 (i.e., $0 \%$ shoe responses), we shifted the range of object morph levels towards levels containing less face information (see General Methods). This allowed us to collect a sufficient number of trials resulting in a shoe categorization response, required to test the efficiency of emotion categorization in stimuli not categorized as a face.

\section{Methods}

Participants. Ten students (8 female, $M_{\text {age }}: 23$, age range: $18-27$ ) from Maastricht University were recruited through local advertisements for this study. The study was conducted after approval by the local ethics committee. The subjects gave their informed consent to participate in the experiment for either 1 course credit or 7.5 Euro. All participants were right-handed, with normal or corrected-to-normal vision and naive to the purpose of the experiment. Six participants had already participated in Experiment 1 two weeks prior to Experiment 2.

Procedure. The design for this experiment was similar to Experiment 1 using another subset of morphed stimuli (see General Methods section); the only difference was that the participants were asked to perform both tasks one after each other immediately after each stimulus. After each stimulus presentation, the subject decided both the Emotional content (task E) and the Object category (Task O) of the stimulus. The trials were divided in two blocks, one for each of the two possible task orders (OE or EO). Each participant did one block for each order, but the sequence of task order was counterbalanced 
over participants (i.e., half of the participants did EO in the first block and EO in the second; the other half did the converse). Task order in a block was cued by a verbal instruction appearing on the screen prior to the start of the block.

A single block consisted of 8 trials for each of the 43 images (344 trials). Trials were presented in a pseudo-random order, such that in each subsequent group of 43 trials each stimulus was presented once. Within each group, stimulus order was random. One trial proceeded as follows: the fixation cross was presented in the center of the screen for $500 \mathrm{~ms}$, followed by a stimulus for 66 ms. Then a blank grey screen was shown and the participants were supposed to perform both tasks one after the other according to the instructed order. Up and down arrow keys corresponded to the happy and fearful response in the emotion categorization task; left and right arrow keys corresponding to face and shoe response in the object categorization task, respectively. Participants were reminded on a trial by trial basis of the response requirements by showing the cue words happy and fear just above and below the fixation spot in the emotion categorization task. Similarly, they were reminded of the response requirements by cue words face and shoe to the left and right of the fixation spot in the object categorization task. Participants self-initiated the experiment with a button press. Prior to each block, participants were familiarized with the stimuli and button associations in the two tasks during a brief training period lasting 2 minutes.

\section{Results and Discussion}

To decide whether we could pool the data from all trials from all participants to test our main hypothesis, we first explored whether the order of tasks had any effect on the thresholds in the face-shoe categorization task. It is possible, for example, that on a given trial, having responded "shoe" (not face) in the first task might have affected the decision of the participant in the subsequent emotion task. We pooled the data for each task order over all participants, and compared the percentage happy responses as a function of the emotion morph level for the $8 \%$ and $17 \%$ face conditions. This resulted in two psychometric curves that were indistinguishable, as confirmed by ANOVA of \% happy responses, using as factors emotion level and task order $(F(1,8)=2.04, p>0.1)$. Furthermore, there was a rigid psychometric function underlying the emotion categorization response of the subjects with no effect of task order (thresholds for EO-OE orders were $3.73 \pm 0.18$ and $3.93 \pm 0.16$, respectively). 
Hence, we proceeded to pool the data from all subjects across task orders and studied the trials in which the participants categorized the stimuli as shoe, pooling data from the $8 \%$ and $17 \%$ face conditions. The shoe anchor-point ( $0 \%$ face) was not included. When plotting \% happy responses as a function of morph level in the combined $8 \%$ and $17 \%$ face conditions (Figure 3), we found a smooth function with a threshold of $3.8 \pm 0.4$. Furthermore, to verify the discriminating effect of underlying emotion, we compared the tails of the function with the $50 \%$ threshold. To that goal, we grouped the emotion morphs based on the perceived emotion categories (fear: 1, 2; happy: 6, 7) and applied one-sample t-test. The results show that there are significant differences for both groups below (fear: $t(127)=-12.1$ ) and above (happy: $t(119)=9.7)$ the threshold.

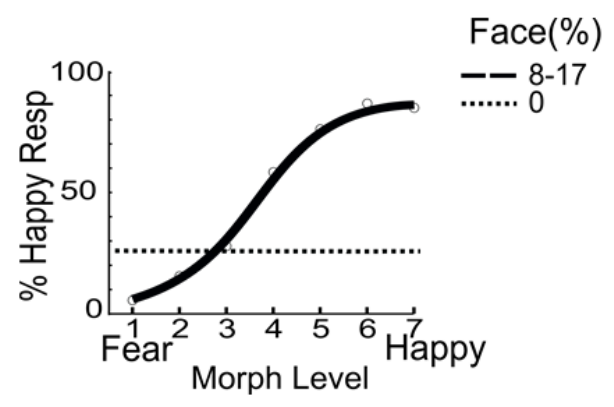

Figure 3 Emotion categorization as a function of face-shoe morph levels for trials in which the stimulus was categorized as a shoe.

Thus, Fig 3 shows that for the trials in which participants categorized stimuli that contained only $8 \%-17 \%$ face information as shoes, there was nevertheless robust categorization of emotions contained in the degraded and unperceived faces. These data are compelling because the successful emotion categorization was performed in trials in which participants categorized the stimuli as a shoe. Hence, the data from the dual task design in this experiment provide a confirmation and validation of results and conclusions from Experiment 1.

\section{4| General Discussion}

The present study shows that accurate emotion categorization can take place in the absence of explicit face-object categorization, and in the presence of very little residual face information. This raises interesting questions about the 
amount of face information necessary for emotion categorization, and about the way in which this residual face information is processed. In Experiment 1, we investigated emotion categorization performance for the stimuli in which the face-related information was not sufficient for categorization of the visual stimuli as face. The results showed that for intermediate face-to-shoe morphed stimuli that showed a strong average bias to be explicitly categorized as shoe, the participants could still reliably categorize the emotional content. In Experiment 2, we explored whether the high emotion categorization performance for the stimuli categorized on average as shoe in Experiment 1 was due to those trials in which the stimulus was categorized as a face. For this purpose we used a dual task with participants performing both emotion categorization and face-object categorization after each stimulus presentation. The results indicate that even in the trials in which the subjects were explicitly categorizing the stimuli as shoe, and in which the stimuli were selected to have very little physical face information, they could still reliably categorize the emotional content of the image.

This result is important because it fits with several robust findings that have been taken as evidence that different, even specialized, processes underlie face and object recognition (Tanaka \& Farah, 1993; Valentine, 1988; Yin, 1969). Some researchers have proposed that faces are special, possibly not sharing any or sharing very little processing routes with objects (Farah, Wilson, Drain, \& Tanaka, 1998; Grill-Spector, Knouf, \& Kanwisher, 2004). Furthermore, it has been previously demonstrated that subordinate categorization in the expertise domain of the viewer can be as accurate and as fast as the basic-level categorization (Rosch, Mervis, Gray, Johnson, \& Boyes-Braem, 1976). More specifically, Tanaka (Tanaka, 2001) showed that both basic-level and subordinate level categorizations take place with similar timing and accuracy. The present study suggests that the sub-ordinate categorization of emotions in faces can outperform the explicit basic level categorization between two object categories.

Several factors may contribute to performance in categorization tasks. Priming is one such factor, and visual face processing mechanisms have indeed been shown to be easily primed by verbal instructions and previously presented stimuli. For example, Bentin et al. (Bentin, Sagiv, Mecklinger, Friederici, \& von Cramon, 2002) showed that face-specific brain activity can be evoked by nonface objects after they had been seen embedded in schematic faces. If we consider the stimuli with high face content as priming stimuli for stimuli with low face content, this may also contribute to the successful emotional 
categorization of stimuli with very low face content. This in itself, however, does not invalidate our conclusions, as our data do suggest that the 'primed' face representations permit highly successful emotion categorization in stimuli that are explicitly categorized as shoes, and contain as little as $8 \%$ of the original face content. A related consideration is that the morphing span of the faceshoe stimuli may affect the category boundary, which is relevant in Experiment 2, where we worked with a small subset of the morphing range biased strongly towards stimuli with low face content. To counter a possible bias to categorize stimuli as shoes, we presented the whole range of stimuli in the practice phase. This may not have prevented fully a bias in participants to classify the stimuli as shoes, but such bias makes the finding of very accurate emotion categorization in these stimuli all the more compelling.

An important open question in the interpretation of our data is related to the 2-Alternative Forced Choice (2AFC) paradigm used in the object categorization task. Because of this, it cannot be excluded that participants, despite a classification of a stimulus as a shoe, were still aware of some human facial information in the shoe. The explicit categorization of a stimulus as a shoe does not fully exclude awareness of the facial information, and this might then drive the highly successful emotional categorization. However, the very brief stimulus presentation, as well as the very small amount of human face information that still drove the emotional categorization responses, and the mixing of the human face information with the face-like features of the (pure) shoe stimulus (chosen to make morphing with a human face feasible), are all factors that limit the likelihood of this explanation. Further experiments, using some additional measure of residual face perception or a type of confidence measure could address this issue, as well as the question whether residual face information guides perception in a conscious or an unconscious manner.

Because it cannot be fully excluded that physically present face information in stimuli categorized as shoes could still have been used in a conscious or unconscious manner to construct a basic representation of a face, it also cannot be fully excluded that a subordinate emotion categorization is derived from it, in line with more sequential models of face processing (see Introduction). In particular, our finding that accurate explicit fear-happy emotion categorization, but not face-shoe object categorization, survives a strong reduction in the amount of physical face-related information in the stimuli could be seen as supporting a sequential model in which a common face representation provides input to a highly sensitive mechanisms of emotion categorization and a less sensitive mechanism of object categorization. This 
appears in agreement with the well-known effects of affective salience on visual attention (Calvo \& Nummenmaa, 2008; Eimer \& Holmes, 2007; Palermo \& Rhodes, 2007). However, despite possible alternative theoretical explanations and open empirical questions, our data may also be seen as support for a possible separation between processing streams for face representations used for emotion categorization and face representations used for conscious object categorization. The present evidence gives interesting clues that provide new avenues for further tests of two-pathway theories of face perception.

Traditional models of face processing are built on the notion that processing involves two sequentially organized stages, one of category assignment and the other of affective valence attribution. The alternative view (developed more in the context of affective valence processing than object recognition per se) postulates two parallel pathways, one of which is devoted to rapid extraction of relevant information before detailed processing of the visual properties (Anderson, Christoff, Panitz, De Rosa, \& Gabrieli, 2003; Bar et al., 2001; de Gelder \& Rouw, 2001; Garrido et al., 2012; Rudrauf et al., 2008; Vuilleumier, 2005). In this alternative account, emotion categorization uses a coarse representation of faces that might not be sufficient for categorizing the visual object as a face like in the ambiguous morphs we used in our study, but that might still permit successful emotion categorization. The existence of different entry representations and possibly different types of information for different facial information attributes may be tested in future experiments by assessing the effect of structural facial transformations on face and object categorization. A better understanding of how our visual system extracts emotions from a face is theoretically important, and we therefore believe our findings can give useful input to both computational modelling efforts and neurophysiological investigations of emotion and face perception. Further research is needed to understand the information present in the stimuli that drives the categorization of emotion in stimuli that are not explicitly categorized as faces.

\section{5| Conclusion}

The present study provides the first evidence that the ability to categorize a stimulus as fearful or happy does not require that the participants explicitly categorize the stimulus as a face, and can take place in stimuli that contain very little physical face information. 


\section{Acknowledgements}

This study was partly supported by Human Frontiers Science Program RGP54/2004, NWO Nederlandse Organisatie voor Wetenschappelijk Onderzoek 400.04081, and EU FP6-NEST-COBOL043403 and FP7 TANGO to Beatrice de Gelder, and NWO VICI grant to Peter De Weerd. 


\section{References}

Anderson, A. K., Christoff, K., Panitz, D., De Rosa, E., \& Gabrieli, J. D. (2003). Neural correlates of the automatic processing of threat facial signals. $J$ Neurosci, 23(13), 5627-5633.

Bar, M. (2004). Visual objects in context. Nature reviews. Neuroscience, 5(8), 617629. doi: $10.1038 / \mathrm{nrn} 1476$

Bar, M., Tootell, R. B., Schacter, D. L., Greve, D. N., Fischl, B., Mendola, J. D., . . . Dale, A. M. (2001). Cortical mechanisms specific to explicit visual object recognition. Neuron, 29(2), 529-535.

Bentin, S., Sagiv, N., Mecklinger, A., Friederici, A., \& von Cramon, Y. D. (2002). Priming visual face-processing mechanisms: electrophysiological evidence. Psychol Sci, 13(2), 190-193.

Bruce, V., \& Young, A. (1986). Understanding face recognition. Br J Psychol, 77 ( Pt 3), 305-327.

Calder, Andrew J., \& Young, Andrew W. (2005). Understanding the recognition of facial identity and facial expression. Nature reviews. Neuroscience, 6(8), 641-651.

Calvo, M. G., \& Nummenmaa, L. (2008). Detection of emotional faces: salient physical features guide effective visual search. J Exp Psychol Gen, 137(3), 471-494. doi: 10.1037/ao012771

de Gelder, B. (2005). Non-conscious emotions: new findings and novel perspectives. In L. F. Barret, P. M. Niedenthal, \& P. Winkielman (Eds.), Emotion and Consciousness. (pp. 123-149). New York: The Guilford Press.

de Gelder, B., Bachoud-Levi, A. C., \& Degos, J. D. (1998). Inversion superiority in visual agnosia may be common to a variety of orientation polarised objects besides faces. Vision research, 38(18), 2855-2861.

de Gelder, B., \& Rouw, R. (2001). Beyond localisation: a dynamical dual route account of face recognition. Acta Psychol (Amst), 107(1-3), 183-207.

de Gelder, B., Snyder, J., Greve, D., Gerard, G., \& Hadjikhani, N. (2004). Fear fosters flight: A mechanism for fear contagion when perceiving 
emotion expressed by a whole body. Proceedings of the National Academy of Sciences U S A, 101(47), 16701-16706.

Driver, J., \& Baylis, G. C. (1996). Edge-assignment and figure-ground segmentation in short-term visual matching. Cognitive psychology, 31(3), 248-306.

Eimer, M., \& Holmes, A. (2007). Event-related brain potential correlates of emotional face processing. Neuropsychologia, 45(1), 15-31. doi: So0283932(06)00157-6

Farah, M. J., Wilson, K. D., Drain, M., \& Tanaka, J. N. (1998). What is "special" about face perception? Psychol Rev, 105(3), 482-498.

Garrido, M. I., Barnes, G. R., Sahani, M., \& Dolan, R. J. (2012). Functional evidence for a dual route to amygdala. Curr Biol, 22(2), 129-134. doi: Sog60-9822(11)01371-6

Grill-Spector, K., \& Kanwisher, N. (2005). Visual recognition: as soon as you know it is there, you know what it is. Psychol Sci, 16(2), 152-160.

Grill-Spector, K., Knouf, N., \& Kanwisher, N. (2004). The fusiform face area subserves face perception, not generic within-category identification. Nature neuroscience, 7(5), 555-562.

Gschwind, M., Pourtois, G., Schwartz, S., Van De Ville, D., \& Vuilleumier, P. (2012). White-Matter Connectivity between Face-Responsive Regions in the Human Brain. Cereb Cortex. doi: bhr226

Johnson, M. H. (2005). Subcortical face processing. Nature reviews. Neuroscience, 6(10), 766-774. doi: 10.1038/nrn1766

Lundqvist, D., Flykt, A., \& Öhman, A. (1998). The Karolinska Directed Emotional Faces-KDEF. CD-ROM from Department of Clinical Neuroscience, Psychology section, Karolinska Institutet, Stockholm, Sweden: ISBN 91630-7164-9.

McCullagh, P., \& Nelder, John A. (1989). Generalized linear models (2nd ed.). London; New York: Chapman and Hall. 
Morris, J. S., de Gelder, B., Weiskrantz, L., \& Dolan, R. J. (2001). Differential extrageniculostriate and amygdala responses to presentation of emotional faces in a cortically blind field. Brain, 124(Pt 6), 1241-1252.

Nakayama, K, He, Z.J., \& Shimojo, S. (1995). Visual surface representation: A critical link between lower-level and higher-level vision. In S. M. Kosslyn, D. N. Osherson, \& e. al. (Eds.), Visual cognition: An invitation to cognitive science (Vol. 2, pp. 1-70). Cambridge, MA, USA: MIT press.

Palermo, R., \& Rhodes, G. (2007). Are you always on my mind? A review of how face perception and attention interact. Neuropsychologia, 45(1), 75-92. doi: So028-3932(06)00165-5

Peterson, M. A., \& Gibson, B. S. (1994). Object recognition contributions to figure-ground organization: operations on outlines and subjective contours. Percept Psychophys, 56(5), 551-564.

Rosch, Eleanor, Mervis, Carolyn B., Gray, Wayne D., Johnson, David M., \& BoyesBraem, Penny. (1976). Basic objects in natural categories. Cognitive psychology, 8(3), 382-439.

Rudrauf, D., David, O., Lachaux, J. P., Kovach, C. K., Martinerie, J., Renault, B., \& Damasio, A. (2008). Rapid interactions between the ventral visual stream and emotion-related structures rely on a two-pathway architecture. J Neurosci, 28(11), 2793-2803.

Tanaka, J. W. (2001). The entry point of face recognition: evidence for face expertise. J Exp Psychol Gen, 130(3), 534-543.

Tanaka, J. W., \& Farah, M. J. (1993). Parts and wholes in face recognition. Q J Exp Psychol A, 46(2), 225-245.

Valentine, T. (1988). Upside-down faces: a review of the effect of inversion upon face recognition. Br J Psychol, 79(Pt 4), 471-491.

Vuilleumier, P. (2005). Cognitive science: staring fear in the face. Nature, 433(7021), 22-23. doi: 10.1038/433022a

Yin, R.K. (1969). Looking at upside-down faces. Journal of Experimental Psychology, 81, 141-145. 
Zychaluk, K., \& Foster, D. H. (2009). Model-free estimation of the psychometric function. Atten Percept Psychophys, 71(6), 1414-1425. doi: 10.3758/APP.71.6.1414 


CHAPTER 3

\section{ATTRIBUTE-SELECTIVE ADAPTATION}

Category-specific Effect of Adaptation on Faces and Objects 


\section{ABSTRACT}

The influence of recent exposure on subsequent perception - known as the adaptation after-effect- has been used as a powerful tool to study the nervous system. In the present study, we introduce the Attribute Selective Adaptation (ASA) as a novel extension of the classical adaptation paradigm. ASA is based on the rapid presentations of numerous partially-overlapping stimuli in the adaptation phase, with different low-level features, but sharing the same highlevel attribute. We suggest that adaptation effects during ASA are more specific for high-level attributes than in conventional adaptation paradigms.

In a series of four psychophysical experiments in humans, we employed ASA in face- and object detection tasks with object category as the attribute of interest. Adaptation was measured as reduced efficiency in detecting an object in noise. Experiments 1-3 revealed a category-specific effect of adaptation for face adaptors, in line with previous research. In addition, we demonstrated a smaller but significant cross-category effect of adaptation. In the fourth experiment, we continued with a similar ASA in a shoe detection task. The results revealed similar category-specific effects of adaptation as in the face detection task, but no cross-category adaptation effect to non-face objects. Taken together, the experiments showed that face detection is affected by high-level adaptation to both face and non-face categories, whereas object detection is only affected by adaptation to the within-category objects.

Keywords: Attribute-Selective Adaptation; ASA; face detection; object categorization 


\section{1| Introduction}

The temporal history of prior exposures to a stimulus affects the neural representation of that stimulus and hence our perception of it (for review, see Shwartz et al., 2007). The impact of recent temporal history has been a powerful tool to investigate how the nervous system works. In vision, it has long been known that prolonged exposure to a stimulus can alter the perception of the subsequent stimulus, known as the adaptation aftereffect (Gibson \& Radner, 1937). The effect of adaptation can be either to shift the perception of a test stimulus away from an adapted stimulus (negative after effect) or to shift the perception of a test stimulus towards the adapting stimulus, on a certain stimulus dimension. Studying adaptation helps us to understand the coding principles underlying the perception of certain stimulus dimensions (e.g., in this case supporting the idea of channels (De Valois, Albrecht, \& Thorell, 1982)). Adaptation aftereffects have also been used in various other sensory modalities such as olfactory (Ekman, Berglund, Berglund, \& Lindvall, 1967) and auditory (Schweinberger et al., 2008).

In recent years, the adaptation paradigm has also been used to explore high-level processes such as face perception. One of the first studies on highlevel adaptation demonstrated that adapting to distorted faces can influence the perception of subsequent normal faces (Webster \& Maclin, 1999). The authors showed that after long exposure to a distorted faces, the viewers perceived the original (non-distorted) stimuli as distorted in a direction opposite to the adapting distortion.

Nevertheless, adaptation paradigms have seldom been used in basiclevel object categorization experiments. In a recent study, it was shown that the object categorization boundary between two natural objects was shifted by long exposure to one or the other of the two objects, while categorizing the ambiguous morphs between the objects (Daelli \& Treves, 2010). In the same study, the time interval between the onset of the test stimulus and the offset of the adaptation stimulus was also manipulated. The study showed that the perceptual shift can be either away from the adaptor for short intervals or towards the adaptor for long intervals. Importantly, to show that the observed effect was due to high-level category-dependent processes, the authors used the test stimulus in two size categories: (a) same size as the adapting stimuli; (b) a larger size compared to the adapting stimuli. In this way, they tried to rule out that the observed adaptation effect would be due to low-level processes. 


\section{Chapter 3}

Adaptation has not only been a popular tool in behavioral research, it also has been applied widely in $\mathrm{fMRI}$ research to determine the contribution of cortical regions to the processing of both lower-level and high-level stimulus attributes (Kalanit Grill-Spector \& Malach, 2001; Kourtzi \& Grill-Spector, 2005). Just as in behavioral studies, most fMRI studies measured adaptation in response to repeated exposure of the same stimulus. Neurophysiological studies have put into question how to interpret the results of adaptation in a given area in such adaptation paradigms, because of the difficulty to separate any adaptation effects within circuitry in the area of interest from adaptation of the input (Logothetis, 2008; Sawamura, Orban, \& Vogels, 2006).

In the present study, we introduce "Attribute-Selective Adaptation" (ASA) as an extension of the conventional adaptation paradigm as a tool to limit adaptation to targeted mid/high-level visual processes. The adaptation phase in ASA entails rapid presentation of numerous stimuli sharing the same attribute, which we call the attribute of interest. In the present study, the attribute of interest in the different reported experiments is the object category (face-ness and shoe-ness). To tap into the position-invariant representations of the attribute of interest, we presented the adapting stimuli at a rapid rate in random locations with partial overlap within the region of adaptation. It is important to mention that the stimuli did not disappear upon the presentation of the next stimulus but were just partially occluded. This prevents an illusion of motion. The position randomization would ensure that low-level features present in the stimuli would not lead to repeated stimulation of neurons in lowlevel areas characterized by small RFs (R Gattass, Gross, \& Sandell, 1981; Hubel \& Wiesel, 1962). The position randomization however does lead to repeated stimulation of neuronal mechanisms sensitive to mid-to high level aspects of objects in mid- to high level visual areas where receptive fields are large ( $R$. Gattass, Sousa, \& Gross, 1988; Gross, Bender, \& Rocha-Miranda, 1969). Following the adaptation phase there is a test phase, which is similar to that used in conventional adaptation experiments in which the performance is measured by means of ambiguous objects (Clifford \& Rhodes, 2005). However, unlike most conventional studies, the test stimulus is presented outside of the adaptation location. We believe that this paradigm is suitable to isolate highlevel processes for two reasons: (a) it taps into the position-invariant representations of the object during the adaptation period and in the test phase; (b) instead of one instance of the adaptor per trial, it overflows the visual system with hundreds of instances of adapting stimuli from the same class. In the present study, we report on 4 experiments designed to test the 
suitability of ASA for use in adaptation studies, and to demonstrate that the new paradigm shows the adaptation effects that have been reported also with more classical adaptation paradigms.

\section{The Goal of the Present Study}

In the current study, we examined the adaptation effect of ASA with face and one non-face object category as adaptor in face and object detection tasks. The participants were asked to perform the detection tasks with spatial frequency degraded stimuli compared with non-adaptation condition. We also compared the high-level adaptation effect of face and a non-face (shoe) category on detection performance in face and non-face detection tasks.

\section{2 $\quad$ General Methods and Materials}

\section{Participants}

In Experiment 1, we recruited 10 participants (7 females; age range: 18-32) from Tilburg University. We recruited 10 participants (8 female; age range: 18-24) for Experiment 2, 20 participants (15 female; age range: 18-26) for Experiment 3, and 20 participants (13 female; age range: 18-24) for Experiment 4 from Maastricht University. All participants were right-handed, with normal or corrected-to-normal vision and naive as to the purpose of the experiments. The study was conducted in accordance with local ethics committee requirements. The participants gave informed consent to participate and were rewarded with either one course credit or a 7.5 Euro voucher.

\section{Stimulus Preparation}

Adaptors. Original face images were color images of 33 neutral faces (16 females). Three of the face images were used to generate the noisy test images and the rest were modified to be presented as adapting stimuli. All of the images were selected from a sub-set of the Karolinska Directed Emotional Faces (KDEF) database (Lundqvist, Flykt, \& Öhman, 1998). For the shoe stimuli used in the adaptation phase we selected 31 shoe images from our own shoe database (de Gelder, Bachoud-Levi, \& Degos, 1998). The stimuli were preprocessed as follows: first, the original images were cropped and resized to fit into a rectangular format- using Adobe Photoshop CS6 (Adobe; http://adobe.com). Then, average pixel values for each image were shifted to 


\section{Chapter 3}

128 in Matlab 2009a (Mathworks; http://www.mathworks.com). The dimensions of the adapting stimuli on the screen were $3.8^{\circ} \times 5.1^{\circ}$.

Test stimuli. Noisy stimuli were generated by randomizing the phases of Fourier transform of the preprocessed face stimuli while keeping the Fourier power constant (Figure $1 \mathrm{E}$ ). The phase shuffling is quantified by a percentage signal, in which zero means all phase information has been randomized, preserving the overall distribution, and in which 100 means all phase information is intact (Goffaux et al., 2011; Mack, Gauthier, Sadr, \& Palmeri, 2008; Sadr \& Sinha, 2001). The main advantage of this technique is that it maintains the significant components for low-level vision (such as average luminance and Fourier power distribution) constant. The original images were selected from the same datasets from which we chose the adaptors with different identities from the ones we selected before. The test face stimuli were generated at signal percentages of 50,60,70, 80 and 90. The test shoe stimuli were generated at signal percentages of $20,30,40,50,60,70,80$ and 90 . The dimensions of the test stimuli were the same as the adapting stimuli $\left(3.8^{\circ} \times 5.1^{\circ}\right)$.

Mask stimuli. The mask stimuli were checkerboard patterns with the same size of the test stimuli. Each square of the pattern subtended $0.31^{\circ}$. The sole purpose of using checkerboard patterns was to suppress the image aftereffect of the test stimuli. The pilot study prior to the experiment showed that such an effect can be used as one of the main cues for the participants and might confound the findings.

\section{Experimental Procedure}

Each experimental session consisted of multiple adaptation and non-adaptation blocks (Figure $1 \mathrm{~A}$ ). The non-adaptation trials consisted of only a test phase. The trials in the adaptation blocks consisted of one adaptation phase followed by a test phase. In all experiments, there were random inter-trial-interval (ITI) between 500 and $650 \mathrm{~ms}$.

Test phase. The test phase consisted of $500 \mathrm{~ms}$ of fixation followed by a test stimulus (Figure $1 \mathrm{C}$ ). The test stimulus was presented for $100 \mathrm{~ms}$ on the horizontal meridian on the right or left side of the fixation cross (counterbalanced). The randomization of the presentation site in the test phase was originally designed to minimize the chance of unwanted predictive saccades to the test location. We were also interested in examining the possibility of any 
left visual field advantage over the right one in the detection tasks (Moscovitch \& Radzins, 1987; Rhodes, 1985). The test stimulus was immediately masked by a checkerboard pattern for $250 \mathrm{~ms}$. The distance between the fixation cross and the center of the test stimulus was set to $7.5^{\circ}$ (Figure 1D). Next, the response screen appeared and the participant's task-relevant response by pressing the appropriate mouse key. In Experiments 1-3 the left button was associated with face and the right press was corresponding with the no-face response. In Experiments 4 the left button was associated with shoe and the right press was corresponding with the no-shoe response.

Adaptation phase. In the adaptation phase, each adapting stimulus was presented for $33 \mathrm{~ms}$ followed by another adapting stimulus. The adaptation phase consisted of approximately 5 pseudorandom orders of 30 different identities (faces in experiments 1-3; shoes in Experiment 4), presented in successive cycles for a total duration of 5 seconds (Figure 1B).

The fixation cross was always visible on top of the adapting stimuli. The adapting stimuli were presented in random locations within a hypothetical square centering on the fixation cross. Note that the adapting stimuli were not disappearing after presentation but were partially occluded by each new adapting stimulus. This was implemented after pilot experiments in order to prevent the perception of movement, due to the high rate of stimulus presentation. The centers of the adapting stimuli were randomly chosen within a maximum distance of $2.2^{\circ}$ from the fixation; thus the adapting stimuli were always overlapping spatially.

Furthermore, in Experiment 4 where the test stimuli covered a larger range of signal levels, we performed psychometric fitting. For this, the behavioral performance was plotted separately for each of the experimental conditions in each participant. Data points were fitted by a logistic cumulative distribution function:

$$
\mathrm{f}(\mathrm{x} ; \alpha, \beta)=\frac{1}{1+\exp (-(\mathrm{x}-\alpha) / \beta)},
$$

where $x$ is the stimulus signal proportion (1- noise ratio added to the original stimulus) and $f(x)$ is the probability of the shoe response. Every psychometric function was achieved, fitting logistic regression with 2000 bootstrap iterations, without prior assumptions on the parameters of the underlying function using the psignifit toolbox version 2.5.6 (Graham, 2001) for Matlab (www.mathworks.com) which implements the maximum-likelihood method described by Wichmann and Hill (Wichmann \& Hill, 2001). 


\section{Chapter 3}

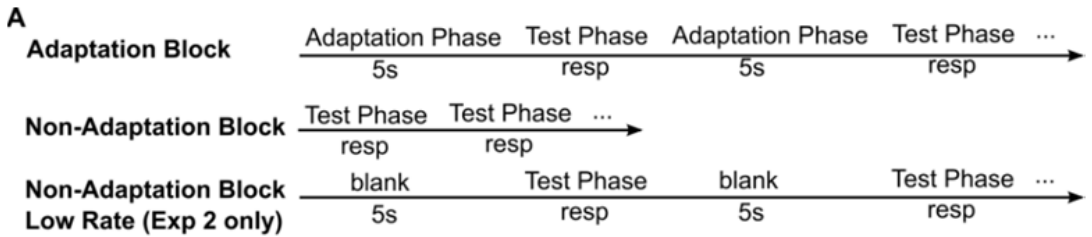

B

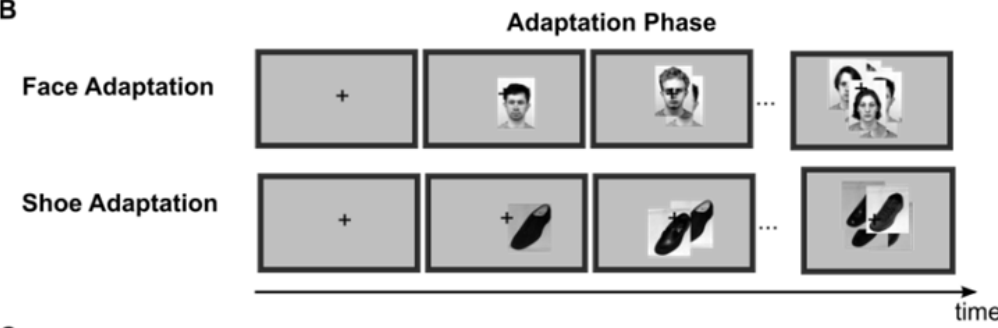

C

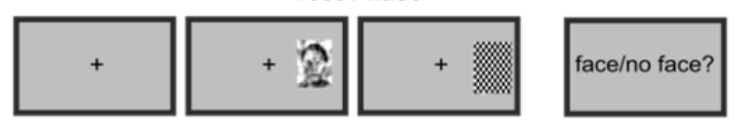

Face Test

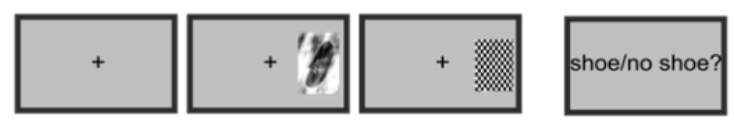

Shoe Test
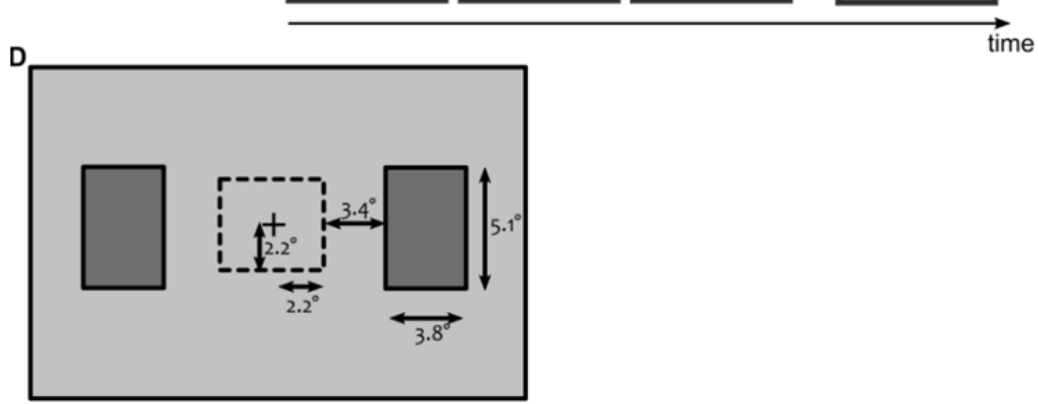

E
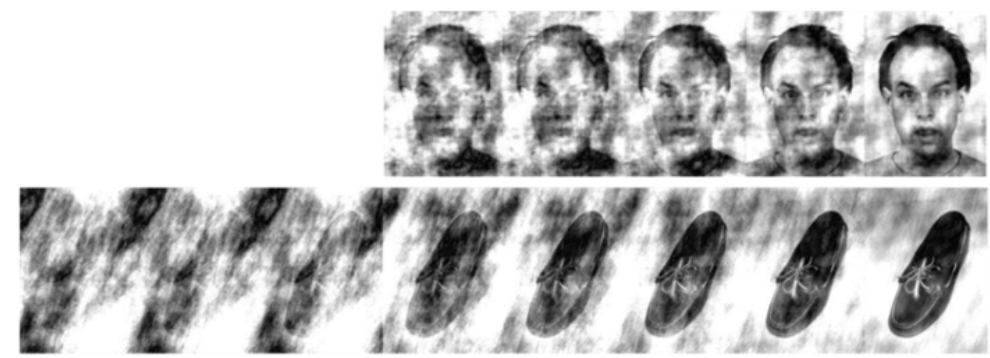

$\%$ Sig 20

30

40

50

60

70

80

90

Figure 1 Overview of experimental design and stimuli. (A) Schematic view of experimental procedure in adaptation and non-adaptation blocks. In adaptation blocks, ASA consists of the adaptation phase, followed by the test phase; the non-adaptation blocks consisted of test phase only. In Experiment 2, we also included a condition of low-rate non-adaptation condition in which the test phase appeared 
at the same rate of the adaptation condition; thus, every trial consisted of 5 seconds of pre-stimulus blank screen. (B) Schematic view of the adaptation phase: The adaptation phase consists of numerous stimuli from the same attribute (here, object category), each presented for $33 \mathrm{~ms}$. The total duration of the adaptation phase is approximately 5s. (C) Schematic view of the test phase: In the test phase, the test stimulus is presented for $100 \mathrm{~ms}$, immediately followed by a checkerboard pattern for $250 \mathrm{~ms}$ before the response screen appears. (D) Overview of the stimulus dimensions and distances (visual degrees) in all experiments: the dashed square is a hypothetical area in which the centers of the adapting stimuli are randomly located; the two dark rectangles-symmetrical to the fixation cross- represent the test stimulus. In each trial one of the two sites is chosen randomly for the presentation of both the test and the mask stimuli. (E)Test stimuli at different levels of signal (see 'stimulus preparation').

\section{3| Experiment 1: Adaptation to Faces vs. No Adaptation}

We examined whether there was any difference in face detection performance when comparing face detection on adaptation trials with face detection in nonadaptation trials. To implement adaptation in the adaptation trials, we first rapidly presented hundreds of different face stimuli, in random locations around a central fixation point. In the subsequent test phase, we measured the detection performance at different levels of signal for the test stimuli using a forced-choice paradigm.

\section{Methods and Materials}

Stimuli. We used a total of 30 (15 female) preprocessed (see General Methods and Materials) face images for the adaptation phase and 5 signal levels of another face identity for the test phase. We also used two shoe stimuli at $50 \%$ noise as catch stimuli ( $28 \%$ of all trials).

\section{Results and Discussion}

We first examined the possible response bias for the face response by averaging the proportion of responses to the catch trials (scrambled shoe stimuli) across the participants. The results showed above $96 \%$ accuracy in correctly rejecting the catch trials for all participants. Then, we averaged the proportion of response to all 5 signal levels of the face image in each experimental block and for every participant.

As presented in Figure $2 \mathrm{~A}$, the initial results showed strictly increasing face response as a function of signal level. Furthermore, the graphs revealed a clear negative adaptation effect by the lowered face responses compared to non-adaptation blocks, suggesting an increased perceptual threshold for face 


\section{Chapter 3}

detection. It also showed a declining trend for the non-adaptation condition along the experimental session.
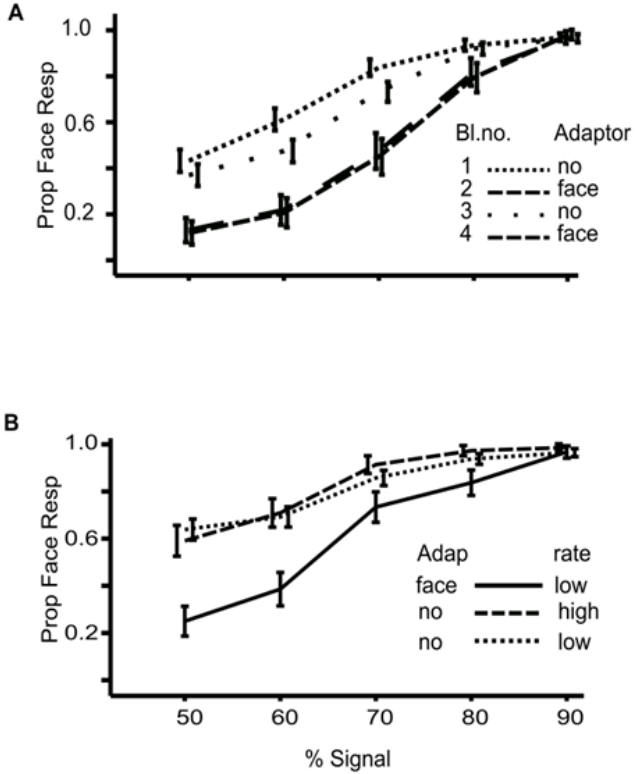

Figure 2 Proportion of face responses relative to the percentage signal. (A) Average face response in Experiment 1 for each experimental block; (B) Average face response in Experiment 2 for the faceadaptor condition and two non-adaptation conditions. Error bars represent $95 \%$ confidence interval.

Next, we examined the difference in the responses for different conditions. For this, we entered the five signal levels, the two test presentation locations (left and right), the adaptation conditions (face adaptors versus no adaptor), and the block order as within-subject variables in a repeated measure analysis of variance (ANOVA). The results showed a significant effect for signal level $(F(1.61,14.51)=78.87, p<0.05)$ (Figure $2 A)$. There was also a significant main effect of adaptor $(F(1,9)=33.49, p<0.05)$. Furthermore, the interaction between signal level and adaptor was significant $(F(2.54,22.92)=12.10)$. There was no significant difference between the first and the second blocks of the two tasks and between the two presentation locations (left and right) and their interactions ( $p>0.05)$. The estimated effect of adaptor was calculated as the mean difference between the adaptor conditions $\left(I_{\text {no-adap }}-J_{\text {sho-adap }}=0.212 \pm\right.$ 0.037).

Experiment 1 demonstrated that the adaptation phase can disrupt the perception of the subsequent spatial-frequency-noised faces presented 
peripherally. A plausible explanation for this finding may be that there was high level visual adaptation across the category-specific attributes of the face stimulus. However, there are some limitations to this interpretation. First, it is possible that the observed effect was restricted to the specific face images used as a test stimulus. Second, it is possible that the observed effect was due to the difference in the response rhythms in adaptation and no-adaptor trials. As the no-adaptor trials only included a test phase, responses followed each other much faster in the non-adaptation condition, which might have contributed to better performance compared to adaptation condition. These concerns were tested in the next experiment.

\section{4| Experiment 2: Effect of Target Rate on the Adaptation Effect}

In the second experiment we investigated whether the observed effect in Experiment 1 was due to the difference in the rhythm at which the test phases were presented in adaptation and non-adaptation trials. It is known that fastpaced trials might lead to a better performance in certain experimental conditions due to the temporal attention span (De Jong, Berendsen, \& Cools, 1999; Kane \& Engle, 2003). To that aim, we modified the non-adaptation blocks by presenting a blank screen between every two trials for the same duration of the adaptation phase in the adaptation blocks; hence, the test stimuli appeared with the same rate in both non-adaptation and adaptation tasks. We also examined two other face identities at similar levels of noise to experiment 1 to confirm that the observed effects in Experiment 1 were not due to certain physical properties of the original face image identity from which the test stimuli were generated (see General methods and materials).

\section{Methods and Materials}

Stimuli. The adaptation stimuli were the same as those used in Experiment 1. The test stimuli were two identities ( 1 female) at 5 different levels of noise. The two test identities were different from the one used in Experiment 1.

Procedure. The experimental procedure was the same as that in Experiment 1 with the only difference that a 5 second blank screen was inserted in the non-adaptation condition in order to make the two tasks more 


\section{Chapter 3}

comparable in the sense of rhythmicity. Each of the adaptation and nonadaptation blocks consisted of 8 repetition of each face signal level presented on each of the two presentation sites.

\section{Results and Discussion}

First, we averaged the proportion of responses for each presentation location and each signal level in the adaptation task and in each of the two no adaptation (low rate and high rate) tasks. Then, we compared all conditions using a repeated measure ANOVA.

As shown in Figure $2 \mathrm{~B}$, there was a significant effect of signal level ( $\mathrm{F}$ $(1.24,11.15)=44.18, p<0.05)$. The results showed no significant difference between the long and short durations of the fixation $(F(1,9)=0.212, p>0.05)$. Also, the interaction of signal level and task turned out to be not significant ( $F$ $(4,20.13)=3.27, p>0.05)$. Furthermore, there was no significant difference between the two sides (left and right) of presentation $(F(1,9)=3.68, p>0.05)$. Afterwards, we ran pairwise comparisons of the signal levels with Bonferroni correction. The tests showed that all signal levels were significantly different from each other. We then calculated the estimated marginal means of the difference -as the size of adaptation effect- between the non-adaptation (shortfixation) and face-adaptation tasks ( $I_{\text {no-adap }}-J_{\text {shoe-adap }}=0.22 \pm 0.033$ ). By doing this, we could quantitatively compare the effect of adaptation in Experiments 1 and 2 and see whether the difference in the face identities lead to any difference in the effect of adaptation. The results showed no significant difference $(p>0.05)$ between the two experiments.

The obtained results showed that the observed effect in Experiment 1 was due neither to a faster rate of the presentation of test stimuli in the nonadaptation than in the adaptation conditions, nor to the specific identity of the test stimuli. Therefore, in the rest of the experiments, we returned to the trial structure of Experiment 1 (with shorter adaptation trials (non-adaptation highrate) and the use of a single face testing stimulus at different levels of noise without catch trials).

\section{Experiment 3: Face detection after Adaptation to Faces and Adaptation to Shoes}

In Experiment 3, we examined whether the effect observed in Experiments 1 and 2 is specific to the category of the adaptor. As in the previous experiments, the test phase required participants to detect faces in stimuli deteriorated by 
different levels of noise, but her the preceding adaptation phase could include either exposure to faces or to shoes. This condition is comparable to the baseline experiment in Daelli et al., where they only compared the effect of the conventional adaptation condition to the same-category test stimulus with the adaptation to another category. We here used both non-adaptation condition (as in experiments 1 and 2) plus a cross-category adaptation-similar to their paradigm- to see if there is also any significant perceptual shift if the participants are adapted to another object category compared to the nonadaptation condition. In other words, we aimed to verify if the cross-category adaptation -instead of non-adaptation condition- can be taken as a baseline.

\section{Methods and Materials}

Stimuli. The face adaption and the test stimuli were the same as those used in Experiment 1. For Shoe-adaptation condition we used 30 preprocessed shoe images (see General Materials and Methods).

Procedure. The experimental procedure consisted of 5 experimental blocks. Blocks number 1,3 and 5 were non-adaptation conditions. Block numbers 2 and 4 were face adaptation and shoe adaptation conditions, counter-balanced across the participants. Each experimental block consisted of 8 repetitions of each face signal level and the presented on each of the two presentation sites (left and right). Each non-adaptation block consisted of 8 repetition of each stimulus and the two catch stimuli presented on each of the two presentation sites. Since the adaptation blocks took more than two times longer than the no adaptation blocks ( $\sim 5 \mathrm{~min})$, both adaptation blocks were divided into two sub-blocks with a two minute break in between. This was done to rule out general exhaustion as a reason for the decline of detection performance in the adaptation conditions.

\section{Results and Discussion}

First, we looked at the three blocks devoted to the non-adaptation condition to see if there was any effect of order. Figure 3 A shows highly overlapping results for the three no adaptation blocks. This was confirmed by a repeated measure ANOVA in which block order number was used as a factor. The results showed no significant effect of order $(F(1.12,13.50)=0.19 ; p>0.05)$ (Figure $3 A)$. Thus, we averaged the three runs as the non-adaptation condition for further analysis (Figure 3B). Figure 3B shows detection performance in the test phase in trials 


\section{Chapter 3}

that included adaptation to faces, to shoes, or no adaptation. The data confirm a large reduction of face detection performance in the face adaptation condition compared to the no adaptation condition. Interestingly, adaptation to shoes also seemed to have resulted in a small effect on face detection. The side of presentation of the test stimuli, left or right, see Figure $3 \mathrm{C}$ ) had no effect. To test these observations, we ran a repeated measure ANOVA including signal level, side of image presentation, and adaptor condition (non-adaptation, faceadaptor and shoe-adaptor) as factors. We found a significant effect of signal level $(F(2.13,38.43)=94.97, p<0.05)$, of and adaptor type $(F(2,36)=53.19, p<$ $0.05)$. However, there was no difference between the two testing locations ( $F$ $(1,18)=0.02, p>0.05)$. There was also a significant interaction between signal level and adaptor $(F(3.33,60.01)=7.79, p<0.05)$. All other interactions were not significant $(p>0.05)$.

We then performed a pairwise comparison with Bonferroni correction for the signal levels and adaptor conditions. The estimated marginal means of differences, regarded as size of the effects, revealed also a small but significant difference between the non-adaptation and shoe-adaptation conditions ( $I_{\text {no-adap }}$ $\left.-J_{\text {shoe-adap }}=0.081 \pm 0.026, p<0.05\right)$. The within-category adaptation, however showed a larger effect compared to the no adaptation condition ( $I_{\text {no-adap }}-J_{\text {face- }}$ adap $=0.261 \pm 0.030, p<0.05)$, and to the shoe-adaptation one $\left(I_{\text {shoe-adap }}-J_{\text {face- }}\right.$ adap $=0.180 \pm 0.030, p<0.05)$. We further post-hoc compared every response pair for different adaptor conditions for every signal level due to the significant interaction effect between adaptor and signal level, suggesting different behavior of the response across signal levels. The analysis showed significant difference between all the three adaptor conditions after correction for multiple comparisons for the two lowest signal levels of $50 \%\left(I_{\text {no-adap }}-J_{\text {face-adap }}=\right.$ $\left.0.417 \pm 0.043, p<0.05 ; I_{\text {shoe-adap }}-J_{\text {face-adap }}=0.240 \pm 0.053, p<0.05\right)$ and $60 \%\left(I_{\text {no- }}\right.$ adap $-J_{\text {face-adap }}=0.416 \pm 0.044, p<0.05 ; I_{\text {shoe-adap }}-J_{\text {face-adap }}=0.247 \pm 0.050, p<$ $0.05)$. However, for the middle signal level (70\%) the difference between nonadaptation and face adaptation stayed significant but the difference between non-adaptation and shoe-adaptation conditions diminished $\left(1_{\text {shoe-adap }}-J_{\text {face-adap }}=\right.$ $\left.0.288 \pm 0.055, p<0.05 ; ; I_{\text {shoe-adap }}-J_{\text {face-adap }}=0.047 \pm 0.044, p>0.05\right)$. None of the pairwise comparisons were significant for the two highest signal levels $(80 \%$ and $90 \%$ ). The results from Experiment 3 suggest that the adaptation effect induced by ASA for the face test stimuli has two components: one is the overall effect of adaptation compared to non-adaptation condition regardless of the object category of the adaptor; the other effect is the same as the category- 
specific effect. This is an important finding since in cross-category adaptation one category is often used as a baseline for the other.

\section{6| Experiment 4: Effect of Adaptation to Shoes and Faces on the Detection of Shoes}

The purpose of this experiment was to investigate whether the observed adaptation effect in Experiment 3 for face test stimuli could be extended to other visual object categories. To that aim, we used adaptation trials with shoes and faces as adaptors, using the same ASA procedure, followed by a test phase using degraded shoe stimuli. Trials without adaptation phase were used as control. Furthermore, since in Experiment 3 we observed that adaptation spread to some extent from faces to shoes in a detection task, we wondered if the same effect exists when adapting to faces and detecting shoes.

\section{Methods and Materials}

Stimuli. We used a shoe identity presented at different signal levels as test stimulus. We also extended the number of signal levels to cover the whole psychometric behavior spectrum. By this, we could further observe the psychometric changes as a result of adaptation to each object category. The adaption stimuli were the same as the ones used in Experiment 3. The test stimuli were 8 different levels of noise of one shoe stimulus, which was not included in the shoe-adaptation condition.

Procedure. The experimental procedure was similar to the one in Experiment 3. The order of the adaptation blocks was different in the present experiment compared to Experiment 3. As a result, the second and fourth blocks were shoe- and face adaptation tasks respectively. This was done to keep the within- and cross-category order the same.

Data analysis. First, the data were analyzed using repeated measure ANOVA, similar to experiments 1-4. Then the behavioral responses were plotted separately for each of the experimental conditions and were fitted by a logistic cumulative distribution function. Then, the slope and the thresholds at 50\% performance were calculated for each adaptor type in every participant. This was possible regarding the wider range of signal levels which made it possible to obtain a full range of response proportions between 0 and 1 . Finally, the 


\section{Chapter 3}

thresholds and slopes were compared using repeated measure ANOVA. The latter analysis could be more sensitive to the differences that were not detected using ANOVA with signal level as one of the factors.

A
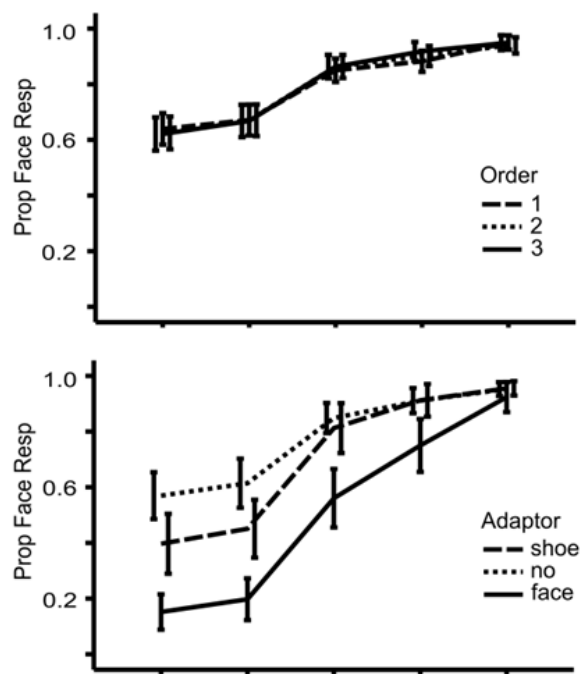

C

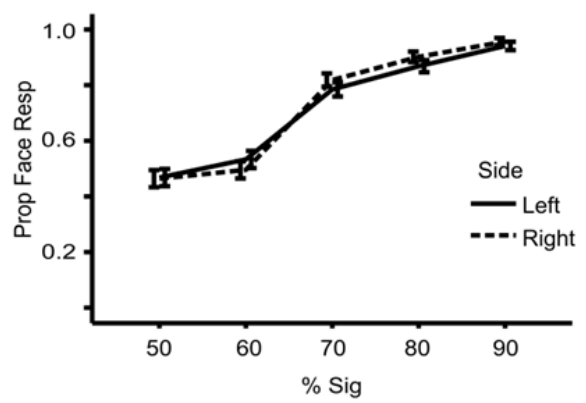

Figure 3 Proportion face response in Experiment 3. (A) Average responses in face detection task for each of the 3 blocks of the non-adaptation condition; (B) Average face detection performance for each of the three (non-adaptation, face-adaptation and shoe adaptation) conditions; (C) Average face detection performance for each presentation site across all conditions. Error bars represent $\mathbf{9 5 \%}$ confidence interval.

\section{Results and Discussion}

We ran three blocks of the non-adaptation condition with one of the two adaptation blocks in between each two consecutive non-adaptation blocks. We 
examined the effect of order to determine whether participants' overall performance changed during the experiment. Figure 4A suggests a small difference between the first and the next two runs. To test this difference, we fed the three runs into a repeated measure ANOVA with order number as factor. The results showed a significant effect of order across the nonadaptation conditions $(F(1.26,11.33)=9.80, p<0.01)$.

A

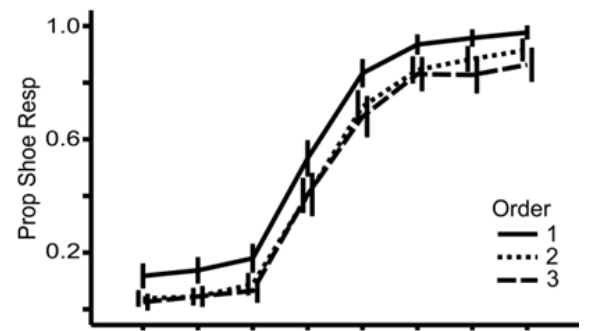

B

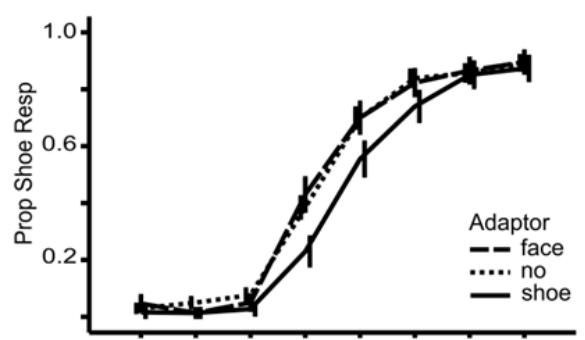

C

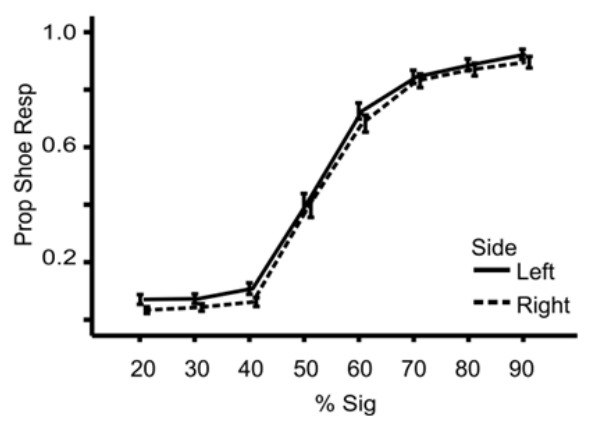

Figure 4 Average responses in the shoe detection task: (A) overall change of the shoe detection task during the experimental session; (B) shoe detection performance as a function of signal level for different adaptation conditions; (C) average shoe detection performance in each of the presentation sites. Error bars represent $\mathbf{9 5 \%}$ confidence interval.

Moreover, the pairwise comparisons with Bonferroni correction showed a significant difference between the first and each of the two other runs $\left(I_{\text {no-adap } 1}-J_{\text {no-adap } 2}=0.10 \pm 0.04, p<0.05 ; I_{\text {no-adap } 1}-J_{\text {no-adap } 3}=0.12 \pm 0.04, p<\right.$ 


\section{Chapter 3}

0.05). However, there was no significant shift between the two latter runs $\left(I_{\text {no- }}\right.$ adap $\left.2-J_{\text {no-adap } 3}=0.02 \pm 0.02, p>0.05\right)$. Since the adaptation blocks were number 2 and 4 , we took the non-adaptation blocks numbers 3 and 5 as stable controls and excluded block 1 and used the average of the other two runs as nonadaptation condition.

Figure $4 \mathrm{~B}$ shows a clear shift of the psychometric curve towards worse performance in the test phase of shoe detection trials when the preceding adaptation was to shoes. However, shoe detection was unaffected by preceding adaptation to faces. Figure $4 \mathrm{C}$ also shows that there was no difference between the two locations used for testing. To test the significance of these observations, we compared the effect of the three factors (signal levels $\mathrm{x}$ presentation sites $\mathrm{x}$ adaptor conditions) using repeated measure ANOVA as shown in Figure 4B. The effect of signal level was significant, ( $F(2.28$, $38.79)=298.53, p<0.05)$. Furthermore there was a significant main effect of adaptor condition $(F(2,34)=15.34, p<0.05)$. In addition, the interaction of signal level and adaptor condition was significant $(F(6.11,103.90)=5.23, p<$ $0.05)$. There was no significant effect of presentation location. The rest of the interaction effects were not significant $(p>0.05)$. Further analysis of estimated marginal means differences with Bonferroni correction showed that all three adaptor conditions were significantly different $\left(I_{\text {no-adap }}-J_{\text {face-adap }}=0.008 \pm 0.016\right.$, $\left.p>0.05 ; I_{\text {no-adap }}-J_{\text {sho-adap }}=0.068 \pm 0.019, p<0.05\right)$. The interaction between the adaptor and signal level was significant as well $(F(6.1,104.01)=4.90, p<0.05)$. The lack of a significant effect $(p>0.05)$ of either presentation site or its interactions with other factors was confirmed (Figure $4 \mathrm{C}$ ). We also calculated the estimated marginal mean of differences between each pair of conditions only for the signal levels overlapping with Experiment 3 (50, 60, 70, 80, and 90). The results still showed a significant same-category adaptation effect and no significant difference between the non-adaptation- and cross-category adaptation conditions $\left(I_{\text {no-adap }}-J_{\text {face-adap }}=0.261 \pm 0.030, p>0.05 ; I_{\text {no-adap }}-J_{\text {sho- }}\right.$ adap $=0.081 \pm 0.026, \mathrm{p}<0.05)$.

\section{7| General Discussion}

In present study, we introduce Attribute Selective-Adaptation (ASA). This is a novel adaptation paradigm, which we suggest is more suitable for studying specific contributions of high-level visual areas to encoding of high-level object features and categorization. Our approach is new in the sense that the participants were adapted to a specific attribute instead of a specific stimulus, 
and in the sense that adaptation took place well away from the location where adaptation took place. Our primary goal was to show that this paradigm yields adaptation effects that are comparable to what can be expected from existing literature using more commonly used adaptation procedures (i.e., without spatial randomization and without ensuring non-overlapping positions for adaptation and testing)(Ghuman, McDaniel, \& Martin, 2010; Kovacs et al., 2006; Leopold, O'Toole, Vetter, \& Blanz, 2001). Our first two experiments demonstrated the robustness of adaptation effects in different experimental designs. In experiments 3 and 4, we further investigated whether the samecategory and cross-category adaptation effect is the same as the nonadaptation for two categories (faces and shoes). The results interestingly showed that face detection was affected by adaptation to both faces and shoes but shoe detection was only influenced by adaptation to shoes and not faces.

\section{Object Categorization and the Role of Spatial Frequency}

Previous research investigating high-level adaptation has been mostly centered on the domain of face processing. Such studies focused on identity (Leopold et al., 2001), age, gender, ethnicity, expression (Webster, Kaping, Mizokami, \& Duhamel, 2004), etc. However the effect of adaptation has not been behaviorally characterized in the more basic process of face detection. Furthermore, to our knowledge only one recent study has investigated the adaptation of complex non-face objects (Daelli \& Treves, 2010). They examined the effect of adaptation on object categorization boundaries across the morphing continuum of pairs of natural objects. Their results showed that the object categorization boundary was shifted by long exposure (3 seconds) to each of the two endpoint objects. They also demonstrated that there is a negative adaptation effect for the short intervals between the adaptor and the test stimulus (similar to the present study). Furthermore, they examined the level of the adaptation effect by using different object sizes between the adaptors and the test stimuli. Their approach is especially interesting where they explain their findings in the framework of attractor network models (Daelli \& Treves, 2010). In the present study, we extended such findings to the position-invariant effect of adaptation on more basic processing levels of faceand shoe detection tasks (instead of categorization). The findings of the current study can be generalized towards other object categories and face subcategories (e.g. expressions, gender, etc.). 


\section{Chapter 3}

Spatial frequency (SF) channels play a fundamental role in early visual processing (Georgeson \& Ruddock, 1980). However, the input from SF channels to higher processing levels also influences high-level processes (Torralba \& Oliva, 2003). For example, a number of aspects of face processing have been shown to depend on spatial frequency content (Goffaux, 2009; Goffaux, Gauthier, \& Rossion, 2003; Goffaux, Hault, Michel, Vuong, \& Rossion, 2005). In the present study, we ruled out effects of spatial frequency by keeping spatial frequency content constant across stimuli. Note that in Daelli et al. (2010), ruling out the spatial frequency involvement was more complicated due to the nature of the categorization tasks. The reason is that in such categorization tasks, two natural objects with completely different spatial frequency distributions are morphed to each other on a certain morph path. Morph path is the parametric weighting of the corresponding features in the two objects in order to generate the intermediate morphs. As a consequence, different morphing procedures result in different sets of ambiguous objects with different proportions of various spatial frequency channels. Probably one way to rule out this confounding factor is to run a similar experiment with initial images (before morphing) being preprocessed by the spatial frequency normalization method recently used by Xu and Biederman (2014).

\section{Asymmetry of the Adaptation Effect for Faces and Non-face Objects}

The dissimilarity between the effect of adaptation for faces and shoes on each other's detection looks paradoxical at first glance. A schematic interpretation of the results is shown in Figure 5A. The results suggest that the read-out mechanism for face detection is influenced by both object processing and face processing. Conversely, the read-out of non-face object detection is getting affected only by non-face object processing. Taking into account the neural correlates of face and object detection, this asymmetry can be explained to some extent.

First, we present the evidence showing that face detection can be influenced by non-face object processing (and hence by adaptation of the involved neural substrates). This corresponds to the arrow from shoe processing to face detection in Figure 5. Three types of evidence are relevant:

(a) Existence of a separate face detection module. The evidence for the existence of a separate processing unit for face detection comes from behavioral studies in prosopagnosic patients. It has been shown that such 
patients perform as good as normal controls in face detection tasks although they fail in face recognition and identification tasks. This suggests an independent face processing module at the level of detection.

(b) Fusiform face area (FFA) and face detection. On the one hand Kovács et al. have shown that the most robust position-invariant face representation is located in the fusiform face area (FFA) using an fMRI adaptation (fMRI-a) technique (Kovács, Cziraki, Vidnyánszky, Schweinberger, \& Greenlee, 2008). On the other hand Grill-Spector et al.(2004) showed that among all face selective voxels in the ventral stream, only FFA voxel activity correlates behaviorally with the face detection performance (K. Grill-Spector, Knouf, \& Kanwisher, 2004).

(c) FFA activation for non-face objects. Grill-Spector et al. (2004) demonstrated that half of the voxels in FFA get activated significantly by nonface objects (although to a lesser extent, which is still in line with our findings of less adaptation effect with shoes compared to faces).

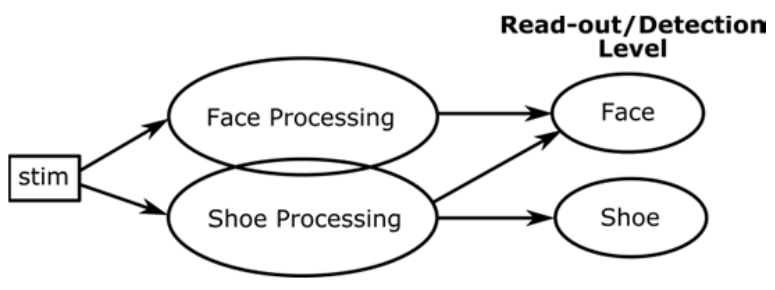

Figure 5 Schematic diagram of the results from experiments 3 and 4: Experiment 3 showed that face detection can be affected by both face processing and object (shoe) processing. Experiment 4 demonstrated that shoe detection is influenced by shoe processing but not face processing.

Second, we refer to the findings showing that face processing/adaptation does not influence subsequent object (here: shoe) detection (Experiment 4). This refers to the absence of an arrow between face processing and shoe detection in Figure 5. This idea is partially supported by the findings from Grill-Spector et al. (2004), who demonstrated that (non-face) object related activity in FFA is not correlated with (non-face) object detection performance. They also showed that there are voxels outside of FFA which get activated both by faces and object categories. Our findings predict that the object detection performance correlates only with the BOLD activity of the object-specific voxels and not the ones getting activated by both faces and objects. To our knowledge there is no experiment directly testing this hypothesis.

An important extension of the present study is to investigate how this asymmetric relationship can be extended to other similar natural objects while 


\section{Chapter 3}

being compared to faces. Our prediction is that a similar pattern will arise when comparing faces with other ordinary objects such as houses, trees, etc. However, we expect that in detection of expertise-related objects in the expert viewers (such as birds for birdwatchers) the pattern changes to a symmetrical effect of adaptation on detection of both faces and objects (Gauthier, Skudlarski, Gore, \& Anderson, 2000).

In general, our data can also contribute to the well-known bias of the nervous system to detect faces in the non-face objects, as soon as there is any hint of face-like features in those objects, or when stimuli are ambiguous (Hadjikhani, Kveraga, Naik, \& Ahlfors, 2009; Xu \& Biederman, 2014).

\section{Future applications of ASA}

The ASA paradigm introduced in the current research is technically important and can be used in the future studies on high-level visual attributes. This paradigm can also be useful in the neuroimaging studies focused on such attributes.

Position invariant effect of adaptation. One of the key features of ASA that makes it suitable for $\mathrm{mid} /$ high level visual processes is taking advantage of position invariant representations of the visual objects. Electrophysiological data shows that the neural RFs get larger as going up in the hierarchy (Afraz \& Cavanagh, 2008; Kovacs, Zimmer, Harza, Antal, \& Vidnyanszky, 2005; Lueschow, Miller, \& Desimone, 1994). This has inspired object- and face recognition models to include position-invariance as a key feature of the high-level processing ( $\mathrm{K}$. Grill-Spector, 2003; Riesenhuber \& Poggio, 1999).

In terms of ASA, two aspects could reduce the contribution of low level features to adaptation in high level areas: (1) the randomization of position in the adaptation phase; (2) the difference in position between adaptation stimuli and test stimuli. The latter has been previously used in some conventional highlevel adaptation experiments as a control for the same-location test in order to verify the portion of the high-level effects compared to the low-level ones (Daelli et al., 2010; Kovacs et al., 2005; Kovács et al., 2008; Zimmer \& Kovacs, 2011). Further investigations are required to compare the amount of adaptation in classical paradigms and ASA by parametrically reducing further the overlap in the adaptation phase and increasing distance between adapting- and testing stimuli. These two parameters will determine to what extent one excludes the contribution of the low level areas. Future research can also envisage extending 
the application of ASA to other high-level attributes. Moreover, additional stimulus aspects that could be randomized to increase specificity of adaptation include stimulus size and viewpoint of the adapting and the test stimuli.

fMRI-adaptation. One of the main future directions of the current study is to perform ASA together with advanced imaging techniques. The original functional magnetic resonance-adaptation ( $f M R I-a)$ paradigms rely on the differential BOLD activity of different stimulus sets using functional magnetic resonance $(\mathrm{fMRI})$. Few studies have already taken advantage of serial presentation of adapting stimuli and a series of test stimuli in a single experimental trial (Avidan, Hasson, Hendler, Zohary, \& Malach, 2002; Kourtzi \& Grill-Spector, 2005). In such fMRI runs (or events) the actual behavioral change caused by such adaptations could easily be measured. The ASA paradigm permits the behavioral measurement of adaptation to specific high-level features and we suggest it will permit a more specific interpretation of adaptation effects measured with fMRI.

\section{Acknowledgements}

This study was partly supported by Human Frontiers Science Program RGP54/2004, NWO Nederlandse Organisatie voor Wetenschappelijk Onderzoek 400.04081, and EU FP6-NEST-COBOL043403 and FP7 TANGO to Beatrice de Gelder, and NWO VICl grant to Peter De Weerd. We also thank Rebecca Watson for her suggestions on the manuscript. 


\section{References}

Afraz, S. R., \& Cavanagh, P. (2008). Retinotopy of the face aftereffect. Vision Res, 48(1), 42-54. doi: 10.1016/j.visres.2007.10.028

Avidan, Galia, Hasson, Uri, Hendler, Talma, Zohary, Ehud, \& Malach, Rafael. (2002). Analysis of the neuronal selectivity underlying low $\mathrm{fMRI}$ signals. Current biology, 12(12), 964-972.

Clifford, Colin WG, \& Rhodes, Gillian. (2005). Fitting the mind to the world: adaptation and after-effects in high-level vision.

Daelli, V., \& Treves, A. (2010). Neural attractor dynamics in object recognition. Experimental brain research, 203(2), 241-248. doi: 10.1007/s00221-0102243-1

Daelli, V., van Rijsbergen, N. J., \& Treves, A. (2010). How recent experience affects the perception of ambiguous objects. Brain research, 1322, 81-91. doi: 10.1016/j.brainres.2010.01.060

de Gelder, B., Bachoud-Levi, A. C., \& Degos, J. D. (1998). Inversion superiority in visual agnosia may be common to a variety of orientation polarised objects besides faces. Vision research, 38(18), 2855-2861.

De Jong, Ritske, Berendsen, Erna, \& Cools, Roshan. (1999). Goal neglect and inhibitory limitations: Dissociable causes of interference effects in conflict situations. Acta Psychologica, 101(2), 379-394.

De Valois, Russell L, Albrecht, Duane G, \& Thorell, Lisa G. (1982). Spatial frequency selectivity of cells in macaque visual cortex. Vision research, 22(5), 545-559.

Ekman, Gösta, Berglund, Birgitta, Berglund, Ulf, \& Lindvall, Thomas. (1967). Perceived intensity of odor as a function of time of adaptation. Scandinavian journal of psychology, 8(1), 177-186.

Gattass, R, Gross, CG, \& Sandell, JH. (1981). Visual topography of V2 in the macaque. Journal of Comparative Neurology, 201(4), 519-539.

Gattass, R., Sousa, A. P., \& Gross, C. G. (1988). Visuotopic organization and extent of $V_{3}$ and $V_{4}$ of the macaque. J Neurosci, 8(6), 1831-1845. 
Gauthier, I., Skudlarski, P., Gore, J. C., \& Anderson, A. W. (2000). Expertise for cars and birds recruits brain areas involved in face recognition. Nature neuroscience, 3(2), 191-197.

Georgeson, MA, \& Ruddock, KH. (1980). Spatial Frequency Analysis in Early Visual Processing [and Discussion]. Philosophical Transactions of the Royal Society of London. B, Biological Sciences, 290(1038), 11-22.

Ghuman, Avniel Singh, McDaniel, Jonathan R, \& Martin, Alex. (2010). Face adaptation without a face. Current biology, 20(1), 32-36.

Gibson, James J, \& Radner, Minnie. (1937). Adaptation, after-effect and contrast in the perception of tilted lines. I. Quantitative studies. Journal of Experimental Psychology, 20(5), 453.

Goffaux, V. (2009). Spatial interactions in upright and inverted faces: reexploration of spatial scale influence. Vision Res, 49(7), 774-781. doi: So042-6989(09)00053-4

Goffaux, V., Gauthier, I., \& Rossion, B. (2003). Spatial scale contribution to early visual differences between face and object processing. Brain Res Cogn Brain Res, 16(3), 416-424.

Goffaux, V., Hault, B., Michel, C., Vuong, Q. C., \& Rossion, B. (2005). The respective role of low and high spatial frequencies in supporting configural and featural processing of faces. Perception, 34(1), 77-86.

Goffaux, V., Peters, J., Haubrechts, J., Schiltz, C., Jansma, B., \& Goebel, R. (2011). From coarse to fine? Spatial and temporal dynamics of cortical face processing. Cereb Cortex, 21(2), 467-476.

Graham, Norma Van Surdam. (2001). Visual pattern analyzers: Oxford University Press.

Grill-Spector, K. (2003). The neural basis of object perception. Curr. Opin. Neurobiol., 13(2), 159-166.

Grill-Spector, K., Knouf, N., \& Kanwisher, N. (2004). The fusiform face area subserves face perception, not generic within-category identification. Nature neuroscience, 7(5), 555-562. 


\section{Chapter 3}

Grill-Spector, Kalanit, \& Malach, Rafael. (2001). fMR-adaptation: a tool for studying the functional properties of human cortical neurons. Acta Psychologica, 107(1), 293-321.

Gross, C. G., Bender, D. B., \& Rocha-Miranda, C. E. (1969). Visual receptive fields of neurons in inferotemporal cortex of the monkey. Science, 166(910), $1303-1306$.

Hadjikhani, Nouchine, Kveraga, Kestutis, Naik, Paulami, \& Ahlfors, Seppo P. (2009). Early (N170) activation of face-specific cortex by face-like objects. Neuroreport, 20(4), 403.

Hubel, D. H., \& Wiesel, T. N. (1962). Receptive fields, binocular interaction and functional architecture in the cat's visual cortex. J Physiol, 160, 106-154.

Kane, Michael J, \& Engle, Randall W. (2003). Working-memory capacity and the control of attention: the contributions of goal neglect, response competition, and task set to Stroop interference. Journal of experimental psychology: General, 132(1), 47.

Kourtzi, Zoe, \& Grill-Spector, Kalanit. (2005). fMRI adaptation: a tool for studying visual representations in the primate brain. Fitting the Mind to the World: Adaptation and After-Effects in High-Level Vision (Rhodes, G. and Clifford, CWG, eds), 173-188.

Kovacs, G., Zimmer, M., Banko, E., Harza, I., Antal, A., \& Vidnyanszky, Z. (2006). Electrophysiological correlates of visual adaptation to faces and body parts in humans. Cerebral cortex, 16(5), 742-753. doi: 10.1093/cercor/bhj020

Kovacs, G., Zimmer, M., Harza, I., Antal, A., \& Vidnyanszky, Z. (2005). Positionspecificity of facial adaptation. Neuroreport, 16(17), 1945-1949.

Kovács, Gyula, Cziraki, Csaba, Vidnyánszky, Zoltán, Schweinberger, Stefan R, \& Greenlee, Mark W. (2008). Position-specific and position-invariant face aftereffects reflect the adaptation of different cortical areas. Neuroimage, 43(1), 156-164.

Leopold, D. A., O'Toole, A. J., Vetter, T., \& Blanz, V. (2001). Prototypereferenced shape encoding revealed by high-level aftereffects. Nature neuroscience, 4(1), 89-94. doi: 10.1038/82947 
Logothetis, Nikos K. (2008). What we can do and what we cannot do with fMRI. Nature, 453(7197), 869-878.

Lueschow, A., Miller, E. K., \& Desimone, R. (1994). Inferior temporal mechanisms for invariant object recognition. Cereb Cortex, 4(5), 523531.

Lundqvist, D., Flykt, A., \& Öhman, A. (1998). The Karolinska Directed Emotional Faces-KDEF. CD-ROM from Department of Clinical Neuroscience, Psychology section, Karolinska Institutet, Stockholm, Sweden: ISBN 91630-7164-9.

Mack, M. L., Gauthier, I., Sadr, J., \& Palmeri, T. J. (2008). Object detection and basic-level categorization: sometimes you know it is there before you know what it is. Psychon Bull Rev, 15(1), 28-35.

Moscovitch, Morris, \& Radzins, Myra. (1987). Backward masking of lateralized faces by noise, pattern, and spatial frequency. Brain and Cognition, 6(1), 72-90.

Rhodes, Gillian. (1985). Lateralized processes in face recognition. British journal of Psychology, 76(2), 249-271.

Riesenhuber, M., \& Poggio, T. (1999). Hierarchical models of object recognition in cortex. Nature neuroscience, 2(11), 1019-1025. doi: 10.1038/14819

Sadr, Javid, \& Sinha, Pawan. Object recognition and Random Image Structure Evolution. Cognitive Science, 28(2), 259-287.

Sawamura, Hiromasa, Orban, Guy A., \& Vogels, Rufin. (2006). Selectivity of Neuronal Adaptation Does Not Match Response Selectivity: A SingleCell Study of the fMRI Adaptation Paradigm. Neuron, 49(2), 307-318.

Schwartz, O., Hsu, A., \& Dayan, P. (2007). Space and time in visual context. Nature reviews. Neuroscience, 8(7), 522-535. doi: 10.1038/nrn2155

Schweinberger, Stefan R, Casper, Christoph, Hauthal, Nadine, Kaufmann, Jürgen M, Kawahara, Hideki, Kloth, Nadine, . . . Zäske, Romi. (2008). Auditory adaptation in voice perception. Current biology, 18(9), 684688. 


\section{Chapter 3}

Torralba, Antonio, \& Oliva, Aude. (2003). Statistics of natural image categories. Network: computation in neural systems, 14(3), 391-412.

Webster, Michael A, Kaping, Daniel, Mizokami, Yoko, \& Duhamel, Paul. (2004). Adaptation to natural facial categories. Nature, 428(6982), 557-561.

Webster, Michael A, \& Maclin, Otto H. (1999). Figural aftereffects in the perception of faces. Psychonomic bulletin \& review, 6(4), 647-653.

Wichmann, Felix A, \& Hill, N Jeremy. (2001). The psychometric function: I. Fitting, sampling, and goodness of fit. Perception \& Psychophysics, 63(8), 1293-1313.

Xu, X., \& Biederman, I. (2014). Neural correlates of face detection. Cerebral cortex, 24(6), 1555-1564. doi: 10.1093/cercor/bhto05

Zimmer, M., \& Kovacs, G. (2011). Position specificity of adaptation-related face aftereffects. Philosophical transactions of the Royal Society of London. Series B, Biological sciences, 366(1564), 586-595. doi: 10.1098/rstb.2010.0265 


CHAPTER 4

\section{INVISIBLE FACES}

\section{Suppression of Face Perception during Saccadic Eye Movements}

Based on the article:

Mehrdad Seirafi, Peter De Weerd, and Beatrice de Gelder. "Suppression of Face Perception during Saccadic Eye Movements." Journal of ophthalmology 2014 (2014). 


\section{ABSTRACT}

Lack of awareness of a stimulus briefly presented during saccadic eye movement is known as saccadic omission. Studying the reduced visibility of visual stimuli around the time of saccade -known as saccadic suppression- is a key step to investigate saccadic omission. To date almost all studies have been focused on the reduced visibility of simple stimuli such as flashes and bars. The extension of the results from simple stimuli to more complex objects has been neglected. In two experimental tasks we measured the subjective and objective awareness of briefly presented face stimuli during saccadic eye movement. In the first task, we measured the subjective awareness of the visual stimuli and showed that in most of the trials there is no conscious awareness of the faces. In the second task, we measured objective sensitivity in a two-alternative forced choice (2AFC) face detection task, which demonstrated chance-level performance. Here, we provide the first evidence of complete suppression of complex visual stimuli during the saccadic eye movement.

Keywords: saccadic suppression, face detection, omission, mask 


\section{1) Introduction}

We typically make hundreds of saccadic eye movements every minute (Buswell, 1935; Hering, 1879), resulting in a continuous shift of the visual world on the retina. Answering the question of how stable vision is achieved during saccade has been one of the major problems in unifying models for vision.

One of the key approaches in addressing this question is to study the dynamics of visual sensitivity in a time window around the saccadic eye movement. Several lines of research have revealed a dramatic deterioration of visual sensitivity at the time of saccade, known as saccadic suppression (Burr \& Morrone, 2011; Matin, Clymer, \& Matin, 1972; Volkmann, Riggs, Moore, \& White, 1978; Zuber \& Stark, 1966). However, there is no general consensus about the domain and the underlying mechanisms of such sensitivity loss.

The source of sensitivity loss during saccade has been attributed to two different streams: top-down and bottom-up. In the top-down account, an active extra-retinal suppression process is proposed (Bridgeman, Hendry, \& Stark, 1975) which targets only the magnocellular pathway (Burr \& Ross, 1982), and does not affect the equiluminant visual stimuli (Burr, Morrone, \& Ross, 1994). On the other hand, the bottom-up account postulates a simple passive process of retinal motion smear as a source of suppression during saccade (Castet, 2010; García-Pérez \& Peli, 2011). In this account, the presence of high contrast spatial structure before and after the saccade introduces masking of the blurred perisaccadic retinal image (Campbell \& Wurtz, 1978; Castet, Jeanjean, \& Masson, 2002).

One of the questions regarding saccadic suppression is its possible effect in processing more complex and more biologically significant visual objects. The majority of saccadic eye movement studies in the past century have generally employed simple stimuli such as bars, dots, and gratings with different spatial frequencies and small window sizes that affected luminance instead of pattern detection. Some recent studies employing modern gazecontingent paradigms have been focused on vision around the time of freely made eye movements in natural dynamic scenes (Dorr \& Bex, 2013). However, such paradigms are not able to dissociate the sensitivity change during saccade from the forward- and backward masking effects introduced by the pre- and post-saccadic retinal images. Furthermore, none of these experiments have been focused on measuring the sensitivity loss during saccade for object detection or object recognition tasks. 


\section{Chapter 4}

One of the challenges of dealing with more realistic objects is how to measure the visual sensitivity in the object detection/categorization tasks. There is an ongoing debate about whether the objective or the subjective measure is appropriate for this purpose. Subjective measures are based on a subject's report about the task-relevant attribute (e.g. object category) of the stimulus. Objective measures rely on the participant's performance in a certain task regardless of the participant's conscious awareness of the task-relevant attribute. Consequently, to fill in this theoretical gap, a new approach has emerged in recent years which takes into account both subjective and objective measures (Dehaene \& Changeux, 2011; Kouider \& Dehaene, 2007; Szczepanowski \& Pessoa, 2007).

In the present study we investigated the levels of saccadic suppression for the category of complex visual objects. The goal was to see whether a salient visual stimulus could still be rendered fully invisible if presented during saccadic eye movement. This was done by measuring the sensitivity loss during saccade for face stimuli using two separate tasks: in the first task, we recorded the subjective rating of the stimuli that were presented during saccade. In the second task, we measured the participants' objective face detection performance.

\section{1) Methods and Materials}

\section{Participants}

We recruited 14 participants (11 female, $M_{\text {age }}$ : 24, age range: 18-32) from Maastricht University through local advertisements for this study. The study was conducted in accordance with university ethics committee requirements. The subjects gave informed consent to participate and were rewarded with 7.5 Euro vouchers. All participants were right-handed, with normal or corrected-tonormal vision and naive as to the purpose of the experiment. Two participants were excluded from the analysis due to the fact that they always pressed the same button to all stimuli in all conditions during the face detection task (see below).

\section{Stimuli and Procedure}

Physical setup. Movements of the right eye were measured using an EyeLink 1000 Desktop Mount (SR Research, Osgoode, Ontario, Canada) with an average spatial resolution of $0.25^{\circ}$ to $0.50^{\circ}$, sampling at $1-\mathrm{kHz}$. Manual responses 
were recorded via a standard keyboard. The real-time connection between the eye tracker and the stimulus presentation control as well as the response collection was implemented in E-Prime 2.0 Professional software (Psychological Software Tools, Inc., Pittsburgh, PA).

Image preprocessing. Original face images were color images of two males and two females, each displaying the emotional conditions of happy, fearful and neutral. These were selected from a sub-set of the Karolinska Directed Emotional Faces (KDEF) (Lundqvist, Flykt, \& Öhman, 1998), previously validated to be correctly (> 90\%) categorized as happy, fearful and neutral in a pilot study.

The stimuli were preprocessed as follows: first, all the original images were cropped and resized to fit into a rectangle matching eye position- by Adobe Photoshop CS6 (Adobe; http://adobe.com). Then, average pixel values for each image were shifted to 128 in Matlab 2012a (Mathworks; http://www.mathworks.com). Scrambled faces (scrambles) were generated by randomizing the phases of Fourier transform of the preprocessed face stimuli while keeping the Fourier power constant. The phase shuffling is quantified by a phase coherence index, in which o means all phase information has been randomized, preserving the overall distribution, and in which 100 means all phase information is intact (Goffaux et al., 2011; Mack, Gauthier, Sadr, \& Palmeri, 2008; Sadr \& Sinha, 2001). The main advantage of this technique is keeping the significant components for low-level vision (such as average luminance and Fourier power distribution) constant. The scrambles used in this experiment were generated at zero phase coherence.

Procedure and stimuli. Before the beginning of the first block, participants were familiarized with the experimental procedure using a short run of the subjective rating task (see below) consisting of 4-8 trials. After the practice phase, we showed them two target stimulus examples of the two experimental conditions in order to acquaint them with the stimuli of the scramble condition. Therefore, they were completely aware of possible face and scramble conditions.

A trial proceeded as follows: A red circle was displayed on horizontal median, $1 / 4$ of screen to the left horizontally on a gray background (Figure 1). After 3 seconds of fixation on the circle (diameter $=0.5^{\circ}$ ) as measured by the eye-tracker, a beep cued the participants to make a saccade. The auditory cue was accompanied with a saccadic target indicating where the end-location of 


\section{Chapter 4}

the saccade should be. The saccadic target was a small rectangle, $15^{\circ}$ to the right of the fixation on the horizontal meridian. As soon as the participant initiated the saccade, a task target (width $=8.5^{\circ}$ ) was displayed on top of the initial fixation point (saccadic origin) for $16.7 \mathrm{~ms}$. Next, a blank grey screen was shown for 50oms, followed by the response screen including task-relevant question (see below).

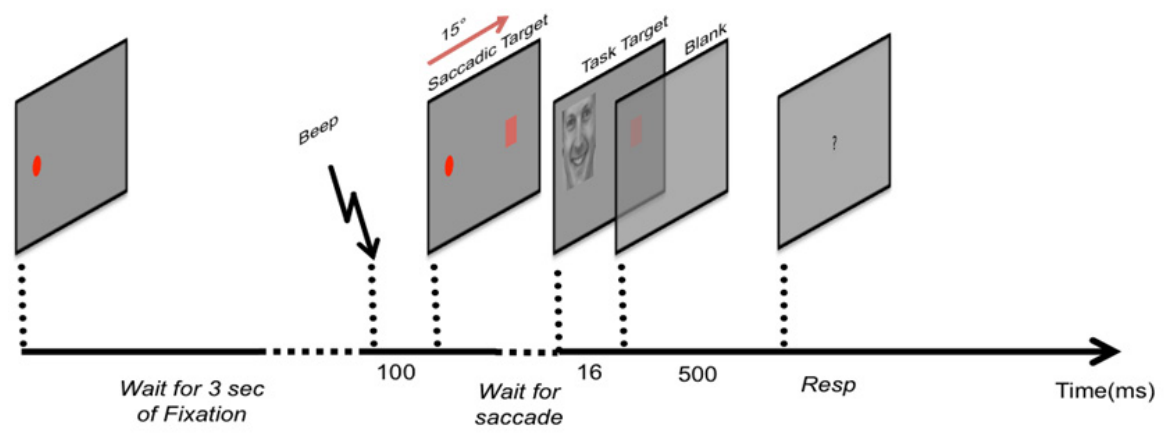

Figure 1 Schematic view of one trial: after 3 seconds, an auditory cue initiates the saccade followed by visual saccadic target. As soon as the saccade is detected, one visual stimulus (face or scramble) is presented, followed by a blank screen. Next, the task-relevant question is displayed on the screen.

Design and Tasks. The participants performed two tasks in four separate experimental blocks: The first and the last blocks were the subjective rating tasks; the second and the third blocks were the face detection task.

Task 1: Subjective rating. In the subjective rating task, the participants reported what they saw during the saccade. They were instructed to choose "nothing", "something" or "everything" by pressing the corresponding arrow keys on the keyboard: (a) left arrow key for "nothing", when they did not perceive any target stimulus or its background flashing on the screen during the saccade; (b) down arrow key for "something", when they saw a bright rectangle flashing on the screen but did not see anything inside it; (c) right arrow key for "everything", if they saw the target stimulus which could have been a face or a noisy grey texture. In addition, the participants were always asked if they had perceived something other than the three alternatives, to be sure that our options were inclusive of all the possible choices. Each experimental block in the subjective rating task consisted of 24 trials. The scramble and each of the 
three emotional face conditions (neutral, happy and fearful) had the same proportion.

Task 2: Face detection. In the face detection task, the procedure was similar to the subjective rating task except that the participants were asked to report whether they perceived the stimuli as a face or as a scramble. Left and right button presses corresponded respectively to face and scramble responses. Each experimental block in the face detection task consisted of 32 trials. In half of the trials, face stimuli (with same proportion of all three emotional conditions) and in the other half scramble stimuli were presented.

\section{Data Analysis}

Signal detection theory. The sensitivity to the signal was estimated by calculating the d-prime $\left(d^{\prime}\right)$. The $d^{\prime}$ is a measure of the distance between the signal and the noise distribution means in standard deviation units (Green \& Swets, 1966). A d' of o means that the participants are not able to discriminate the faces from scrambles in face detection task. The $d$ ' was calculated as:

$d^{\prime}=\phi^{-1}(\mathrm{H})-\phi^{-1}(\mathrm{FA})$

where $\mathrm{H}$ is the hit rate (proportion of correctly responded trials with face stimuli) and FA is the false alarm rate (proportion of correctly responded trials for scrambles). The function $\phi^{-1}$ converts the rates into $z$-scores.

Gaze analysis and saccade detection. The saccades were detected based on the commonly used saccadic threshold for the velocity of eye movement based on weighted sum of four samples:

$V^{2}+\mathrm{V}^{\prime}{ }^{2}>v(\text { thrs })^{2}$,

where $V$ and $V^{\prime}$ are components of pupil velocity on horizontal and vertical axes respectively and $v$ (thrs) is set to 40 degrees/second. Velocity for each sample was calculated by a weighted sum of four samples:

$V_{s[n]}=\left(1000\left(x_{[n+2]}+x_{[n+1]}-x_{[n-1]}-x_{[n-2]}\right)\right) /\left(6 P P D_{[n]}\right)$, 
in which the units are in true degree per second, and PPD is the corresponding resolution for each sample. In order to ensure that only the correct horizontal saccades are detected, an additional constraint was set to exclude the saccades diverging more than 5 degrees from the horizontal axis.

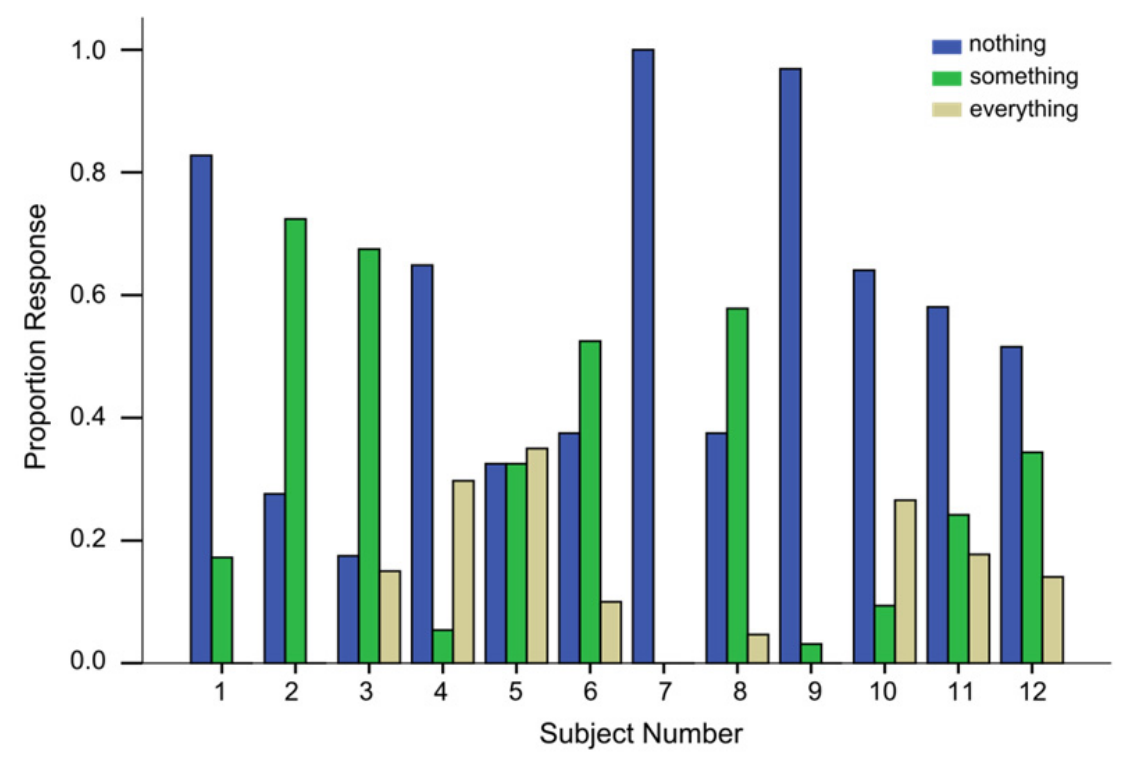

Figure 2 Proportion response in subjective rating task for every individual participant.

\section{Results}

We first pre-processed the data by removing the trials without response and the trials where no saccade was detected within a time window of 10 seconds ( $\approx 5$ percent of the whole trials). Then, we checked for any significant difference between different emotion conditions (happy, fearful and neutral) in any of the two tasks by pooling the data from all participants. For the subjective rating task, we tested if there were any significant difference between the proportions of responses to the three emotion conditions. The Pearson Chisquare non-parametric test showed no significant difference between the proportion of responses to the three emotion conditions $\left(\chi^{2}(4, N=401)=\right.$ $2.77, p=0.60)$. Afterwards, we tested if the proportion of correct responses to the three different emotional conditions differed in the face detection task. For this, we applied analysis of variance (ANOVA) with the proportion of correct response as dependent variable and emotion as independent variable. The 
average proportion of correct responses for the three emotions ( $M_{\text {Fear }}=0.43$; $\left.M_{\text {Happy }}=0.48, M_{\text {Neutral }}=0.42\right)$ did not show significant difference $(F(2,365)=$ $0.64 ; p=0.52$ ).

Subjective rating task. Figure 2 shows the proportion of responses to each of the three options in the subjective rating task. The results showed that all of the participants had no conscious awareness of the stimulus category in most of the trials ("nothing" and "something" conditions). Next, we analyzed the subjective measure of conscious perception of the stimuli in the subjective rating task averaged across the participants to see the extent of stimulus visibility during the saccade.

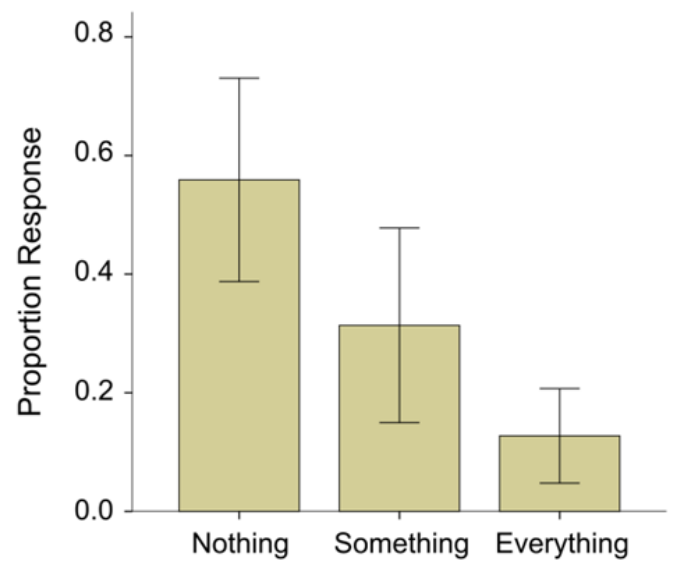

Figure 3 Proportion response to each of the three alternatives averaged across participants. The error-bars represent $95 \%$ confidence interval.

As depicted in Figure 3, the results showed that in only a small portion of all trials $(\approx 11 \%)$, could the participants consciously perceive the presented stimuli during a saccade and in about one-third of the trials the participants could only perceive a flashing square (the size of the face frame). In the remaining trials $(\approx 56 \%$ ), the participants perceived absolutely nothing during the saccade. It is noteworthy that, because of the dependence of the variables (proportion responses), analysis of variance (ANOVA) was not suitable; this might also have caused inflated significance of the correlation analysis. Thus, we compared the proportion of responses to the three response options with a non-parametric test. The Friedman test revealed a significant difference of the 


\section{Chapter 4}

three options $\left(\chi^{2}(2, N=12)=8.65, p=0.013\right)$. Then, we compared all the pairs using a non-parametric test of Wilcoxon signed ranks. The results showed a non-significant difference between "something" and "nothing" conditions ( $p$ > 0.1). The difference between "everything" and "something" was marginally significant $(Z=-1.87 ; p=0.062)$, and the difference between everything and "nothing" was significant $(Z=-2.94 ; p=0.003)$.

Face detection task. First we calculated $d$ ' as a measure of sensitivity for each participant (see Methods and Materials). Four participants exhibited below-chance behavior (negative $d^{\prime}$ ) in the face detection task. A negative $d^{\prime}$ at subject level can generally be related to either mislabeling the response options by the subject or a case of sampling error - similar to most of the studies on subliminal perception (Norman, Heywood, \& Kentridge, 2013; Szczepanowski \& Pessoa, 2007). The former is quite unlikely in our experimental setup due to presentation of response options on the screen after every single trial; hence the latter is most likely the case in this experiment. In group analysis, resetting negative $d$ 's to zero value or simply omitting them from the analysis might lead to an inflated estimate of group d' (Los Angeles Thomas, 2001; Macmillan \& Creelman, 2004), thus we kept them for the main analysis. Nevertheless, a posthoc analysis excluding only the highest negative $d^{\prime}$ (subject 1 ), exhibited no difference in the significance of the statistical results (below).

Then, we compared the $d^{\prime}$ with the baseline $\left(d^{\prime}=0\right)$ using student's ttest across all of the participants. The average $d^{\prime}$ for face detection was $0.02 \pm$ 0.075 . The results show that face detection is not significantly different from the baseline $(t(11)=0.285 ; p=0.78)$. We further analyzed the data using bootstrap for one-sample t-test with 1000 iterations. The results were consistent with the previous test showing the mean difference of 0.02 (bias: 0.002 ; standard error: 0.07; $p=0.82$ ).

Comparison of the two tasks. We, further, compared the results from the two tasks to examine the possible relationship of the proportion of responses of subjective rating from the first task and face detection sensitivity in the second task. For this, we calculated the Pearson correlation of each of the three response options in the subjective rating task with the $d^{\prime}$ calculated from the second task across the participants. The correlation of face detection sensitivity to "everything", "something" and "nothing" responses was 0.132 , $0.056,-0.11$, respectively. None of the correlations reached the significance level $(p>0.35)$. 


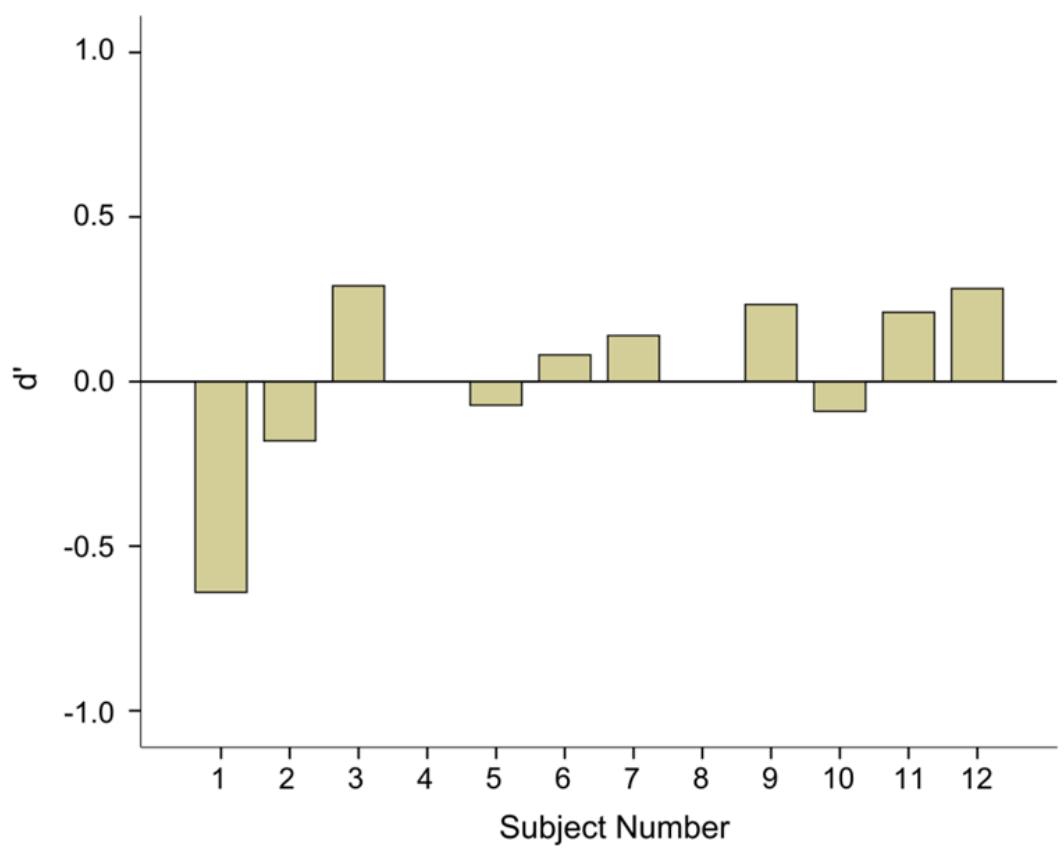

Figure 4 Sensitivity of individual participants in the face detection task.

\section{3| Discussion}

During saccadic eye movements, stable vision is maintained by the reduction of visual sensitivity. Here, we tested the extent of this reduction for complex stimuli that were briefly presented only during the saccadic eye movements. First, we showed that when a complex visual object is displayed during a saccade, the participants could not self-report its presence in $90 \%$ of trials (subjective rating task). Then, we used sensitivity analysis as an objective measure in a $2 \mathrm{AFC}$ face detection task. The results indicated near-zero discrimination sensitivity for faces versus scrambles. Finally, we examined the relationship between subjective rates from the first task and the face detection sensitivities from the second task across the participants. The results showed that none of the three rating conditions were significantly connected to the face detection performance. This suggests at least some degree of independence between the subjective and objective measures.

The results from the face detection task show that there is no awareness of the face even with objective measures during saccadic eye movement. It has been repeatedly demonstrated that the mere subjective 


\section{Chapter 4}

indication of "not seeing" the visual stimuli does not exclude the fact that certain aspects of the stimulus cannot still be processed, and be revealed by a 2AFC task (for a review see (Kouider \& Dehaene, 2007)). Thus, the combination of the two tasks is necessary. However, the validity of the "seen" trials was not directly confirmed since the two tasks were run in different experimental blocks. It is important to validate these results with simultaneous responses to both objective and subjective task in every trial.

In the current theoretical framework of object detection there is no consensus on how the conscious awareness (hence, subliminal consciousness) is defined precisely. The main debate is whether the subjective or the objective measure is suitable to measure conscious awareness. However, a third approach has emerged in the past decade arguing that both accounts are important and each one is actually representing one distinct network (Dehaene \& Changeux, 2011; Kouider \& Dehaene, 2007; Szczepanowski \& Pessoa, 2007). By introducing the two levels of "unseen" conditions in this experiment, we aimed to examine the connection of those two subjective levels to subliminal face detection performance. However, due to the chance-level performance in the face detection task and lack of simultaneous measure of the two tasks, we were unlikely to find a strong correlation only based on correlation across the participants. The absence of such strong correlation suggests that the two subjective and objective measures are independent to some extent. This is consistent with the previous research demonstrating the divergence of the two measures in certain conditions and reiterates the necessity of running both tasks in similar research (Szczepanowski \& Pessoa, 2007).

This experiment is relevant to the context of unconscious and subliminal perception from the methodological point of view. We showed that saccadic suppression is a powerful tool to mask complex visual stimuli completely. Masking has been the most important tool for studying the basic stages of visual processing. The results of this experiment show that saccadic eye movement can strongly disrupt the perception of the complex visual stimuli even if they are as salient as faces. This is interesting rendering face stimuli invisible with traditional masking techniques is a difficult task particularly when they are presented at normal contrast, binocularly, and with large sizes (Costen, Shepherd, Ellis, \& Craw, 1994; Loffler, Gordon, Wilkinson, Goren, \& Wilson, 2005). Furthermore, in forward- and backward masking paradigms both mask and target need to be displayed. To avoid the assumptions made about the interaction between the target and mask, saccadic suppression could be a useful alternative. Moreover, saccadic suppression does not need to include 
assumptions about the interaction of the different stimuli presented to each eye as in binocular rivalry where different stimuli are presented to different eyes and the percept of one eye suppresses the percept of the other eye. Hence, we believe that saccadic suppression can be employed as a complementary technique in a broad range of face/object detection and discrimination experimental designs where the visibility of the target stimulus needs to be reduced.

The current study is also relevant to the recent research revealing that certain properties of the "unseen" peri-saccadic stimuli can still be processed unconsciously and influence the perception of the following stimuli. Recently, Watson et al. (2009) showed that the unconscious processing of an oriented bar that is presented during saccade, can alter the perception of the upcoming stimulus at the end of saccade. Moreover, there is an increasing body of evidence that the suppressed face stimuli can still be processed unconsciously. Other studies have demonstrated that unperceived objects can still activate cortical (Fang \& He, 2005) and subcortical structures (Jiang \& He, 2006) under certain conditions. The results from the current study show that face detection is completely suppressed during saccade. Hence, it would be interesting to investigate if any subliminal perception can take place under these conditions. Future behavioral and imaging studies can address the possibility of such subliminal effects in the context of complex object perception. A remaining question is which underlying factors are involved in different ratings within and across the participants. For example, the timing of the stimulus presentation within the peri-saccadic interval and the speed of saccade at the time of stimulus presentation can be two relevant factors for both subjective rating and face detection tasks.

The present work is also related methodologically to the studies using "change blindness" during saccadic eye movement (Simons \& Levin, 1997). Change blindness is the inability to detect the changes to an object or scene under certain conditions. The most relevant example, which also used complex objects, is a research aiming to examine the rigidity of position-invariant object recognition (Cox, Meier, Oertelt, \& DiCarlo, 2005). In that study, the authors engineered a situation where the participants were instructed to saccade to the complex objects that were displayed peripherally. During the saccade, the identities of the peripheral objects were altered. In line with the present study, the authors first demonstrated that the participants were unaware of the identity change happening to the complex artificial target objects during saccade. Implementing this technique allowed them to construe a virtual 
environment, with different visual statistics, where the association of the peripheral and the foveal representations of certain object identities were broken. They showed that the participants who were trained in such a "position-variant" environment would lose the position-invariant object recognition drastically for the trained objects. However, that study did not disentangle the masking effect of the pre-saccadic stimulus on the postsaccadic test stimulus, since they both were presented at the same position of saccadic landing. The other methodological difference was that they used artificial objects unlike the current study.

Examining the visual system under naturalistic conditions has been one of the main goals of this work. In the past decade, the extension of the findings from simple stimuli to the real-life vision has been debated in basic vision research (Henderson, 2003). The main criticism of the opponents of using simple stimuli is that the visual system has evolved and developed to optimally analyze the visual world. However, the neurons in the early visual areas rarely receive such simple patterns as those that have been extensively used in vision research. In addition, most of these neurons mainly exhibit non-linear behavior in the presence of more complex inputs. As a result, studying vision under naturalistic conditions reveals to be an essential step to confirm the models obtained from the studies with simple stimuli. Specifically in the domain of eye movement research, there has been over a century of tradition in studying saccadic eye movements using simple visual stimuli such as light flashes, lines and Gabor patches (Ross, Morrone, Goldberg, \& Burr, 2001; Tamietto \& de Gelder, 2010). Nevertheless, the extension of such findings to the more realistic visual stimuli is questionable. Moreover, recent technological improvements enable modification of visual stimuli in real-time, depending on the viewers' gaze direction- known as gaze-contingency paradigm. Using this paradigm, it is now possible to study naturally generated saccades with real-life stimuli. For example in a recent study providing evidence against the extra-retinal source of saccadic suppression, a gaze-contingent high-definition display was developed to modify videos in retinal co-ordinates in real-time (Dorr \& Bex, 2013). The present work is one of the few examples of extending validity of saccadic suppression to natural complex objects.

\section{Acknowledgements}

This study was partly supported by Human Frontiers Science Program RGP54/2004, NWO Nederlandse Organisatie voor Wetenschappelijk Onderzoek 
400.04081, and EU FP6-NEST-COBOL043403 and FP7 TANGO to Beatrice de Gelder, and NWO VICl grant to Peter De Weerd. We also thank B. Noudoost for his thoughts on the experiment.

\section{Conflict of Interest}

The authors declare that there is no conflict of interests regarding the publication of this article. 


\section{References}

Bridgeman, Bruce, Hendry, Derek, \& Stark, Lawrence. (1975). Failure to detect displacement of the visual world during saccadic eye movements. Vision research, 15(6), 719-722.

Burr, David C, Morrone, M Concetta, \& Ross, John. (1994). Selective suppression of the magnocellular visual pathway during saccadic eye movements. Nature, 371(6497), 511-513.

Burr, David C, \& Morrone, Maria Concetta. (2011). Spatiotopic coding and remapping in humans. Philosophical Transactions of the Royal Society B: Biological Sciences, 366(1564), 504-515.

Burr, David C, \& Ross, John. (1982). Contrast sensitivity at high velocities. Vision research, 22(4), 479-484.

Buswell, Guy Thomas. (1935). How people look at pictures: University of Chicago Press Chicago.

Campbell, Fergus W, \& Wurtz, Robert H. (1978). Saccadic omission: why we do not see a grey-out during a saccadic eye movement. Vision research, 18(10), 1297-1303.

Castet, Eric. (2010). Perception of intra-saccadic motion Dynamics of Visual Motion Processing (pp. 213-238): Springer.

Castet, Eric, Jeanjean, Sébastien, \& Masson, Guillaume S. (2002). Motion perception of saccade-induced retinal translation. Proceedings of the National Academy of Sciences, 99(23), 15159-15163.

Costen, Nicholas P, Shepherd, John W, Ellis, Hadyn D, \& Craw, Ian. (1994). Masking of faces by facial and non-facial stimuli. Visual Cognition, 1(2-3), 227-251.

Cox, D. D., Meier, P., Oertelt, N., \& DiCarlo, J. J. (2005). 'Breaking' positioninvariant object recognition. Nature neuroscience, 8(9), 1145-1147. doi: $10.1038 / \mathrm{nn} 1519$ 
Dehaene, Stanislas, \& Changeux, Jean-Pierre. (2011). Experimental and theoretical approaches to conscious processing. Neuron, 70(2), 200227.

Dorr, Michael, \& Bex, Peter J. (2013). Peri-Saccadic Natural Vision. The Journal of neuroscience, 33(3), 1211-1217.

Fang, Fang, \& He, Sheng. (2005). Cortical responses to invisible objects in the human dorsal and ventral pathways. Nature neuroscience, 8(10), 13801385 .

García-Pérez, Miguel A, \& Peli, Eli. (2011). Visual contrast processing is largely unaltered during saccades. Frontiers in psychology, 2.

Goffaux, V., Peters, J., Haubrechts, J., Schiltz, C., Jansma, B., \& Goebel, R. (2011). From coarse to fine? Spatial and temporal dynamics of cortical face processing. Cereb Cortex, 21(2), 467-476.

Green, D.M., \& Swets, J.A. (1966). Signal detection theory and psychophysics. New York: Wiley.

Henderson, John M. (2003). Human gaze control during real-world scene perception. Trends in cognitive sciences, 7(11), 498-504.

Hering, E. (1879). 1942 Spatial Sense and Movements of the Eye English translation by CA Radde (Baltimore, MD: American Academy of Optometry, 1942) of "Der Raumsinn und die Bewegung des Auges". Handbuch der Physiologie.

Jiang, Yi, \& He, Sheng. (2006). Cortical responses to invisible faces: Dissociating subsystems for facial-information processing. Current biology, 16(20), 2023-2029.

Kouider, Sid, \& Dehaene, Stanislas. (2007). Levels of processing during nonconscious perception: a critical review of visual masking. Philosophical Transactions of the Royal Society B: Biological Sciences, 362(1481), 857875 .

Loffler, Gunter, Gordon, Gael E, Wilkinson, Frances, Goren, Deborah, \& Wilson, Hugh R. (2005). Configural masking of faces: Evidence for high-level interactions in face perception. Vision research, 45(17), 2287-2297. 


\section{Chapter 4}

Los Angeles Thomas, D. (2001). Elementary signal detection theory: Oxford University Press.

Lundqvist, D., Flykt, A., \& Öhman, A. (1998). The Karolinska Directed Emotional Faces-KDEF. CD-ROM from Department of Clinical Neuroscience, Psychology section, Karolinska Institutet, Stockholm, Sweden: ISBN 91630-7164-9.

Mack, M. L., Gauthier, I., Sadr, J., \& Palmeri, T. J. (2008). Object detection and basic-level categorization: sometimes you know it is there before you know what it is. Psychon Bull Rev, 15(1), 28-35.

Macmillan, Neil A, \& Creelman, C Douglas. (2004). Detection theory: A user's guide: Psychology press.

Matin, Ethel, Clymer, Arthur B., \& Matin, Leonard. (1972). Metacontrast and Saccadic Suppression. Science, 178(4057), 179-182. doi: 10.1126/science.178.4057.179

Norman, Liam J, Heywood, Charles A, \& Kentridge, Robert W. (2013). ObjectBased Attention Without Awareness. Psychological science, 24(6), 836843.

Ross, John, Morrone, M Concetta, Goldberg, Michael E, \& Burr, David C. (2001). Changes in visual perception at the time of saccades. Trends in neurosciences, 24(2), 113-121.

Sadr, Javid, \& Sinha, Pawan. Object recognition and Random Image Structure Evolution. Cognitive Science, 28(2), 259-287.

Simons, Daniel J, \& Levin, Daniel T. (1997). Change blindness. Trends in cognitive sciences, 1(7), 261-267.

Szczepanowski, Remigiusz, \& Pessoa, Luiz. (2007). Fear perception: Can objective and subjective awareness measures be dissociated? Journal of Vision, 7(4).

Tamietto, M., \& de Gelder, B. (2010). Neural bases of the non-conscious perception of emotional signals. Nature Reviews Neuroscience, 11(10), 697-709. doi: nrn2889 
Volkmann, Frances C, Riggs, Lorrin A, Moore, Robert K, \& White, Keith D. (1978). Central and peripheral determinants of saccadic suppression. Eye Movements and the Higher Psychological Functions, Lawrence Erlbaum, Hillsdale, NJ, 35-53.

Watson, Tamara L, \& Krekelberg, Bart. (2009). The relationship between saccadic suppression and perceptual stability. Current biology, 19(12), 1040-1043.

Zuber, BL, \& Stark, L. (1966). Saccadic suppression: elevation of visual threshold associated with saccadic eye movements. Experimental neurology, 16(1), 65-79. 

CHAPTER 5

\title{
FACELESS EMOTIONS
}

\author{
Emotion Categorization in the Absence of \\ Face Detection
}

Based on the article in preparation:

Mehrdad Seirafi, Peter De Weerd, and Beatrice de Gelder "Faceless Emotions: Emotion categorization in the absence of face detection". 


\section{ABSTRACT}

Detecting an object as a face is thought to be the entry point of face processing but recent evidence indicates that facial expressions can be recognized relatively independently of face categorization. We tested this hypothesis using a novel experimental paradigm whereby stimuli were rendered invisible by being presented during saccadic eye movements. The results showed that: (i) objective measures of face detection against noisy texture falls to zero if they are presented during saccadic eye movement; (ii) successful categorization of emotion can take place in the absence of face detection, suggesting a surprising degree of independence between the processes leading to face perception and emotion recognition; (iii) the metacognition performance -the ability to discriminate between one's own correct and incorrect responses- for face detection can stay above chance while the face detection performance is at chance.

The independence of emotion categorization from basic-level face detection is in sharp contrast to the hierarchical face perception models and proposes a much higher independence of representations for emotions compared to the ones for categorical face perception. This finding reflects an unprecedented differentiation of the evolutionary ancient pathways for processing emotional expressions from the ones evolved for relatively higherlevel social interactions.

Keywords: face perception, emotion recognition, saccadic suppression 


\section{1) Introduction}

Face perception and emotion recognition have been extensively studied in the past decade but their relationship is still poorly understood. The classical view is that categorization of the stimulus as a 'face' is required in order to achieve emotional expression recognition (Bruce \& Young, 1986; Haxby, Hoffman, \& Gobbini, 2000). Some studies have argued for some degree of interaction between face categorization and expression recognition (de Gelder, Frissen, Barton, \& Hadjikhani, 2003; Schweinberger \& Soukup, 1998). There is also some evidence from previous studies suggesting a relative independence between face perception and expression recognition from patient studies and the notion is supported by theories of face perception that favor a more diversified view of face perception than ventral cortex (Damasio et al., 2000; Garrido, Barnes, Sahani, \& Dolan, 2012; Tamietto \& de Gelder, 2010). But in general it has proven difficult to find a method that allows one to exclude the possibility that some aspect of the stimulus was in fact consciously detected in normal controls. One possibility to exclude the conscious perception is the line of affect blindsight research in which the patients with lesion to striate cortex guess the emotional expression despite not seeing the stimuli (Morris, DeGelder, Weiskrantz, \& Dolan, 2001). In the present study we investigated the unconscious processing of face stimuli in participants with normal vision during saccadic eye movements. Our goal was to test a possible degree of emotion processing while the face detection performance is at chance.

Saccadic suppression is a well-known phenomenon in which visual sensitivity sharply declines around the time of a saccade. The nature, the underlying mechanisms and the neural basis of saccadic suppression have been studied mostly with simple stimuli (oriented bars or Gabor patches). But very few studies have so far used it as a powerful tool for studying unconscious processes. To our knowledge there is only one study that presented complex stimuli during saccade to measure the change blindness in a study on object invariance (Cox, Meier, Oertelt, \& DiCarlo, 2005) and another study showing that faces can be rendered invisible during saccade (Seirafi, De Weerd, \& de Gelder, 2014).

We designed three experiments to assess the relationship between face detection and emotion categorization for stimuli being presented during saccades. The first experiment was an awareness task, in which the participants simply reported if they saw the stimuli or not. Experiments 2 and 3 used a 2alternative forced choice (2AFC) task (type-1), followed by a confidence rating 


\section{Chapter 5}

(type-2) task. The type-1 tasks were used to find the first order accuracies $\left(d_{1}^{\prime}{ }_{1}\right)$ in face detection (Experiment 2) and emotion categorization (Experiment 3) tasks. In the type-2 tasks, the participants reported their confidence on their 2AFC responses and were used to calculate the metacognitive performance -the ability to discriminate between one's own correct and incorrect trials- in each of the face detection and emotion categorization tasks.

\section{1| Methods and Materials}

\section{Participants}

We recruited 22 participants (18 female, Mage: 24 , age range: 18-32) from Maastricht University through local advertisements for this study. All participants performed the three experiments. The participants gave their informed consent to participate in the experiments for 7.5 Euro vouchers. All participants were right-handed, with normal or corrected-to-normal vision and naive to the purpose of the experiment. One participant was removed from the analysis due to incomplete number of blocks.

\section{Stimuli and Procedure}

The stimuli were generated from color images of two males and two females, each displaying three different emotion conditions (happy, fearful and neutral) selected from a sub-set of previously validated $(>90 \%)$ Karolinska Directed Emotional Faces (KDEF) (Lundqvist, Flykt, \& Öhman, 1998). The images were then preprocessed as follows: First, all the original images were cropped and resized to fit into an rectangle matching eye positions by Adobe Photoshop $\mathrm{CS}_{4}$ (Adobe; http://adobe.com). Next, average pixel values for each image were shifted to 128 in Matlab 2012a (Mathworks; http://www.mathworks.com). Scrambled faces (scrambles) were generated by randomizing the phases of Fourier transform of the preprocessed face stimuli while keeping the Fourier power constant. The phase shuffling is quantified by a percentage signal, in which o means all phase information has been randomized preserving the overall distribution, and in which 100 means all phase information is intact. This method is similar to a previously established technique called Random Image Structure Evolution (RISE), used in similar experiments (Goffaux et al., 2011; Mack, Gauthier, Sadr, \& Palmeri, 2008; Sadr \& Sinha). The main advantage of this technique is keeping the average intensity and frequency distribution of the 
image constant. Such attributes have been shown to heavily affect the low-level processing of the image. In the scramble conditions, we used the images with 0 phase coherence (completely unrecognizable as face).

The participants performed the three experiments (subjective rating, face detection and emotion categorization) one after the other in three separate experimental blocks. The first and the last blocks were always the subjective rating task; the order of the other two tasks was counterbalanced among the subjects

As depicted in Figure $1 \mathrm{~A}$, a trial proceeded as follows: A red circle was presented on the horizontal median, $1 / 4$ of screen to the left horizontally. After 3 seconds of fixation within $2^{\circ}$ of the fixation point measured by the eye-tracker, a beep cued the participants to make a saccade towards an appearing rectangle 15 degree apart from the stimulus. The diameter of the fixation circle and the side of saccadic target were $0.75^{\circ}$. As soon as the participant started making the saccade, a target stimulus was presented on above the fixation circle for 16.7 ms. Then, a blank grey screen was shown for $500 \mathrm{~ms}$, followed by the response screen including the question regarding the experiment task. Trials were presented in a pseudo-random order. Prior to the first block, the instructions were presented on the screen and the subjects were trained for 2 minutes to learn the button associations and get familiar with the stimuli. When training was over, the instructions were displayed again and the experimental block started with the subject's button press. At the end of the practice, we showed participants two examples of target stimuli of every experimental condition. This was important to familiarize them with the noisy (scrambled) textures in the no-face condition.

The subjective rating task (Experiment 1) consisted of 2 blocks each of which contained 32 trials (8 of each condition: happy, fearful, neutral, scramble). In the face detection task, a block consisted of 6 cycles of each trial in which either one of the 6 noisy textures or 6 face images ( 3 of each emotional conditions of happy, fearful and neutral) was shown for $16.7 \mathrm{~ms}$ [72 Samples (6 cycles x 12 samples/cycle; No Repeat After Reset)]. In the emotion categorization task, one block consisted of 6 cycles of each trial in which either one of the 6 noisy textures or 6 face instance images ( 2 of each emotional conditions of happy, fearful and neutral) was shown for 16.7 ms [120 Samples (6 cycles x 20 samples/cycle; No Repeat After Reset)]. 


\section{Chapter 5}

A
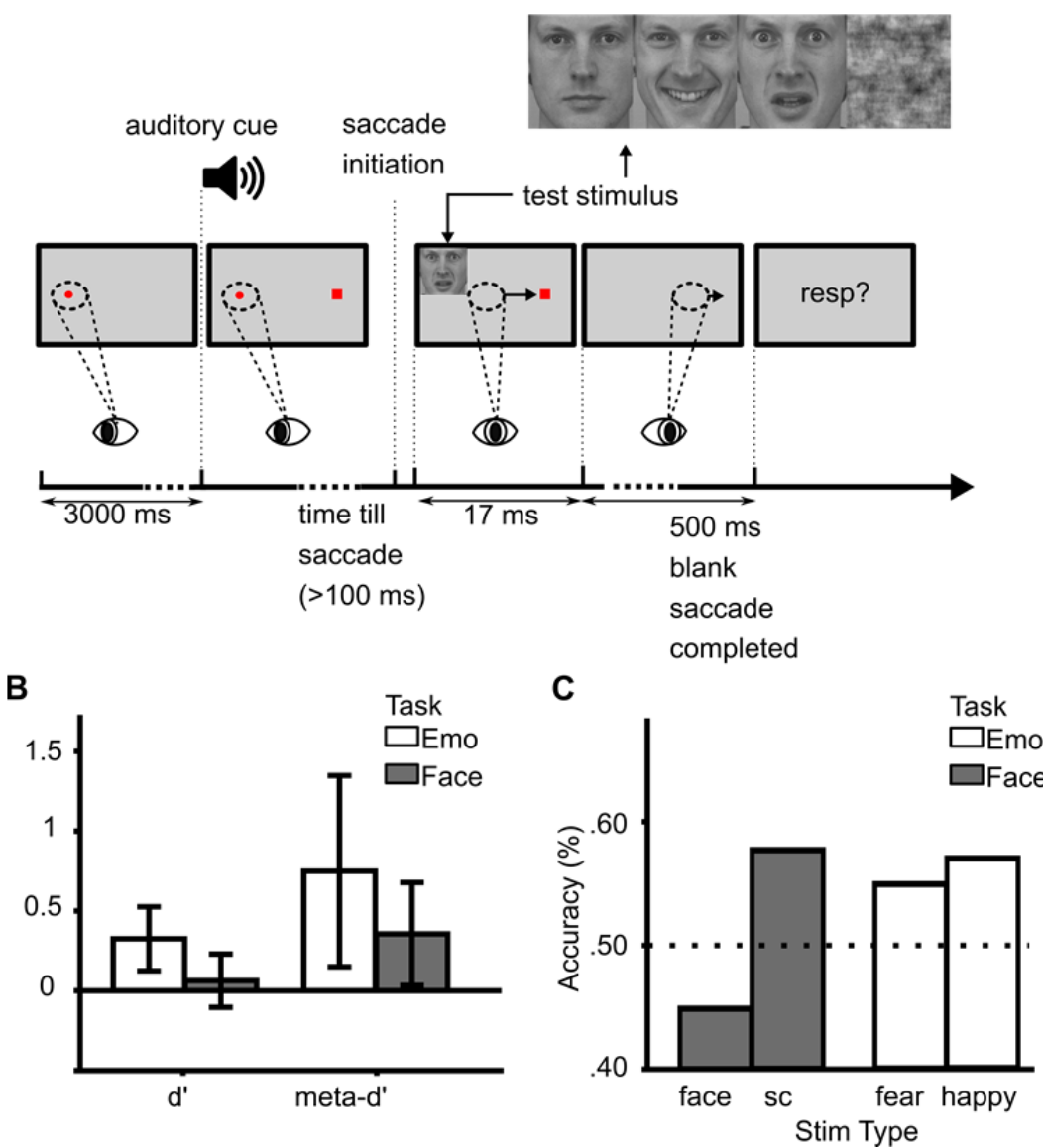

Figure 1 Experimental Design and the Results from Experiments 1-3 (A) Schematic view of one trial and sample test stimuli: A trial started with a fixation for $\mathbf{3}$ seconds at a red circle control by an eye tracker. Then, an auditory cue was executed followed by a saccadic target (red square) after $100 \mathrm{ms,}$ $15^{\circ}$ apart from the fixation. After saccade was initiated towards the red square the eye tracker waited for a right saccade. As soon as the onset of a correct saccade was detected by the eye tracker, the target stimulus was briefly presented (on the first refreshed frame) above the location of the saccadic departure for $17 \mathrm{~ms}$, followed by a blank screen ( $500 \mathrm{~ms}$ ). Next, the participant was required to press the task-relevant response. The target stimuli in Experiment 1 (awareness report: seen vs not seen) consisted of four conditions (happy, fearful, neutral, scramble). In Experiment 2 (face detection: face vs no-face), the test stimuli consisted of 3 conditions (fear, happy, scramble). Experiment 3 (emotion categorization: fear vs happy), included two emotion conditions (happy, fear). (B) Cognitive and metacognitive performance in face detection and emotion discrimination tasks (experiments 2 and 3): the white and the grey bars represent the emotion categorization and the face detection tasks respectively. The left side is the comparison of the first-order accuracy and $\left(d^{\prime}\right)$ and the right side is the comparison of the metacognition measure (meta- $\left.d^{\prime}\right)$. The error bars represent $95 \%$ confidence interval. (C) Percentage accuracy for each of the stimulus conditions in face detection and emotion categorization tasks. 


\section{Data Analysis}

Signal Detection analysis. The sensitivity to the signal was estimated by calculating the d-prime ( $\left.d^{\prime}\right)$. The $d^{\prime}$ is a measure of the distance between the signal and the noise distribution means in standard deviation units (Green \& Swets, 1966). A d' of o means that the participants are not able to discriminate the faces from scrambles in the face detection task. The $d$ ' was calculated as:

$d^{\prime}=\phi^{-1}(H)-\phi^{-1}(F A)$,

where $H$ is the hit rate (proportion of correctly responded trials with face stimuli) and $F A$ is the false alarm rate (proportion of correctly responded trials for scrambles). The function $\phi^{-1}$ converts the rates into $z$-scores. Since we were not sure about the equality of the variances of the two underlying distributions, the $d$ ' was calculated without the assumption of the equality of the variances.

Metacognition and Meta-d'. Metacognition is generally defined as the cognition about the cognition. More specifically in terms of a visual recognition task, it refers to the subjects' awareness about the correctness of their responses and used to evaluate one's ability to distinguish between correct and incorrect responses. Meta-d' is a concept closely linked to the first order accuracy ( $\left.d^{\prime}\right)$, relating the response of the type-2 task (sure, not sure) to the accuracy of the responses. In other words, the better the subject can distinguish between his/her correct and incorrect answers, the higher meta- $d^{\prime}$ is. We calculated the meta-d' using the TYPE2_SDT toolbox under Matlab (Maniscalco \& Lau, 2014). The calculations were made without the assumption of equality of variances since we were not sure about the underlying distributions.

Eye tracking. Movements of the right eye were measured using an EyeLink 1000 Desktop Mount (SR Research, Ontario, Canada) with an average spatial resolution of 0.25 - to $0.5-$, sampling at $1-\mathrm{kHz}$. Manual responses were recorded via a standard keyboard. The experimental software controlling stimulus display and response collection was implemented in Stimuli were presented using EPrime 2.0 Professional software (Psychological Software Tools, Inc., Pittsburgh, PA). 


\section{Chapter 5}

Gaze analysis and saccade detection. The saccades were detected based on the commonly used saccadic threshold for the velocity of eye movement based on weighted sum of four samples:

$V^{2}+V^{\prime 2}>v(t h r s)^{2}$,

where $\mathrm{V}$ and $\mathrm{V}^{\prime}$ are components of pupil velocity on horizontal and vertical axes respectively and $v$ (thrs) is set to 40 degrees/second. Velocity for each sample was calculated by a weighted sum of four samples:

$V s[n]=(1000(x[n+2]+x[n+1]-x[n-1]-x[n-2])) /(6 P P D[n])$,

in which the units are in true degree per second, and PPD is the corresponding resolution for each sample. In order to ensure that only the correct horizontal saccades are detected, an additional constraint was set to exclude the saccades diverging more than 5 degrees from the horizontal axis.

\section{$2 \mid \quad$ Results}

First, we preprocessed the data by removing the trials in which no saccade was performed ( $<1 \%$ of the entire data). Then we analyzed the data from the subjective rating task (Experiment 1 ) in which the participants only reported whether or not they saw the stimuli in each trial. The results showed that on average all face stimuli had the same visibility. The happy, fearful and neutral faces were rated as 'seen' in $10.2 \%, 10.2 \%$ and $11.3 \%$ of trials. However, the occurrence of reporting scrambled images as seen was half of that of the faces $5.3 \%$. This could be due to the bias for meaningful object to be rated as seen more often. These results are in line with our previous research showing $90 \%$ of face images were not seen during saccadic eye movement (Seirafi et al., 2014).

In Experiment 2 and 3, the participants performed face detection and emotion categorization tasks, respectively, and for each trial rated their confidence about their response. The data from the neutral condition in the face detection task were not included in the further analysis to keep the face stimulus set the same as in the emotion categorization task. We calculated the average $d^{\prime}$ and meta $d^{\prime}$ for each task over all participants. As shown in Figure 1B, the average $d^{\prime}$ for the face detection task and emotion categorization task were $0.06 \pm 0.07$ and $0.33 \pm 0.09$ respectively. The average meta-d' of the face 
detection task and the emotion categorization tasks were $0.35 \pm 0.15$ and $0.74 \pm$ 0.28 . Then, we compared each of the cognition and metacognition measures against zero ( $d^{\prime}=0$; meta- $d^{\prime}=0$ ) using one sample t-tests. The results showed that except for the $d^{\prime}$ in the face detection task, which was not significant ( $t$ $\left.(20)=0.8, p_{2 \text {-tail }}=0.4\right)$, all the other three measures were significantly larger than zero ( $d_{\text {emo: }}^{\prime} \mathrm{t}(20)=3.4, \mathrm{p}=0.003$; meta- $d^{\prime}{ }_{\text {face: }} \mathrm{t}(20)=2.3, p_{2 \text {-tail }}=0.032$; meta- $\left.d_{\text {emo }}: t(20)=2.6, p_{2 \text {-tail }}=0.017\right)$. Furthermore, we compared each of the two detection threshold ( $\left.d^{\prime}\right)$ and metacognition measure (meta- $d^{\prime}$ ) across different tasks using univariate analysis of variance (ANOVA). As presented in Figure $1 C$, the results revealed that the emotion categorization threshold $\left(d_{\text {emo }}^{\prime}\right)$ is significantly larger than that of the face detection task ( $d_{\text {face }}^{\prime}$ ) (mean $=0.26, \mathrm{t}$ $\left.(20)=2.62 ; p_{2 \text {-tail }}=0.016\right)$. Furthermore, we also applied paired sample t-test to the metacognition measures. This did not reveal a significant difference between the metacognition of the two tasks ( mean $=0.39, \mathrm{t}(20)=1.2 ; p_{\text {-tail }}=$ 0.2 ).

One confounding factor in the observed effect (face detection at chance while emotion categorization significantly higher than chance) could be the difference in the stimulus set used in the face detection task compared to the emotion categorization task. As described before, the face detection task consisted of both faces and scrambled stimuli whereas the emotion categorization task consisted of faces only. One could hypothesize that the observed low face detection d' in the face detection task might be due to the lower saliency of the scrambled stimuli (suggested by lower subjective rating) in the face detection task. In other words the lower $d$ ' in the face detection task might be due to smaller amount of correct rejections (correctly rejecting scrambled stimuli as a face) and not less hit rates (correctly categorizing face stimuli as face). To address this, we examined the accuracy for each of the stimulus conditions in the face detection and emotion tasks separately to test whether the scrambled images were less accurately rejected as face across all participants. The results showed an opposite trend: face detection accuracy for face stimuli was $45 \% \pm 5 \%$, whereas the accuracy of scrambled images was $58 \% \pm$ $5 \%$. This rules out the observed emotion categorization performance in the absence of face detection to be caused by the subjective rating -thus visibility difference- of the face and scrambles.

Finally, we investigated the relationship between the subjective awareness measure and the metacognition measures from the two tasks. For this, we correlated the percentage seen results from Experiment 1 with meta-d' measures across participants in both face detection and emotion categorization 


\section{Chapter 5}

tasks (derived from Experiment 1). Interestingly, the subjective awareness highly correlated with the emotion categorization metacognition $(N=21$, Pearson $r^{2}=0.65, p_{2 \text {-tail }}=0.002$ (corrected), but not with the face detection metacognition $\left(N=21\right.$, Pearson $\left.r^{2}=0.14, p_{2-\text { tail }}=0.56\right)$.

\section{3| Discussion}

In a series of three experiments we investigated the extent of residual visual abilities when normal vision was suppressed due to a saccadic eye movement. Experiment 1 examined the subjective visibility of stimuli presented during saccades. The results showed that about $90 \%$ of stimuli are not seen if they are presented during a saccade. Furthermore, face detection task (experiments 2) demonstrated that this visibility is not enough for the participants to reach a significant face detection performance. However, using the same set of face stimuli (happy and fearful) in the emotion categorization task revealed an interesting phenomenon of successful emotion categorization despite poor face detection. Another remarkable finding of this study is that the metacognition ability is already exceeding the chance even for the face detection task. Finally, the subjective awareness measure and metacognition highly correlate in the emotion categorization task but not in the face detection task.

During saccadic eye movements our perception is suppressed to a large extent- a phenomenon known as saccadic suppression. We recently proposed using saccadic suppression as a means to investigate unconscious perception (Seirafi et al., 2014). Suppression of simple stimuli (like oriented bars, or light flashes) being presented during saccades has long been described (Dorr \& Bex, 2013). However, the extent of such effect for more complex and biologically plausible stimuli has not been fully investigated. The only other research that took advantage of this phenomenon for complex stimuli is a study by Cox et al. where they showed that the participants would not successfully detect the changes to the complex stimuli that happen during saccade (Cox et al., 2005).

The results from subjective rating tasks indicate that face stimuli can be completely masked during saccadic eye movements is in line with our previous research (Seirafi et al., 2014) and is consistent with previous studies showing a decline of visual sensitivity during saccades for simple stimuli-such as oriented bars, Gabor patches and flash lights (Ross, Morrone, Goldberg, \& Burr, 2001). The present finding is also in line with a previous study (Cox et al., 2005) 
showing insensitivity of the viewers to changes to complex objects during saccade (change blindness).

Although most studies measuring type-1 and type-2 performances, show higher $d$ ' than the meta-d', our results exhibited a different pattern. We compared the two measures for each task showing that the meta-d' values were higher in both tasks compared to the d's. This is similar to the findings of Charles, Van Opstal, Marti, and Dehaene (2013) in a speeded error detection task, although here the metacognitive ability diminished as they removed the time pressure in the type1 task. However, we demonstrated the existence of such behavior in the absence of speeded task. Another similar finding to the present metacognitive results is a recent work in field of artificial language by Scott, Dienes, Barrett, Bor, and Seth (2014). They called the absence of first order accuracy in the presence of metacognition ability "blind insight". Although their effect seems similar to the findings of the present study, we have serious concerns about their subsampling methodology which might undermine their findings (Seirafi et al., submitted).

The present study opens a window to generally less-accepted models for face recognition, namely the non-hierarchical models. All present hierarchical models for face perception, take face detection as an entry point for the perception of facial attributes (emotional expression, gender, etc.). Traditional models of face perception are built on the notion that processing involves two sequentially organized stages, one of category assignment and the other of affective valence attribution. The newer models postulates two parallel pathways, one of which is devoted to rapid extraction of relevant information before detailed processing of the visual properties (Anderson, Christoff, Panitz, De Rosa, \& Gabrieli, 2003; Bar et al., 2001; de Gelder \& Rouw, 2001; Garrido et al., 2012; Rudrauf et al., 2008; Vuilleumier, 2005). In this account, emotion categorization uses a coarse representation of faces that might not be sufficient for face identification, but that might still permit successful emotion categorization.

Furthermore, a recent study using faces (Fahrenfort et al., 2012) showed that visible and invisible faces produced similar category-selective responses in the ventral visual cortex in a series of functional magnetic resonance imaging ( $\mathrm{fMRI}$ ) and electroencephalography (EEG) experiments. The pattern of neural activity evoked by visible faces could be used to decode the presence of invisible faces and vice versa. But only visible faces caused extensive response enhancements and changes in neural oscillatory synchronization, as well as increased functional connectivity between higher 


\section{Chapter 5}

and lower visual areas. They concluded that conscious face perception is more tightly linked to neural processes of sustained information integration and binding than to processes accommodating face category tuning. Their results suggest that faces that are not consciously perceived can still evoke neural activity in the face selective areas but not induce the neural synchrony to the other areas for the conscious perception. In the framework of our study, their findings suggest that the face code is available in the ventral stream but not accessible for the conscious face detection system. On the other hand, our results show that such face codes can still be accessed by the emotion categorization task. Moreover, we found that such face codes are available for both face detection and emotion categorization metacognitive tasks.

The existence of different entry representations and possibly different types of information for different facial information attributes may be tested in future experiments by assessing the effect of structural facial transformations on face and object categorization. A better understanding of how our visual system extracts emotions from a face is theoretically important, and we therefore believe our findings can give useful input to both computational modelling efforts and neurophysiological investigations of emotion and face perception.

In summary, we found that emotion categorization can take place in the absence of face detection. We also demonstrated for the first time in a visual task that metacognition can take place in the absence of first-order accuracy. We furthermore verified that such a difference is specific to the face stimuli and not dependent on the other distractor stimuli to which the faces were contrasted to. A better understanding of how our visual system extracts emotions from a face is theoretically important, and we therefore believe our findings can give useful input to both computational modeling efforts and neurophysiological investigations of emotion and face perception.

\section{Acknowledgements}

This study was partly supported by Human Frontiers Science Program RGP54/2004, NWO Nederlandse Organisatie voor Wetenschappelijk Onderzoek 400.04081, and EU FP6-NEST-COBOL043403 and FP7 TANGO to Beatrice de Gelder, and NWO VICl grant to Peter De Weerd. We also thank B. Noudoost for his thoughts on the experiment. 


\section{Conflict of Interest}

The authors declare that there is no conflict of interests regarding the publication of this article. 


\section{References}

Anderson, A. K., Christoff, K., Panitz, D., De Rosa, E., \& Gabrieli, J. D. (2003). Neural correlates of the automatic processing of threat facial signals. $J$ Neurosci, 23(13), 5627-5633.

Bar, M., Tootell, R. B., Schacter, D. L., Greve, D. N., Fischl, B., Mendola, J. D., . . . Dale, A. M. (2001). Cortical mechanisms specific to explicit visual object recognition. Neuron, 29(2), 529-535.

Bruce, V., \& Young, A. (1986). Understanding face recognition. Br J Psychol, 77 (Pt 3), 305-327.

Charles, Lucie, Van Opstal, Filip, Marti, Sébastien, \& Dehaene, Stanislas. (2013). Distinct brain mechanisms for conscious versus subliminal error detection. Neuroimage, 73, 80-94.

Cox, D. D., Meier, P., Oertelt, N., \& DiCarlo, J. J. (2005). 'Breaking' positioninvariant object recognition. Nature neuroscience, 8(9), 1145-1147. doi: $10.1038 / \mathrm{nn} 1519$

Damasio, A. R., Grabowski, T. J., Bechara, A., Damasio, H., Ponto, L. L., Parvizi, J., \& Hichwa, R. D. (2000). Subcortical and cortical brain activity during the feeling of self-generated emotions. Nature neuroscience, 3(10), 1049-1056.

de Gelder, B., Frissen, I., Barton, J., \& Hadjikhani, N. (2003). A modulatory role for facial expressions in prosopagnosia. Proceedings of the National Academy of Sciences of the United States of America, 100(22), 1310513110. doi: 10.1073/pnas.1735530100

de Gelder, B., \& Rouw, R. (2001). Beyond localisation: a dynamical dual route account of face recognition. Acta Psychol (Amst), 107(1-3), 183-207.

Dorr, Michael, \& Bex, Peter J. (2013). Peri-Saccadic Natural Vision. The Journal of neuroscience, 33(3), 1211-1217.

Fahrenfort, Johannes J, Snijders, Tineke M, Heinen, Klaartje, van Gaal, Simon, Scholte, H Steven, \& Lamme, Victor AF. (2012). Neuronal integration in visual cortex elevates face category tuning to conscious face perception. Proceedings of the National Academy of Sciences, 109(52), 21504-21509. 
Garrido, M. I., Barnes, G. R., Sahani, M., \& Dolan, R. J. (2012). Functional evidence for a dual route to amygdala. Curr Biol, 22(2), 129-134. doi: Sog60-9822(11)01371-6

Goffaux, V., Peters, J., Haubrechts, J., Schiltz, C., Jansma, B., \& Goebel, R. (2011). From coarse to fine? Spatial and temporal dynamics of cortical face processing. Cereb Cortex, 21(2), 467-476.

Green, D.M., \& Swets, J.A. (1966). Signal detection theory and psychophysics. New York: Wiley.

Haxby, J. V., Hoffman, E. A., \& Gobbini, M. I. (2000). The distributed human neural system for face perception. Trends in cognitive sciences, 4(6), 223-233.

Lundqvist, D., Flykt, A., \& Öhman, A. (1998). The Karolinska Directed Emotional Faces-KDEF. CD-ROM from Department of Clinical Neuroscience, Psychology section, Karolinska Institutet, Stockholm, Sweden: ISBN 91630-7164-9.

Mack, M. L., Gauthier, I., Sadr, J., \& Palmeri, T. J. (2008). Object detection and basic-level categorization: sometimes you know it is there before you know what it is. Psychon Bull Rev, 15(1), 28-35.

Maniscalco, Brian, \& Lau, Hakwan. (2014). Signal Detection Theory Analysis of Type 1 and Type 2 Data: Meta-d', Response-Specific Meta-d', and the Unequal Variance SDT Model The Cognitive Neuroscience of Metacognition (pp. 25-66): Springer.

Morris, J. S., DeGelder, B., Weiskrantz, L., \& Dolan, R. J. (2001). Differential extrageniculostriate and amygdala responses to presentation of emotional faces in a cortically blind field. Brain, 124(Pt 6), 1241-1252.

Ross, John, Morrone, M Concetta, Goldberg, Michael E, \& Burr, David C. (2001). Changes in visual perception at the time of saccades. Trends in neurosciences, 24(2), 113-121.

Rudrauf, D., David, O., Lachaux, J. P., Kovach, C. K., Martinerie, J., Renault, B., \& Damasio, A. (2008). Rapid interactions between the ventral visual stream and emotion-related structures rely on a two-pathway architecture. J Neurosci, 28(11), 2793-2803. doi: 28/11/2793 


\section{Chapter 5}

Sadr, Javid, \& Sinha, Pawan. Object recognition and Random Image Structure Evolution. Cognitive Science, 28(2), 259-287.

Schweinberger, Stefan R, \& Soukup, G Rüdiger. (1998). Asymmetric relationships among perceptions of facial identity, emotion, and facial speech. Journal of Experimental Psychology: Human perception and performance, 24(6), 1748.

Scott, Ryan B, Dienes, Zoltan, Barrett, Adam B, Bor, Daniel, \& Seth, Anil K. (2014). Blind Insight Metacognitive Discrimination Despite Chance Task Performance. Psychological science, 0956797614553944.

Seirafi, Mehrdad, Valente, Giancarlo, De Weerd, Peter, \& de Gelder, Beatrice. Blind sight and blind insight: How small sample sizes can affect metacognition analysis. Submitted

Seirafi, Mehrdad, De Weerd, Peter, \& de Gelder, Beatrice. (2014). Suppression of Face Perception during Saccadic Eye Movements. Journal of ophthalmology, 2014.

Tamietto, M., \& de Gelder, B. (2010). Neural bases of the non-conscious perception of emotional signals. Nature Reviews Neuroscience, 11(10), 697-709. doi: nrn2889

Vuilleumier, P. (2005). Cognitive science: staring fear in the face. Nature, 433(7021), 22-23. doi: 10.1038/433022a 


CHAPTER 6

\section{AUDIOVISUAL BLINDSIGHT}

Audiovisual Learning in the Absence of
Primary Visual Cortex

Based on the submitted article:

Seirafi, Mehrdad, Peter De Weerd, Alain Pegna, and Beatrice de Gelder. "Audiovisual association learning in the absence of primary visual cortex". 


\section{ABSTRACT}

Learning audiovisual associations is mediated by the primary cortical areas; however, recent animal studies suggest that such learning can take place even in the absence of the primary visual cortex. Other studies have demonstrated the involvement of extra-geniculate pathways and especially the superior colliculus (SC) in audiovisual association learning. Here, we investigated such learning in a rare human patient with complete loss of the bilateral striate cortex. We carried out an implicit audiovisual association learning task with two different colors of red and purple (the latter color known to minimally activate the extra-genicular pathway). Interestingly, the patient learned the association between an auditory cue and a visual stimulus only when the unseen visual stimulus was red, but not when it was purple. The current study is the first evidence showing the possibility of audiovisual association learning in humans with lesioned striate cortex. Furthermore, in line with animal studies, it supports an important role for the SC in audiovisual associative learning.

Keywords: audiovisual learning, superior colliculus, blindsight 


\section{Introduction}

There is strong evidence from neurophysiological animal experiments for the confluence of visual and auditory cues in superior colliculus (SC) (Alvarado, Vaughan, Stanford, \& Stein, 2007; Jay \& Sparks, 1984; Meredith \& Stein, 1986; Yu, Rowland, \& Stein, 2010). This suggests a major role in audiovisual association-based learning. To test this idea, the role of the geniculo-cortical pathway can be limited (thus increasing the role of the extra-genicular pathway) by ablating the striate cortex in animals. This has been done in the cat (McHaffie, Jiang, Stein, \& Willner, 2012), but due to the strong retino-genicular projections to extrastriate cortex (Rosenquist, 1985), it is difficult in such experiments to attribute audiovisual learning to extra-genicular pathways and, specifically, the SC. In monkeys, lateral geniculate nucleus (LGN) targets the primary visual cortex (V1) almost exclusively (Sincich \& Horton, 2005), such that a $\mathrm{V} 1$ lesion will render the animal blind, and make any residual visual capacities (Humphrey, 1974) and capacities for audiovisual learning, dependent on extragenicular pathways and nuclei, including the SC.

A rare patient $(\mathrm{TN})$ with a complete bilateral lesion of striate cortex gave us the opportunity to study potential contributions of the SC to human audiovisual learning in the absence of the retino-geniculo-cortical pathway. Establishing audiovisual learning in hemianopic patients and understanding the underlying mechanisms is important in view of the potential for audiovisual learning in such patients which can improve self-reliance.

The indication that there is sufficient visual information being processed that could be associated with normally perceived auditory information led to the idea to study audiovisual learning in an individual with a striate cortex lesion. Although damage to striate cortex severally disrupts normal visual processes causing blindness (i.e., the absence of conscious visual perception), some patients show remarkable residual visual abilities as evidenced by above-chance detection or discrimination of movement (Barbur, Ruddock, \& Waterfield, 1980), wavelength (Stoerig \& Cowey, 1989), flicker (Blythe, Kennard, \& Ruddock, 1987), orientation (Weiskrantz, 1974), motion direction (Pizzamiglio, Antonucci, \& Francia, 1984), and object shape (Weiskrantz, 1974). The extra-genicular retino-cortical pathway is a possible substrate underlying residual, visually-driven behavior (Weiskrantz, 1974). The SC is the earliest post-retinal structure of this pathway, and remarkably, does not receive direct input from the s-cones (Leh, Mullen, \& Ptito, 2006; Marzi, Mancini, Metitieri, \& Savazzi, 2009; Schiller \& Malpeli, 1977; Tamietto et al., 


\section{Chapter 6}

2010). Because of the important role of the SC in audiovisual integration (Meredith \& Stein, 1986), we hypothesized a contribution of SC in audiovisual association learning, which we believe to be exclusive for colored stimuli in medium-to-long wavelengths. These ideas led us to test two predictions in a new implicit audiovisual association task, in which some auditory target stimuli were predictable on the basis of associated unseen visual information. We predicted first, that given the audio-visual integrative function of the SC, the cortically blind patient TN would acquire shortened reaction times to the visually predicted auditory targets, and, second, given the SC's lack of sensitivity for short wavelengths (Marzi et al., 2009), that this reaction time advantage would be absent for visual stimuli that were colored purple.

\section{1) Materials and Methods}

\section{Participant}

The participant was a 56 year-old right-handed patient (TN) who became cortically blind bilaterally following two strokes within six weeks (Figure 1A). Previous studies documented his inability to perceive color, movement, and object shape presented in the visual modality alone, yet, he demonstrated above-chance level performance in a facial expression discrimination task (Pegna, Khateb, Lazeyras, \& Seghier, 2005) and intact visual navigation skill (de Gelder et al., 2008). The participant gave informed consent in accordance with the Helsinki protocols, and all procedures were performed according to requirements of the Ethical Committee of the Faculty of Psychology and Neuroscience.

\section{Experimental Design and Procedure}

TN was presented with an auditory looming stimulus, smoothly increasing in volume over a period of one second (symbolized in green in Figure 1B). In a subset of trials a brief 'blip' was clearly audible during the trial, occurring towards the middle (target trials, bottom Figure 1B), whereas in other trials this 'blip' was absent (non-target trials). The patient's task was to press a button as quickly as possible upon hearing the auditory target. One third of the trials consisted of target trials, and two thirds were non-target trials. A single experiment comprised 270 one-second-stimulus presentations, followed by a 
500ms response window, and an inter-trial interval lasting between 400 and $700 \mathrm{~ms}$.

A

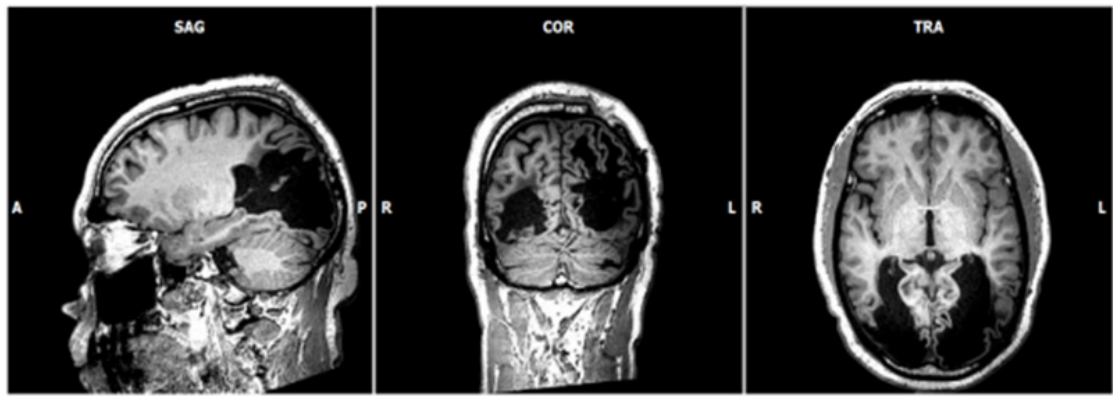

B

Percentage

Percentage

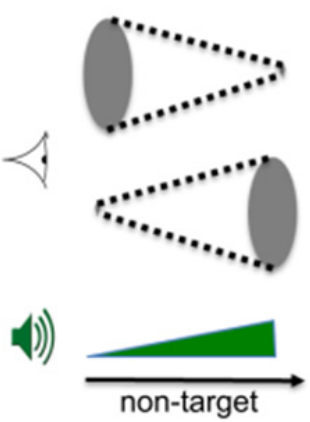

$33.5 \%$

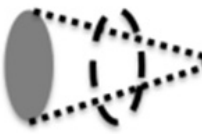

$22 \%$

$33.5 \%$

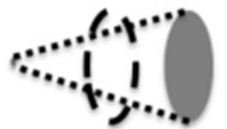

$11 \%$

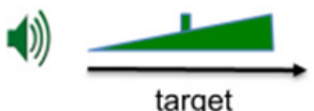

target

Figure 1 (A) T1-weighted MRI (axial, coronal and saggital views) showing the extent of bilateral striate cortex lesions. In terms of Brodmann's areas, the left hemisphere lesion encompasses BA 17, 18, 19, 37 and 39. The right hemisphere lesion encompasses BA 17, 18 and 19. Lesion sites look black as they are filled with CSF (Cerebrospinal fluid). (B) Schematic view of the target and non-target combination of audiovisual stimuli, with numbers indicating relative ratios of the different stimulus types.

In Experiment 1, we created an audio-visual association which was predictive of the presence of the auditory target. Two red visual disk stimuli were used (symbolized by grey ellipses in Figure 1B), increasing in size (looming) or decreasing (receding). To ensure visual stimulation, we used a small viewing distance $(\sim 25 \mathrm{~cm})$ and gaze was monitored by two of the experimenters. The patient was not given information about the presence of any systematic associations between auditory and visual stimuli, but was informed that visual stimuli were being presented. The audiovisual pairings were designed as 


\section{Chapter 6}

follows: In non-target trials $(\mathrm{n}=180)$, half of the trials were associated with simultaneously receding visual stimuli, and the other half were associated with looming visual stimuli. However, among auditory target trials $(n=90)$, two-thirds of the trials $(n=60)$ paired the auditory stimulus with a receding visual signal, and only one-third $(n=30)$ paired the auditory stimulus with a looming visual signal. Therefore, in the overall distribution of audio-visual pairings, the presence of a receding visual stimulus is predictive of an auditory target (visually cued target). To maintain the parallelism of visual and auditory stimuli, we included a visual target analogue in the visually looming or receding stimuli, paired with auditory targets. The visual target consisted of a maximally sized disk that briefly interrupted the smoothly varying size of the disk (Figure 1B). Experiment 2 consisted of a repetition of Experiment 1 with purple visual stimuli instead of red.

\section{Stimuli}

At the patient's position, the auditory looming stimulus varied from 0 to $45 \mathrm{~dB}$ (background noise: $35 \mathrm{~dB}$ ). The patient was seated in a dark room at a distance of approximately $25 \mathrm{~cm}$ from the monitor $(0.2 \mathrm{~cd} / \mathrm{m} 2)$ and a chin rest supported his head. The audiovisual stimuli were prepared as follows: first, an auditory wave of $500 \mathrm{~Hz}$ looming sound of one second was generated with and without target sounds, using GoldWave v.5.68 (GoldWave, Inc). The target sound was generated by inserting the maximum amplitude in the middle of the looming wave time course $(t=500 \mathrm{~ms}$, duration $=10 \mathrm{~ms})$, and synchronized to the middle frame of the video. Next, looming and receding videos were produced in Adobe Flash CS6 (www.adobe.com) and synchronized with the auditory waves resulting in 4 different video files (60 Frame/s) for each of the red and purple colors used [red (colorimetric values: $x=0.60, y=0.35$; luminance $=34 \mathrm{~cd} / \mathrm{m} 2$ ); purple $(x=0.40, y=0.24 ;$ luminance $=35 \mathrm{~cd} / \mathrm{m} 2)]$. At the viewing distance used, the visual looming stimulus varied from $4.8^{\circ}$ to $67^{\circ}$ diameter. The visual receding stimulus was exactly the same as the looming stimulus, only with opposite order of frames in time. Target analogues in looming and receding visual stimuli corresponded to a replacement of the middle frame (31st frame) by a frame containing the largest disk $\left(67^{\circ}\right)$.

To ensure that the purple color was in the wavelength in which the SC is insensitive, we used an established, indirect method (Tamietto et al., 2010), in seven normal participants in a control experiment. Participants were seated at $57 \mathrm{~cm}$ from the screen on which the stimuli were presented. The basic idea of 
the control experiment is that $\mathrm{SC}$ is assumed to provide an RT advantage to bilateral visual targets compared to unilateral targets when stimuli are red, but not when they are purple (given the insensitivity for purple in SC). We used the red and purple colors that were used in our experiment and applied them to the paradigm used by Tamietto et al. (2010), as follows: Red or purple target stimuli (small squares of $2.5^{\circ} \times 2.5^{\circ}$ ) were projected for $200 \mathrm{~ms}$ against a background consisting of other achromatic squares $\left(2.5^{\circ}\right)$ that were changing luminance every $50 \mathrm{~ms}$ (range: $1.1-60 \mathrm{~cd} / \mathrm{m} 2$ ). The background was in view for a variable period prior to the onset of the target(s). Target stimuli were presented on the horizontal meridian to one or both sides of the fixation point (ISI $=3600-4300$ $\mathrm{ms})$, at a distance of $5^{\circ}$ from a central fixation point. There were five randomly intermingled stimulus conditions: single and double red target stimuli, single and double purple target stimuli, and trials without any target stimulus (catch trials). The task of the control participants was to press a button as fast as possible after seeing any stimulus appear on the screen.

\section{Results}

Figure 2 shows the results from the two experiments. Figure 2A (left panel) shows the data for audiovisual learning in Experiment 1, with the darker red trend line corresponding to reaction times to auditory targets that were associated with the visual cue, and the lighter red trend line corresponding to the reaction times to auditory targets that were not associated with the visual cue. The trend lines were generated by a moving average including a window of 6 trials (moved with a step size of one trial). The first 18 trials were excluded from analysis as the patient didn't respond within the 500ms response window. Of the 90 auditory target trials administered in Experiment 1, we therefore kept 44 trials with visually cued auditory targets, and 24 trials with non-cued targets. Reaction times above 500 were excluded, resulting in the first 20 trials of Experiment 1 not being included. The data clearly show a systematic tendency of the reaction times on cued trials (dark red) to be lower than those on noncued trials (light red).

The data in Figure 2A, combined for the two experiments (red: Experiment 1; purple: Experiment 2), show an overall reduction in reaction time that fit well in a power law curve. This suggests an aspecific learning effect, with the patient becoming more skilled in responding to the auditory target. 


\section{Chapter 6}

A

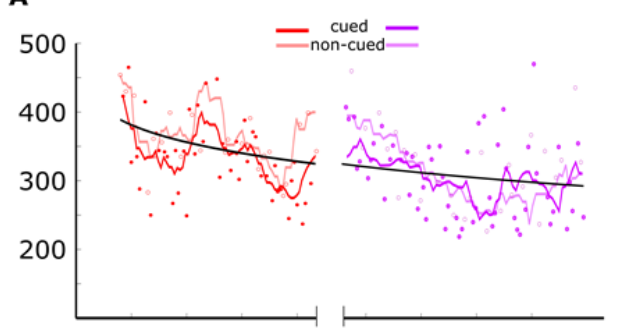

Trial Number
B

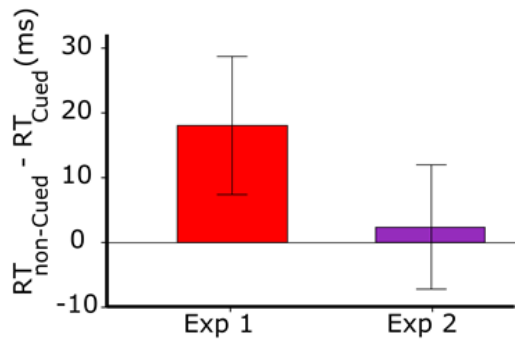

Figure 2 Results of experiments 1 and 2: Red and purple colors are associated to Experiment 1 and 2, respectively (A) Reaction times (RT) as a function of target trial number. The target trial numbers are counted from the beginning of experimental session (full dots: cued trials; empty dots: non-cued trials). The dark lines represent the smoothed RTs of cued trials and the light lines show the smoothed RTs of non-cued trials. (B) Difference between the RT in cued and non-cued conditions for each of the two experiments. The red bar show the average RT difference of the cued trials and noncued trials in each cycle. The error-bars indicate $95 \%$ confidence interval.

To test the differences between cued and non-cued conditions, first, we ensured the normality of the RT difference as the dependent variable. Then, we applied a paired t-test. Cued and non-cued trials were paired based on trialcycle, with each consecutive cycle of 9 trials containing all trial types, including the three cued and non-cued trials. We found a significant difference in reaction time of $36.10 \mathrm{~ms}$ between the cued and non-cued conditions in Experiment 1 ( $\mathrm{t}$ $(23)=3.98 ; p=0.001)$, but no significant difference between the two conditions in Experiment $2(\mathrm{t}(27)=1.65 ; \mathrm{p}=0.11)$.

We also used univariate ANOVA to replicate this analysis, comparing the cued and non-cued trials for each of the two experiments (Figure 2B). The patient was significantly faster in detecting the auditory target when it was visually cued compared to when it was not $(F(1,66)=7.44, p=0.008$, see Figure $2 \mathrm{~B}$, left side). This confirmed the association established between the visual receding stimulus and the target auditory stimulus. An analysis of responses within the response window showed there was no longer any significant difference between the RTs in the visual cued and non-cued conditions $(F(1,81)$ $=0.9, p=0.324$; see Figure $2 B$, right side).

Control experiment. To verify that the colors used in our experiment would show the properties expected for contribution (red) or non-contribution (purple) from the SC, we performed a control experiment, based on the idea that RTs in a speeded reaction time test to two targets shown bilaterally are faster than to a single target shown unilaterally. It has been shown that this 
reaction time advantage disappears when stimuli are made purple (Marzi, Mancini, Metitieri, \& Savazzi, 2009), and hence we replicated this experiment with the specific colors used in our experiment (details in Methods). A paired sample t-test showed significant reduction in reaction time for the bilateral condition compared to the unilateral one for red stimuli but not for the purple ones (7 participants, red: $\mathrm{t}(6)=3.1, \mathrm{p}<0.05$; purple: $\mathrm{t}(6)=0.99, \mathrm{p}=0.36$ ), suggesting that the purple color used in our experiment would indeed lead to a reduction in the contribution of SC to audiovisual association learning.

\section{3| Discussion}

The result from Experiment 1 shows that the patient with complete striate damage can implicitly learn the association between the auditory and the visual stimuli when the stimuli are colored red. We demonstrated in Experiment 2 that the patient can no longer make use of the learned association when the stimuli are rendered purple.

The present study is the first demonstrating the fast development of implicit audiovisual associations (over the course of just a few tens of trials) between consciously perceived auditory stimuli and visual stimuli that cannot be perceived because of complete bilateral striate lesion. The finding that the implicit audiovisual association did not take place for the purple visual stimuli strongly supports the notion that SC plays a critical role in this form of learning. A control experiment in 7 normal participants (details in Methods and Materials) showed a reaction time advantage when responding to two red, compared to one red target in the visual field; an advantage that disappeared with purple stimuli. As the superior colliculus is thought to contribute to this reaction time advantage, these findings support the idea that the purple color used in our visual stimuli indeed could not be processed by the superior colliculus (Tamietto et al., 2010).

Our data are in line with demonstrations of automatic neural audiovisual integration in the anesthetized cat (Yu et al., 2010) and with the anatomical loop between SC and basal ganglia (Redgrave et al., 2010), which may facilitate rapid implicit learning. Furthermore, our result is also consistent with the data from the only other bilateral cortically blind patient studied, who exhibited effective conditioning of startle responses to visual stimuli (Hamm et al., 2003). That finding established another form of implicit learning involving unseen stimuli, likely to depend on extra-genicular visual projections to amygdala and cerebellum (Morris, Ohman, \& Dolan, 1998; Timmann et al., 2010). 


\section{Chapter 6}

The cessation in our study of audiovisual association learning for purple stimuli is consistent with previous research showing the role of SC in unilateral cortically blind patients in various residual visual abilities (Marzi et al., 2009). Our study shows that the extra-genicular pathways not only underlies the residual capacities for visual processing outside awareness, but also contributes to the implicit associative learning between visual stimuli and other relevant information. This opens new perspectives for rehabilitation in cortically blind patients (Boyd \& Winstein, 2006).

\section{Acknowledgements}

This study was partly supported by Human Frontiers Science Program RGP54/2004, NWO Nederlandse Organisatie voor Wetenschappelijk Onderzoek 400.04081, and EU FP6-NEST-COBOL043403 and FP7 TANGO to Beatrice de Gelder, and NWO VICI grant to Peter De Weerd. We would like to thank SJG ten Oever for her suggestions on the design of the experiment. We also thank $M$. Tamietto for his insights on the control experiment.

\section{Conflict of Interest}

The authors declare that there is no conflict of interests regarding the publication of this article. 


\section{References}

Alvarado, J. C., Vaughan, J. W., Stanford, T. R., \& Stein, B. E. (2007). Multisensory versus unisensory integration: contrasting modes in the superior colliculus. J Neurophysiol, 97(5), 3193-3205. doi: 10.1152/jn.00018.2007

Barbur, J. L., Ruddock, K. H., \& Waterfield, V. A. (1980). Human visual responses in the absence of the geniculo-calcarine projection. Brain : a journal of neurology, 103(4), 905-928.

Blythe, I. M., Kennard, C., \& Ruddock, K. H. (1987). Residual vision in patients with retrogeniculate lesions of the visual pathways. Brain : a journal of neurology, 110 (Pt 4), 887-905.

Boyd, Lara A, \& Winstein, Carolee J. (2006). Explicit information interferes with implicit motor learning of both continuous and discrete movement tasks after stroke. Journal of Neurologic Physical Therapy, 30(2), 46-57.

de Gelder, B., Tamietto, M., van Boxtel, G., Goebel, R., Sahraie, A., Van den Stock, J., . . . Pegna, A. (2008). Intact navigation skills after bilateral loss of striate cortex. Current biology, 18(24), R1128-R1129. doi: Pii Sog60-9822(08)01433-4

Hamm, A. O., Weike, A. I., Schupp, H. T., Treig, T., Dressel, A., \& Kessler, C. (2003). Affective blindsight: intact fear conditioning to a visual cue in a cortically blind patient. Brain, 126(Pt 2), 267-275.

Humphrey, Nicholas K. (1974). Vision in a monkey without striate cortex: a case study. Perception, 3(3), 241-255.

Jay, M. F., \& Sparks, D. L. (1984). Auditory receptive fields in primate superior colliculus shift with changes in eye position. Nature, 309(5966), 345347.

Leh, Sandra E, Mullen, Kathy T, \& Ptito, Alain. (2006). Absence of S-cone input in human blindsight following hemispherectomy. European Journal of Neuroscience, 24(10), 2954-2960. 


\section{Chapter 6}

Marzi, C. A., Mancini, F., Metitieri, T., \& Savazzi, S. (2009). Blindsight following visual cortex deafferentation disappears with purple and red stimuli: a case study. Neuropsychologia, 47(5), 1382-1385. doi: 10.1016/j.neuropsychologia.2009.01.023

McHaffie, J.G. , Jiang, H. , Stein, B.E. , \& Willner, W. . (2012). Amelioration of visual orientation deficits in visual cortex lesioned cats by cross-modal behavioral training. . Society Neurosci. Abst.

Meredith, M. A., \& Stein, B. E. (1986). Visual, auditory, and somatosensory convergence on cells in superior colliculus results in multisensory integration. J Neurophysiol, 56(3), 640-662.

Morris, J. S., Ohman, A., \& Dolan, R. J. (1998). Conscious and unconscious emotional learning in the human amygdala. Nature, 393(6684), 467470 .

Pegna, A. J., Khateb, A., Lazeyras, F., \& Seghier, M. L. (2005). Discriminating emotional faces without primary visual cortices involves the right amygdala. Nature neuroscience, 8(1), 24-25.

Pizzamiglio, L., Antonucci, G., \& Francia, A. (1984). Response of the cortically blind hemifields to a moving visual scene. Cortex; a journal devoted to the study of the nervous system and behavior, 20(1), 89-99.

Redgrave, Peter, Coizet, Veronique, Comoli, Eliane, McHaffie, John G, Leriche, Mariana, Vautrelle, Nicolas, . . . Overton, Paul. (2010). Interactions between the midbrain superior colliculus and the basal ganglia. Frontiers in neuroanatomy, 4.

Rosenquist, AC. (1985). Connections of visual cortical areas in the cat. Cerebral cortex, 3, 81-117.

Schiller, P. H., \& Malpeli, J. G. (1977). Properties and tectal projections of monkey retinal ganglion cells. J Neurophysiol, 40(2), 428-445.

Sincich, Lawrence C, \& Horton, Jonathan C. (2005). The circuitry of V1 and V2: integration of color, form, and motion. Annu. Rev. Neurosci., 28, 303326.

Stoerig, P., \& Cowey, A. (1989). Wavelength sensitivity in blindsight. Nature, 342(6252), 916-918. doi: 10.1038/342916ao 
Tamietto, M., Cauda, F., Corazzini, L. L., Savazzi, S., Marzi, C. A., Goebel, R., . . . de Gelder, B. (2010). Collicular vision guides nonconscious behavior. J Cogn Neurosci, 22(5), 888-902. doi: 10.1162/jocn.2009.21225

Timmann, D, Drepper, J, Frings, M, Maschke, M, Richter, S, Gerwig, M, \& Kolb, FP. (2010). The human cerebellum contributes to motor, emotional and cognitive associative learning. A review. Cortex, 46(7), 845-857.

Weiskrantz, L., Warrington, E. K., Sanders, M.D., \& Marshall, J. (1974). Visual capacity in the hemianopic field following a restricted occipital ablation. Brain, 97, 709-728.

Yu, L., Rowland, B. A., \& Stein, B. E. (2010). Initiating the development of multisensory integration by manipulating sensory experience. J Neurosci, 30(14), 4904-4913. doi: 10.1523/JNEUROSCI.5575-09.2010 

CHAPTER 7

\section{COGNITION AND METACOGNITION}

Technical Challenges in Assessing Near-zero Measures of Cognition and Metacognition 


\section{ABSTRACT}

The analysis of near-threshold cognition and metacognition (such as blindsight) is technically complex. In this paper, first, we highlight some of the statistical misconceptions regarding a recent finding called "blind insight". For this, we discuss the analysis methods and findings by Scott et al. (Scott, Dienes, Barrett, Bor, \& Seth, 2014) on the so-called 'blind insight' phenomenon and show the statistical shortcomings of the authors' approach to such findings using their original dataset. Furthermore, we will also discuss the concept of blind (in)sightwhich is originally defined for individuals - in a group analysis and point out the difficulties of such an extension. We believe that the present approach is important for unconscious cognition research in which most of the analyses deal with near-zero cognition and meta-cognition measures.

Keywords: signal detection theory, metacognition, blindsight, blind insight 
In their recent study, Scott et al. (2014) report on a phenomenon they called blind insight based on findings of metacognitive stimulus discrimination when task performance is at chance-level (Scott, Dienes, Barrett, Bor, \& Seth). Participants were asked to classify in a yes-no task whether letter strings conformed to implicitly learned rules (grammar discrimination task) and to express a degree of confidence in their answers (metacognition). Where blindsight reveals that participants may view their performance to be at chancelevel while they are systematically correct, blind insight refers to the ability to judge correctly one's level of performance. They tested 450 participants in a task where the subjects were asked to conform the stimuli to given rules and to rate their answers (type-2 task). The authors focused on a subpopulation of the participants (68 participants; see online supplementary material by Scott et al.) who were below or at the chance-level in the first 3 quarters of the experiment. For their major finding, they also downsized their subpopulation to half due to the incalculable $d_{2}^{\prime}$ in the last quarter of trials, limiting their sample size to 33 participants.

The evidence that metacognition is present even in the absence of discrimination is offered to describe the existence of a new form of metacognitive discrimination. We suggest that this conclusion should be taken with caution in view of the small number of trials per participant reported on in the current study. In particular, we believe that the main limitation of the Scott et al. study is the use of point estimates of $\mathrm{d}_{1}$ to split the tested population into a group performing at chance and one exhibiting discrimination abilities.

Given that only 15-16 trials were used, many participants showed extreme values for hit and false alarm rates, and were excluded from the analysis (infinite $d_{1}^{\prime}$ or $d_{2}^{\prime}$ cannot be used as point estimates). This might in turn lead to an underestimation of variance of the group and the authors' main finding ( $d{ }^{\prime}>0$ "). To address this in more detail, we re-analyze the data of Scott et al. using better measures of metacognition and hierarchical models, resulting in negative findings. We furthermore will show how similar sampling method to select the participants with blindsight-instead of blind insight- would lead to contradictory results.

\section{Is Linear Sampling method justified?}

The statistical assessment of task performance was based on Signal Detection Theory (SDT), which characterizes first-order grammar discrimination accuracy and metacognition with $d_{1}^{\prime}$ and $d_{2}^{\prime}$ respectively. The authors selected a subset of 


\section{Chapter 7}

the participants that exhibited non-positive $d^{\prime}{ }_{1}$ on the first three quarters of the data and showed, on the remaining quarter, that this cohort had metacognitive discrimination ( $d_{2}^{\prime}$ significantly larger than 0 ). They called the above procedure "Linear sampling method". We, first, evaluate such a sampling method.

We believe that the major problem of the paper by Scott et al. is dividing the whole population into two subgroups and having the final conclusion be based on an arbitrary criterion within a subsample (quarter) of trials within that group. Since the $d^{\prime}{ }_{1} s$ and $d^{\prime}{ }_{2} s$ of the entire population seem to come from unimodal distributions, we find it without justification to sample the subjects from the tail of $d^{\prime}{ }_{1}$ distribution to look at their $d_{2}{ }_{2} s$. Moreover, a vast amount of research has proposed acceptable ways to subsample an experimental population, none of which is similar to the so-called linear sampling method, introduced by Scott et al. (Cochran, 2007).

\section{Is there Justification for a Non-unimodal distribution?}

We, first, tested whether there is any evidence from a statistical viewpoint not to believe that the $d^{\prime}{ }_{1} s$ of the entire trials come from a unimodal distribution. We believe that non-unimodality is the key assumption of analysis by Scott et al., permitting them to divide the population into two subgroups. However, they did not mention this.

We examined the distribution (Figure 1) with various unimodality tests. The kurtosis ( $k$ ) and skewness (s) of the distribution are $0.9 \pm 0.1$ and $1.5 \pm 0.2$. As a result, the test value $\left(k^{2}-s^{2}=-1.4 \pm 0.7\right)$ is smaller than $6 / 5$ and $186 / 125$, confirming unimodality based on Rohatgi and Székely(Rohatgi \& Székely, 1989), and Klaassen et al. (Klaassen, Mokveld, \& Van Es, 2000). This test shows that there is no straightforward reason to assume that there is bimodal distribution underlying the $d^{\prime}{ }_{1}$ and $d^{\prime}{ }_{2}$ of the population. We believe that the existence of two subgroups needs to be tested explicitly as it does not seem to be supported by the data. 


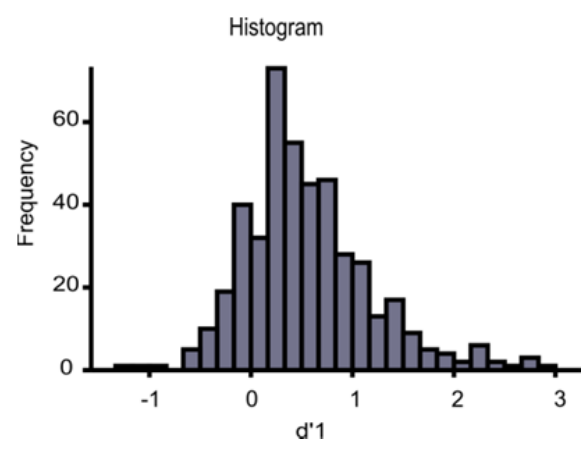

Figure 1 Distribution of $d^{\prime} 1$ in the first 3 quarters of the data across participants.

\section{Resampling of $25 \%$ of the Data}

In light of the foregoing considerations, we show that the small amount of trials in combination with large number of the subgroup with extreme values (i.e. undefinable thus excluded $d_{2}{ }_{2} s$ ) results in a high probability of finding groups with meta-cognition in the last quarter of the data. We estimated the probability of achieving results similar to Scott et al. (d' not larger than 0 and $d^{\prime} 2$ $>0$ ) by randomly drawing the same number of participants as in the blind insight group of Scott et al. (33 subjects) from the last quarter of the trials. The results as depicted in Table 1 - show that in $25 \%$ of cases the results of Scott et al. can be achieved by simply drawing subjects at random in the last quarter of the data.

\begin{tabular}{|c|c|c|}
\hline & $\operatorname{Not}\left(d_{2}^{\prime}{ }^{\text {lastQ }}>0\right)$ & $d_{2}^{\text {lastQ }}>0$ \\
\hline $\operatorname{Not}\left(d_{1}^{\text {lastQ }}>0\right)$ & 0.07 & 0.25 \\
\hline$d_{1}^{\text {lastQ }}>0$ & 0.16 & 0.51 \\
\hline
\end{tabular}

Table 1 the probability of $d^{\prime}{ }_{1}$ and $d^{\prime}{ }_{2}$ of a random selection of 33 participants in the Last quarter of trials reaching significance level $(p<0.05)$ re-sampled 100000 times across all 450 participants. The underlined proportion refers to probability of finding the blind insight phenomenon by random selection of $\mathbf{3 3}$ participants out of $\mathbf{4 5 0}$ in the data from Scott et al.

\section{Insufficient Number of Trials in the Last $1 / 4$}

We furthermore argue that even if two separate subpopulations do exist, the separation criterion used by the authors is difficult to justify. We provide three main arguments demonstrating why $1 / 4$ of trials (15-16 trials) are not believe that the authors should use it to account. 


\section{Chapter 7}

\section{Previous Studies}

Previous research has shown that a reliable $d^{\prime}$ 'value for a participant can only be achieved with sample sizes of at least 50 trials, in the case of near zero d' values (Lee, 2008; Miller, 2000). Furthermore, other authors concluded that a few participants with high number of trials give a much better estimate of the $d$ ' value than a lot of participants with few number of trials (Macmillan \& Creelman, 2004). This is in contrast with the 15-16 trials that were selected by the authors.

\section{Evidence from the First Attempt (50\% Cut-off)}

The authors explicitly mentioned that their first attempt to use the $50 \%$ cut-off value in their linear sampling method did not succeed since the first half of the trials was not a good estimate of the second half due to small sample size. However, they did rely on the $25 \%$ of the final trials as a good estimate of the participant. We find this contradictory that the second half of the data is not representative due to the small sample size but the last quarter is.

\section{Negative $d^{\prime}$}

The other piece of evidence against the reliability of the last quarter comes from the linear sampling method in which they restricted their analysis to the participants with negative $d_{1}^{\prime}$ in the first three quarter of the trials. Theoretically, $d_{1}^{\prime}$ is a non-negative value which is defined as the distance between two underlying distribution functions. In practice, the difference between the transformed hit rates and false alarm rates is an estimate of the true underlying $d_{1}^{\prime}$. In small sample size scenarios, it is crucial to distinguish between the estimated $d_{1}^{\prime}$ as derived from an experiment, and the theoretical value that it estimates. Negative estimates for the within-subject $d_{1}^{\prime}$ have been discussed in the literature as arising from three major sources (Macmillan \& Creelman, 2004). Firstly, negative values may simply result from the underlying distribution of $d^{\prime}$, having a large variance. Naturally, the smaller the number of trials, the larger the resulting observed variability. The common approach towards such near-zero negative $d_{1}^{\prime}$ estimates is to retain them in the group analysis (together with the participants with positive $d_{1}^{\prime} s$ ) to achieve an unbiased estimation of the underlying population $d_{1}^{\prime}$. However, the authors chose only the subjects with negative $d^{\prime}{ }_{1}$ which means the ones who explicitly 
do not have enough trials in their first 40-45 trials. Paradoxically, they did trust the last 15-16 trials of such unreliable participants. We believe that this point independently demonstrates the unreliability of the point estimates, leading to incorrect group analysis. Second, negative $d_{1}^{\prime}$ may arise from systematic confusion as in the case of incorrect associations of the response buttons by some of the participants. This, however does not seem to be the case in the current study because the distribution of observed $d_{1}^{\prime}$ appears unimodal (Figure 2B, top).

A
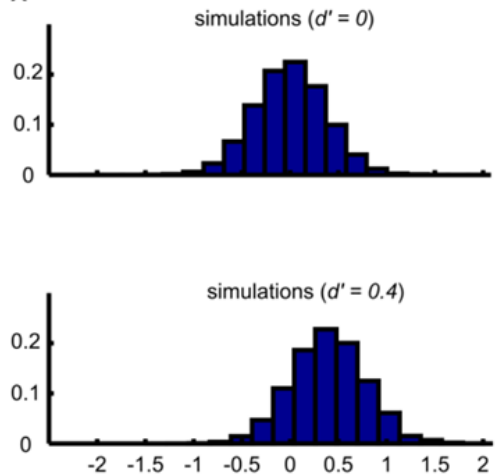

B
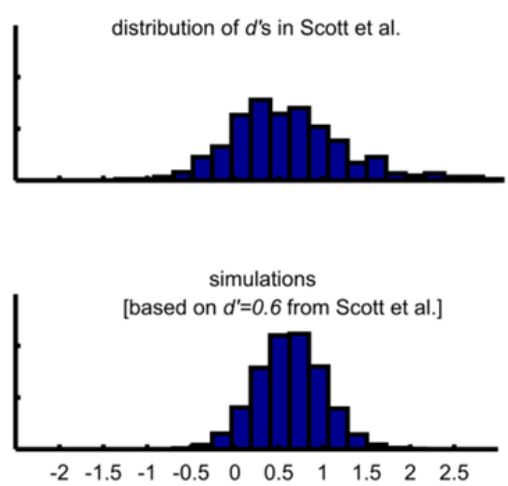

Figure 2 Monte Carlo simulations of $d^{\prime}{ }_{1}$ and their comparisons with the data from Scott et al. (2014): (A) Distributions of $d^{\prime}{ }_{1} s$ resulting from Monte Carlo simulations based on 1 million iterations with true $d^{\prime}{ }_{1}=0$ (top), and true $d^{\prime}{ }_{1}=0.4$ (bottom) for 48 trials. The participant's responses were generated according to a binomial distribution, with error rate for true positives and true negatives similar to Scott et al.; (B) The distribution of $\mathrm{d}^{\prime}{ }_{1} \mathrm{~s}$ in the entire population of the study by Scott et al. (top: 450 participants, reduced to 443 excluding those whose $d^{\prime}{ }_{1}$ could not be calculated; online data set supplemented by the authors); The distribution of simulated $d_{1}{ }_{1}$ with the same sample size and the same mean $\mathrm{d}^{\prime}{ }_{1}$ as in Scott et al., based on 1 million iterations(bottom).

\section{Evidence from the Data Regarding the Between-Subject Variance}

We believe that the use of non-positive $d_{1}^{\prime} s$ to partition the population is inappropriate given the small sample size at the participant level. To further illustrate this point, we performed a set of Monte Carlo simulations with true $d_{1}^{\prime}$ $=0$ and $d_{1}^{\prime}=0.4$ using 48 trials (equal to the three quarter of trials used to determine negative $d_{1}^{\prime}$ by Scott et al.). We performed one million iterations of the simulated experiment where participants' responses were generated according to a binomial distribution, with a similar error rate for true positives 
and true negatives. The results are presented in Figure 2A. The distribution of observed $d_{1}^{\prime}=0$ was centered, as expected, on 0 and its variance was 0.141 (top). The distribution of observed $d_{1}^{\prime}=0.4$ (bottom) showed similar variance (0.143) but it was centered on 0.4. Furthermore, in the latter simulation approximately $17.5 \%$ of the estimated $d_{1}^{\prime}$ were non positive, despite the true $d_{1}^{\prime}$ being 0.4 . This implies that it is possible, with the current sample size, to observe negative $d_{1}^{\prime}$, even in the presence of true discrimination ability.

Based on these considerations, we believe that the procedure used to split the population into the two proposed groups is not statistically justified. Note that the larger variance observed in Scott et al., when compared with a simulation with same sample size and same $d_{1}^{\prime}$ (Figure $2 B$, bottom), could be explained by a single-participant variance combined with additional variance at the population level (a phenomenon known as "over dispersion" in the random effects models literature).

\section{Blindsight: from the Individuals to a Population}

\section{Participants with Blindsight AND Blind Insight at the same time}

As the division into two subpopulations is statistically insufficiently validated, contradictory findings could be obtained from the current dataset: we analyzed the data by Scott et al. using the same criterion but focusing on $d_{2}^{\prime}$ instead of $d_{1}^{\prime}$. We followed the same procedure by selecting the 110 participants with nonpositive $d_{2}^{\prime} \mathrm{s}$ in their first $75 \%$ of trials. Analysis of the last quarter of trials in this subgroup led to above-chance $d_{1}^{\prime}(t(109)=3.06, p=0.003)$. Interestingly, the metacognition in this group was at the chance level in the presence of significant first order accuracy (a condition similar to what is known in the literature as blindsight). We found therefore that, in the same population (450 participants), some participants showed metacognition in the absence of discrimination (110 participants), others showed the opposite effect, and 14 participants showed both. Note that the blind insight population identified by Scott et al. consisted of 33 subjects, thus $42 \%$ of them were blindsight at the same time as well. We believe that these inconsistencies are the outcome of the use of point estimates of $d^{\prime}$, to characterize single participants (Figure 3).

Taken together, we performed the linear sampling strategy used by Scott et al. in order to show that relying on their method can lead to erroneous effects. Our goal was to demonstrate -in logical terms- that the sampling method used by Scott et al. is incorrect, using proof by contradiction (reductio 
ad impossibilem). We showed that if we assume that their sampling method is correct, it immediately leads to a contradiction even within the same dataset of the original research: half of the small blind insight subgroup also belongs to the blindsight group. We believe that this pinpoints a misinterpretation of the blindsight phenomenon in the first place: blindsight is defined as the state of an individual when first-order accuracy takes place in the absence of metacognition.

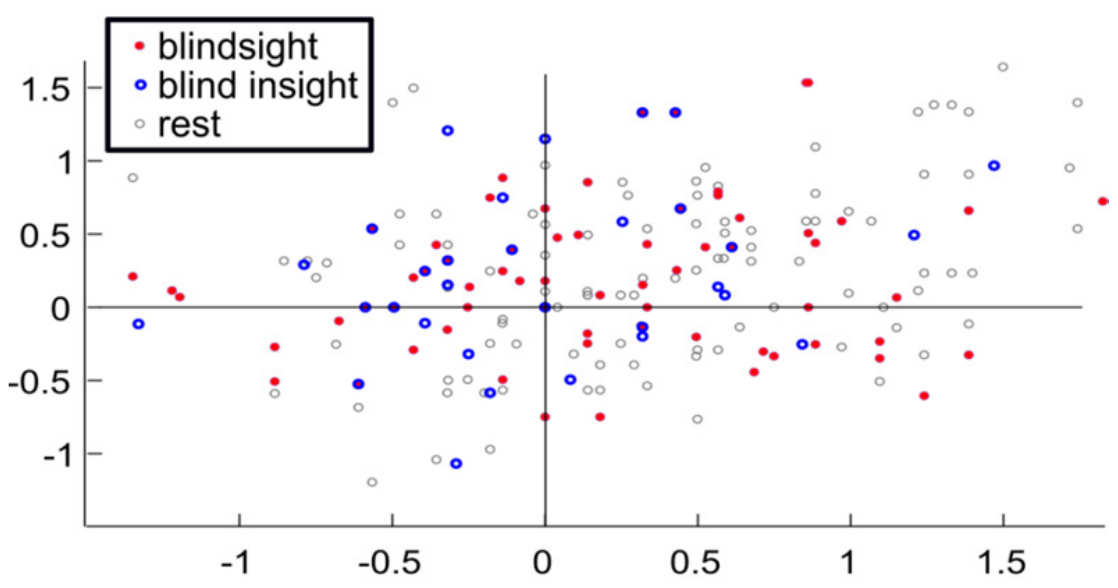

Figure 3 Scatterplot of $d^{\prime} 1$ and $d^{\prime} 2$ in Scott et al. for the last quarter of trials. The blue circles represent the participants who fulfil the blind insight conditions according to the definition by Scott et al. The red circles depict the participants who fulfil the blindsight definition according to Scott et al. The rest of the participants are shown by gray circles. About half of the blind insight individuals also belong to the blindsight group.

\section{Meaning of Blindsight and Blind Insight in a Group}

The extension of individuals' condition to a population requires caution. Figures $4 \mathrm{~A}$ demonstrates the conventional meaning of blindsight and blind insight (B). Figures $4 \mathrm{~B}$ depicts a uniform distribution of $d_{1}^{\prime}$ and $d_{2}^{\prime}$ in a group of hypothetical participants and the definition of blind (in)sight as extended by Scott et al. in a population. They believe that if a subgroup of participants show an average $d_{1}^{\prime}=$ 0 and $d_{2}^{\prime}>0$, the group can be referred to as displaying blindsight. This definition is unprecedented in the literature and we believe that it is not entirely accurate. 
In general, metacognitive measures may reach significantly higher levels than discrimination performance (Charles, Van Opstal, Marti, \& Dehaene, 2013). However, we suggest that, for such a blind insight phenomenon to be supported by the present study, larger data sets - and hence more reliable $d_{1}^{\prime}$ estimates- are required in individual subjects and hence more reliable $d_{1}^{\prime}$ estimates at the individual level.

A

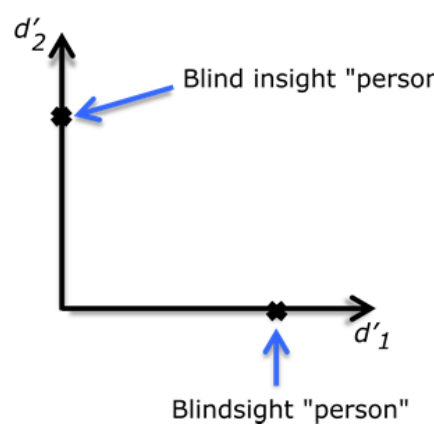

B

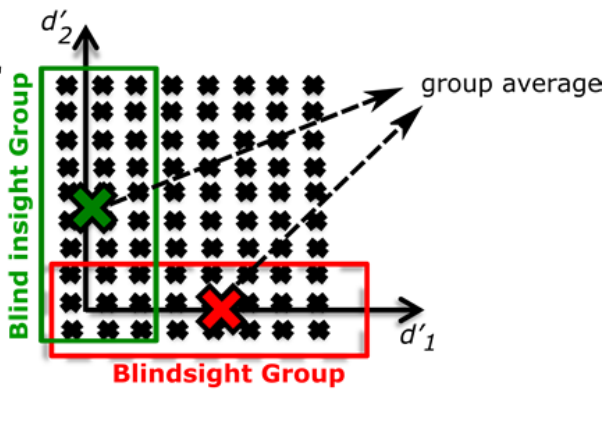

Figure 4 Relationship between $d^{\prime}{ }_{1}$ and $d_{2}^{\prime}$ : (A) the conventional meaning of blindsight and its extension to blind insight; (B) Scott et al.'s interpretation of blindsight and blind insight acquired by group analysis.

\section{4| Alternative Approach for Small Sample Size}

We re-analyzed the data by Scott et al., providing more evidence that the claim " $d_{2}>0$ " in the subpopulation defined using $d_{1}<0$, is not supported by the data, using two approaches. First, we will consider a better measure for metacognition (meta-d') and show how the findings (under the linear sampling model) are not replicated. Then, we will discuss the results of a single subject analysis using more sophisticated statistical models, (hierarchical Bayes) which do not indicate the presence of blind insight as well.

\section{Meta-d' Measure with and without Edge-correction}

Scott et al. performed a supplementary analysis using meta- $d^{\prime}$ instead of $d_{2}^{\prime}$ as meta-d' has recently been discussed to be a better measure of the metacognition performance (Fleming \& Lau, 2014; Maniscalco \& Lau, 2014). The authors claimed that this analysis gave similar results. However, we believe that the analysis was wrongly conducted and a correct use of meta-d' in combination 
with the proposed sampling procedure does not demonstrate the existence of a blind insight subgroup.

The supplementary analysis by Scott et al. using meta-d' was performed on the same group of participants as the original analysis, namely on subjects with non-positive $d_{1}{ }_{1}$ in the first three quarters of the data and calculable $d_{2}^{\prime}$ in the last quarter (68 subjects were identified in the first step, and for 33 of them it was possible to calculate $d_{2}^{\prime}$ ). Importantly, meta- $d^{\prime}$ could be calculated for all the 67 participants with non-positive $d^{\prime}{ }_{1}$, and therefore their procedure is inconsistent: although they aimed to show the same blind insight effect persists with other measures of metacognition, they did not correctly apply their own sampling procedure. Moreover, excluding participants with extreme values would reduce the observed population variance, and thus increase the probability of an unrealistically significant result.

A correct procedure would be, instead, to take into account all the subjects for which meta-d' can be calculated: we therefore re-analyzed the data from Scott et al. using meta-d' instead of $d_{2}^{\prime}$ without discarding participants for which $d_{2}$ c could not be calculated. A common procedure used in meta-d' analysis is to preprocess the data with a procedure called "edge-correction". Edgecorrection approach (representing a "weak" Bayesian approach with a Beta $(1 / 2,1 / 2)$ prior on both hit and false alarm rate) is considered to be more tolerant to the extreme hit rate and false alarm values (Snodgrass \& Corwin, 1988). However, since Scott et al. did not pre-process their data, we performed this analysis both with and without edge-correction. The edge-correction was carried out by the default cell padding parameters in type 2_sdt toolbox (Maniscalco \& Lau, 2012). The summary of the results and more detailed analysis are shown in tables 2 and A2 (Appendix 1) respectively.

\begin{tabular}{|c|c|c|c|c|}
\hline $\begin{array}{c}\text { Metacognition } \\
\text { measure }\end{array}$ & $d_{1}^{\text {, lastQ }}>0$ & $\begin{array}{c}\text { metacognition } \\
>0\end{array}$ & $\begin{array}{c}\text { Metacog } \\
>d^{\prime}{ }_{1}\end{array}$ & $\begin{array}{c}\text { Evidence } \\
\text { for } \\
\text { Blind } \\
\text { insight }\end{array}$ \\
\hline $\begin{array}{c}\text { meta-d' without } \\
\text { edge correction }\end{array}$ & n.s. & n.s. & n.s. & No \\
\hline $\begin{array}{c}\text { meta-d' with } \\
\text { edge correction }\end{array}$ & sig & n.s. & n.s. & No \\
\hline
\end{tabular}

Table 2 Possibility of blind insight within all the participants with ${d^{\prime}}_{1}^{\text {lasta }} \leq 0$ with and without edgecorrection. 
These results clearly indicate that an analysis conducted using meta-d' does not corroborate the hypothesis of existence of a blind insight group. In fact, there is no evidence of metacognition in the last quarter of data with and without edgecorrection. Moreover, when performing edge correction the subjects showing negative $d_{1}$ in the first three quarter have a d'1 significantly larger than 0 in the last quarter.

\section{Hierarchical Bayesian Statistics}

As previously discussed, the blind sight (and hence blind insight) should be shown at single subject level. Given the small number of trials, the comparison of both $d_{1}^{\prime}$ and $d_{2}^{\prime}$ with respect to baseline is clearly underpowered (with few trials, it is difficult to exclude that the observation comes from chance). Moreover, multiple comparison corrections are needed to avoid an inflated false positive rate.

A suitable statistical tool to address this issue is the use of hierarchical models, which integrate both subject specific and population uncertainties. There are many advantages to such procedure: in fact, single subject estimates are more reliable as they integrate evidence provided both by the subject's observation and the population distribution. Moreover, as the subjects are estimated together, the multiple comparison correction is in general not needed (Gelman, Hill, \& Yajima, 2012). In the context of Signal Detection Theory, Bayesian hierarchical models have been introduced to estimate the $d_{1}$ (Lee, 2008; Rouder \& Lu, 2005); however, to the best of our knowledge, no studies have applied the same approach to estimate $d_{2}^{\prime}$ yet. There is only one available (beta) toolbox (Hierarchical Meta-d' Model, HMM) under Matlab for metacognition analysis (https://github.com/smfleming/HMM), which calculates the meta-d' based on a Bayesian framework. However, the proposed model is conditioned on a fixed (deterministic) $d^{\prime}$, and only characterizes probabilistically the type-2 parameters; we believe that a model that can fully estimate all the signal detection parameters in the posterior distribution leads to more realistic estimates.

To achieve the credible intervals on the subject-specific $d_{2}^{\prime}$ values, we extended a recent model by Hyett et al. (2014) by substituting the type-1 hit rates and false alarm rates with the type-2 ones. To estimate the posterior distribution, we use Markov Chain Monte Carlo (MCMC) sampling, which 
provides samples from the posterior distributions of the parameters. We performed Gibbs sampling by JAGS software (http://mcmcjags.sourceforge.net) using 10 chains, 200 burn-ins and 2000 samples per chain. We estimated, per subject, $d_{1}^{\prime}, c_{1}^{\prime}, d_{2}^{\prime}, c_{2}^{\prime}$ (where $c_{1}^{\prime}$ and $c_{2}^{\prime}$ are the type-1 and type-2 biases) from the data by Scott et al. using all trials and all participants.

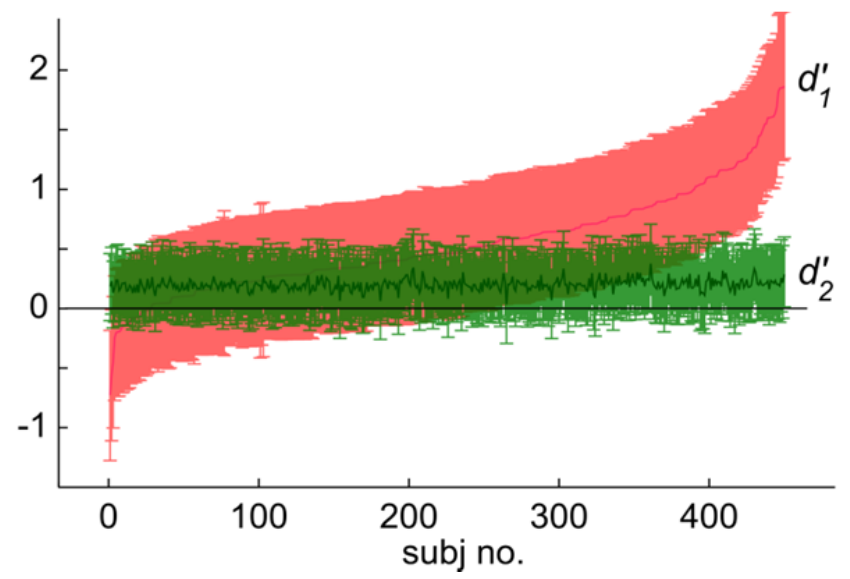

Figure 5 Estimates of $d_{1}{ }_{1}$ and $d^{\prime}{ }_{2}$ based on Bayesian hierarchical model for the data from Scott et al.: Red and green lines represent $d^{\prime}{ }_{1}$ and $d^{\prime}{ }_{2}$ respectively. The participants are sorted ascendingly according to their $d^{\prime}$. Error bars represent the $95 \%$ credible interval achieved by the Bayesian hierarchical model.

The overall results are shown in Figure 5. The subjects are sorted by ascending $d_{1}^{\prime}$, and for each subject the credible interval (and expected value) of the posterior of $d_{1}^{\prime}$ (green) and $d_{2}^{\prime}$ (red) are shown. The results clearly indicate the presence of a considerable number of subjects with d'1 significantly larger than zero $\left(d_{1}^{\prime}=0\right.$ is outside the credible interval), as expected. However, no participant showed the blind insight effect. The difference between the width of credible intervals for $d_{1}^{\prime}$ and $d_{2}^{\prime}$ can be explained by the fact that single subject estimates of $d_{2}^{\prime}$ are much more unreliable than the $d_{1}^{\prime}$ counterpart. Therefore, in the hierarchical model, the single subject estimates are mainly driven by the population estimation. In conclusion, the analysis with a hierarchical model suggests that no subject shows blind insight. 


\section{Appendix 1}

\begin{tabular}{|c|c|c|c|c|c|c|c|c|}
\hline $\begin{array}{l}\text { Metacog } \\
\text { Estimate }\end{array}$ & $\begin{array}{l}\text { Edge- } \\
\text { correct }\end{array}$ & $\begin{array}{c}d f \\
\left(d_{1}{ }^{3 Q}<0\right)\end{array}$ & $\begin{array}{l}\text { Mean } \\
\left(d_{1}^{\text {lasto }}\right)\end{array}$ & $\begin{array}{c}t \\
\left(d_{1}^{\prime}{ }_{1}^{\text {lastQ }}>0\right)\end{array}$ & $\underset{\left(d_{1}^{\text {, lastQ }}>0\right)}{p}$ & $\begin{array}{c}\text { Mean } \\
\text { (Metacog) }\end{array}$ & $\begin{array}{c}t \\
(\text { Metacog }>0)\end{array}$ & $\begin{array}{c}p \\
(m e t a c o g>0)\end{array}$ \\
\hline meta-d' & no & 62 & $0.05 \pm 0.08$ & 0.67 & 0.25 & $-1.1 \pm 0.4$ & -2.37 & 0.99 \\
\hline meta-d' & Yes & 67 & $0.53 \pm 0.20$ & 2.59 & 0.006 & $0.09 \pm 0.07$ & 1.16 & 0.12 \\
\hline
\end{tabular}

Table A1 Detailed reanalysis of all participants with negative $d^{\prime}$ in the first 3 quarter of trials using meta- $d^{\prime}$ as a measure of metacognition with and without edge-correction.

\section{Acknowledgements}

We would like to thank SJG ten Oever and B. Bahrami for reviewing our manuscript.

\section{Conflict of Interest}

The authors declare that there is no conflict of interests regarding the publication of this article. 


\section{References}

Charles, Lucie, Van Opstal, Filip, Marti, Sébastien, \& Dehaene, Stanislas. (2013). Distinct brain mechanisms for conscious versus subliminal error detection. Neuroimage, 73, 80-94.

Fleming, Stephen M, \& Lau, Hakwan C. (2014). How to measure metacognition. Frontiers in human neuroscience, 8.

Gelman, Andrew, Hill, Jennifer, \& Yajima, Masanao. (2012). Why we (usually) don't have to worry about multiple comparisons. Journal of Research on Educational Effectiveness, 5(2), 189-211.

Hyett, Matthew, Parker, Gordon, \& Breakspear, Michael. (2014). Bias and discriminability during emotional signal detection in melancholic depression. BMC psychiatry, 14(1), 122.

Klaassen, Chris AJ, Mokveld, Philip J, \& Van Es, Bert. (2000). Squared skewness minus kurtosis bounded by $186 / 125$ for unimodal distributions. Statistics \& probability letters, 50(2), 131-135.

Lee, Michael D. (2008). BayesSDT: Software for Bayesian inference with signal detection theory. Behavior research methods, 40(2), 450-456.

Macmillan, Neil A, \& Creelman, C Douglas. (2004). Detection theory: A user's guide: Psychology press.

Maniscalco, Brian, \& Lau, Hakwan. (2012). A signal detection theoretic approach for estimating metacognitive sensitivity from confidence ratings. Consciousness and cognition, 21(1), 422-430.

Maniscalco, Brian, \& Lau, Hakwan. (2014). Signal Detection Theory Analysis of Type 1 and Type 2 Data: Meta-d', Response-Specific Meta-d', and the Unequal Variance SDT Model The Cognitive Neuroscience of Metacognition (pp. 25-66): Springer.

Miller, Jeff. (2000). Measurement error in subliminal perception experiments: Simulation analyses of two regression methods. Journal of Experimental Psychology: Human perception and performance, 26(4), 1461. 
Rohatgi, Vijay K, \& Székely, Gábor J. (1989). Sharp inequalities between skewness and kurtosis. Statistics \& probability letters, 8(4), 297-299.

Rouder, Jeffrey N, \& Lu, Jun. (2005). An introduction to Bayesian hierarchical models with an application in the theory of signal detection. Psychonomic bulletin \& review, 12(4), 573-604.

Scott, R. B., Dienes, Z., Barrett, A. B., Bor, D., \& Seth, A. K. (2014). Blind Insight Metacognitive Discrimination Despite Chance Task Performance. Psychological science, 0956797614553944.

Snodgrass, J. G., \& Corwin, J. (1988). Pragmatics of measuring recognition memory: Applications to dementia and amnesia. Journal of experimental psychology: General, 117(1), 34-50. 


CHAPTER 8

\section{GENERAL DISCUSSION AND \\ FUTURE DIRECTIONS}




\section{Chapter 8}

The present dissertation reflects my goal-directed effort in the past few years to answer some of the fundamental aspects of emotion, face and object perception utilizing innovation in psychophysical tools. Moreover -from a technical point of view- I introduced an extension of the adaptation paradigm optimized for high-level vision research. I also presented a novel application for saccadic suppression to be used as a means of research on unconscious processes.

\section{1| Is Face Detection the Gateway for Recognition of Emotional Expressions?}

Humans -since the early days- are used to perceiving a wide range of forms in their natural environment as resembling faces and bodies: from stars to clouds, from rocks to mountains, and even more recently on Mars (Hoagland, 1992). Furthermore, we often associate these shapes with various emotional expressions. Although we are used to experiencing emotional feelings, we are not used to perceiving emotional expressions unaccompanied by human bodies or faces. This intrinsic link between faces and emotional expressions has led scientists in the past decades to build models in which the analysis of emotional expressions was placed within face recognition models. In the present dissertation I questioned this fundamental link.

We all have experienced visual objects and shapes that elevate a certain mood. However, we probably never thought of them acting in the same way as emotional expressions in faces or bodies. At first glance, perceiving an emotional expression without perceiving the shape of a face or body might sound odd and perhaps this is the exact reason why it was never tested in an experimental setup. The entire scientific endeavor to unravel the processing mechanisms of emotional expressions has appeared to take the presence of face stimuli as an assumed prerequisite; i.e. emotion processing occurs always after basic face processing. Furthermore, generating test images of emotional expressions without the presence of a face or body is not a straightforward endeavor. My attempt in the present dissertation is to open the path to revisit some of the conventional views in both face- and emotion research.

The research on emotional expressions dates back to Darwin's theory (Darwin, 1872), which is a lot earlier than the conventional research on face and body perception. However, momentarily most of the experiments and models trying to address the processing stages of emotional expressions -implicitly or explicitly- rely on hierarchical face perception models. Such models include a 
module for the extraction of the emotional expression which follows the core unit of categorizing the stimulus as face. The research in the past 50 years has several times lowered the position of such a process in the hierarchy by showing the independence of processing emotions from other aspects of the face such as identity. Nevertheless, to date the common viewpoint is that the emotional face still gets coded coarsely as a face before entering higher processing units such as emotion recognition.

The first question I tried to answer in the current dissertation is how dissociable face perception and emotion recognition are. My initial attempt was to address this question by means of explicit categorization tasks. Detecting the object as a face is the first building block of hierarchical models of face perception. In the most widely accepted models (Bruce \& Young, 1986; Haxby, Hoffman, \& Gobbini, 2000), face processing starts with a structural description of the stimulus leading to a representation that serves to access the face lexicon. Despite ample of research on the neural correlates of emotional face perception, it is still not clear which brain areas contribute to each of the face perception units proposed by the hierarchical models. The issue of dependency of expression and identity recognition also adds to the ambiguity of the processing stages of face perception. These considerations prompted models of face processing in which faces are represented in at least two partly separate processing streams (de Gelder \& Rouw, 2000; Johnson, 2005). One processing stream is referred to as face detection, which only requires coarse stimulus representation, and the other as face recognition, typically requiring finegrained and elaborate perceptual encoding allowing categorization at a number of levels. All of the later stages of face processing proposed by such models have been investigated intensively in the past thirty years. However, the very first stage which is the necessity of the initial representation of the stimulus as a face has been poorly examined. The initial goal of the present thesis was to investigate whether perception of emotional expression of a face relies necessarily on this gateway or not.

In Chapter 2, I investigated the relation between face detection and emotion categorization by morphing the face in a number of steps towards another object category, and the emotion categorization performance was measured for different emotion-object morphing levels. This was believed to show if there is a need for a high level of face-related information to permit accurate emotion categorization, and if it is possible that the physical information required to categorize the intermediate morphs as face is sufficient to permit emotion categorization. It also envisaged testing whether emotion 


\section{Chapter 8}

categorization can be possible at face-object morph levels below the level required to categorize them as face. The experiment showed that successful categorization of emotions can take place even if the structural information is so little that the stimulus is categorized as a non-face. In other words, the magnitude of the physical threshold for categorizing emotional faces can be moved into the territory of the non-face object category. This can also contribute to the emotional valence of objects we perceive in everyday life.

I further explored this aspect in Chapter 5, knowing that faces presented during saccadic eye movement can barely be perceived consciously, according to findings in Chapter 4. The initial results showed that the barely seen emotional faces presented during saccade cannot be detected as face in a simple face detection task. However, interestingly, the emotional content of such faces was successfully recognized in an emotion categorization task. This is the very first piece of evidence showing that significant emotional processing can occur in the absence of a representation that would allow one to detect the face (or to be aware of it).

\section{Non-hierarchical Models for Face Perception}

The results in Chapters 2 and 5 are in line with the recent advances in the face perception class of models which do not rely on strictly hierarchical stages of processing. The hierarchical models are based on the feedforward hierarchical stages of visual processing, starting with local contours, to simple facial parts in the lower stages of the hierarchy. In the higher stages, such local facial parts integrate to form representations in the face selective areas. As discussed in the introduction, this view is rooted in the early studies and models of early visual cortices (Hubel \& Wiesel, 1962; Marr \& Vaina, 1982; Riesenhuber \& Poggio, 1999; Van Essen, Anderson, \& Felleman, 1992) suggesting local analysis of the visual scene and the subsequent combination of the parts in the higher order processes.

\section{Can the Visual Stimuli Be Processed in the Absence of Primary Visual Cortex?}

In Chapter 6, I presented the findings of our research on the neural pathway involved in learning the associations of the unconsciously processed simple visual stimuli. With a novel audiovisual learning task, we investigated whether the unseen objects can be coded in the complete absence of primary visual cortex. For this, we examined the extent of multimodal learning in the absence 
of the primary visual cortex by carrying out an audiovisual learning experiment on a patient with bilateral damage to striate cortex. The task was to detect an auditory target while (unseen) visual cues were presented on the screen. The results showed implicit learning as evidenced by the patient's shorter reaction times (RTs) to the visually cued stimuli compared to the non-cued ones. We then tested the role of superior colliculus (SC) in such learning behaviors by repeating the same experiment with stimuli shown in purple; a color to which the $\mathrm{SC}$ has been shown to be insensitive. The lack of a visual cueing effect gave us the first evidence for the contribution of SC to implicit audiovisual learning. Taken together the results showed that audiovisual association learning can take place not only in the absence of conscious perception of the stimuli but even in the complete absence of the primary visual cortex through a subcortical route including SC.

\section{2 | Technical Developments}

The secondary emphasis of the present thesis is to introduce more advanced psychophysics tools for studying high level vision. In Chapter 3, I presented a novel extension of the conventional adaptation paradigm which relies on the known properties of high level processes.

\section{Suppression of Complex Objects during Saccadic Eye Movements}

One of the main purposes of my research in the last few years was to establish a method to reliably suppress face processing at its earliest stage which is the detection of the stimulus as a face. There are already a few established methods to suppress the visibility of faces and other stimuli. The most important ones are backward masking and continuous flash suppression. However, both of these methods introduce serious confounding factors - such as indissociable effect of the target and the mask - making it difficult to make strong conclusions about the exact underlying mechanism. Moreover, faces due to their high saliency- are even more difficult to be entirely suppressed from the conscious vision at the early processing stages. For example one of the major studies investigating the neural correlate of unconscious perception of fearful expressions is a research by Whalen et al. (2004). They showed that unconscious processing of fearful expressions heightens amygdala activity. Summarized, the authors briefly presented eye regions -instead of the whole faces- which conveyed emotional expressions, and immediately masked them 


\section{Chapter 8}

with neutral faces. This led to suppression of the participants' awareness about the emotional stimuli. They showed the unseen fearful stimuli still activated the amygdala. However, they did actually not acquire an objective measure of the viewers' awareness about the masked stimuli. More importantly their design was not apt to measure the face detection accuracy for such suppressed emotional stimuli.

\section{Audiovisual Association Learning}

The design presented in Chapter 6 for audiovisual learning is novel. Most of the research in the field of blindsight has been focused on 2-alternative-forcedchoice (2AFC) tasks as the measure of performance. Some recent studies, however, also take into account RT as the other indication of performance change in the blindsight patients, only focusing on the redundant target effect (RTE) (Marzi, Tassinari, Aglioti, \& Lutzemberger, 1986; Tamietto et al., 2010; Tomaiuolo, Ptito, Marzi, Paus, \& Ptito, 1997). I believe that comparing RTs in audiovisual association learning tasks in the presented format can provide an additional tool in the experiments targeting the non-conscious processes.

\section{Technical Difficulties Regarding Near-Zero Sensitivities}

During the course of research on unconscious perception, I encountered serious difficulties in quantifying subjects' performance reliably at close to chance level regime. I realized that this problem is not limited to a small subset of blindsight studies, but several other studies have made substantial misinterpretation of the concepts together with statistical deficits. Specifically, a recent research claiming the existence of "blind insight" was in my opinion highly flawed. The authors had used a series of analytical methods to reach their conclusions claiming that participants at chance for a simple discrimination task would be significantly successful in the corresponding type-2 tasks to detect their own errors.

\section{3| Future Directions}

The future direction of the research presented in this manuscript can be divided into two categories: 


\section{Short-term Prospective}

Mid-/High-level vision. The novel adaptation paradigm proposed in Chapter 3 to assess the mid-high level adaptation effects can be used with the advanced imaging techniques such as functional magnetic resonanceadaptation ( $\mathrm{fMRI}-\mathrm{a})$. This can provide a better understanding of the underlying mechanisms of the high-level processes. Especially, the added value of this method to the conventional fMRI-a method is achieving a behavioral measure of the mid/high-level adaptation effect which is absent in most of the present $\mathrm{fMRI}-\mathrm{a}$ experiments. Correlating the behavioral measure to the $\mathrm{fMRI}$-a signal provides a much more selective task-relevant activity.

Neural correlates of audiovisual association learning. The novel design presented in Chapter 6 for audiovisual learning can also be used in combination with functional imaging techniques in order to reveal the detailed neural correlates of audiovisual association learning both in normal population and in the patients with lesions in their striate cortex.

Bayesian statistics for d' analysis. Signal detection theory (SDT) as a mathematical tool for unbiased analysis was first introduced in the field of electronics (Peterson, Birdsall, \& Fox, 1954), where the radars needed to discern between the signal and the noise. It was immediately adopted by the psychophysicists (Tanner Jr \& Swets, 1954) as an efficient means of acquiring unbiased behavioral measures. With certain considerations, SDT approach often works flawlessly for measuring the cognition and metacognition measures in individuals. However, the extension of such measures to a population is not always straightforward. The common approach in the usage of SDT for group analysis is to take the number of trials as high as possible for the within-subject SDT analysis in order to acquire the sensitivity and bias point estimates for every participant with very little error. The next step in this approach is to apply the classical statistics such as analysis of variance (ANOVA), which normally rely on strong assumptions about the distribution of the data. In the past few years, there is a large tendency to move towards the Bayesian statistics which is basically data driven and not dependent on the assumptions about the data distributions. Furthermore, few researchers have started developing Bayesian approach towards SDT in the past few years (Lee, 2008), which are restricted to the single-subject SDT measures so far. Single-subject Bayes-SDT is potentially significant for the future research. However, I believe that the major challenges 


\section{Chapter 8}

of SDT takes place while conclusions are to be made for the group of participants. Nonetheless, there is no such analytical tool taking into account both within- and between-subject variability. Regarding this shortage, one of the important future extensions of this work is to provide a free online toolbox for Bayesian analysis of signal detection theory for group analysis.

\section{Long-term Prospective}

Computer vision and robotics. This thesis provides the first evidence for an early separation of face representations for the recognition of facial emotions (e.g., fear versus happy) from face representations for basic-level categorization (i.e., face versus other objects). A better understanding of how our visual system extracts emotions from a face is theoretically important, and I therefore believe that our findings can give a new direction to both computational modelling efforts and imaging/neurophysiological investigations of emotion perception. In addition, this new direction of research may lead to new applications in the domain of face screening and monitoring, as well as robotics and even marketing. Our findings are relevant for all of the aforementioned domains of investigation.

Therapeutic and educational applications of unconscious training. Unraveling the aspects of unconscious perception and unconscious learning in the present dissertation is not only useful to discover how face and object perception work, but can contribute insight to research into specific types of implicit learning. For example, unconscious cues can be used to improve certain skills in the normal population such as executive control (Capa, Bustin, Cleeremans, \& Hansenne, 2015). Furthermore, such implicit learnings can as well help the patients with brain lesions to improve their vision (Das, Tadin, \& Huxlin, 2014; Sahraie, 2014). Moreover, this approach in the long run can also be implemented to improve certain behavioral conditions such as addiction, eating disorder, etc. (Harris, Bargh, \& Brownell, 2009; Leventhal et al., 2008). 


\section{References}

Bruce, V., \& Young, A. W. (1986). Understanding face recognition. British journal of Psychology, 77, 305-327.

Capa, Rémi L, Bustin, Gaëlle M, Cleeremans, Axel, \& Hansenne, Michel. (2015). Conscious and unconscious reward cues can affect a critical component of executive control. Experimental psychology.

Darwin, C. R. . (1872). The expression of the emotions in man and animals (First edition ed.). London: John Murray.

Das, Anasuya, Tadin, Duje, \& Huxlin, Krystel R. (2014). Beyond blindsight: properties of visual relearning in cortically blind fields. The Journal of neuroscience, 34(35), 11652-11664.

de Gelder, B., \& Rouw, R. (2000). Structural encoding precludes recognition of parts in prosopagnosia. Cognitive Neuropsychology, 17, 89-102.

Harris, Jennifer L, Bargh, John A, \& Brownell, Kelly D. (2009). Priming effects of television food advertising on eating behavior. Health Psychology, 28(4), 404 .

Haxby, J. V., Hoffman, E. A., \& Gobbini, M. I. (2000). The distributed human neural system for face perception. Trends in cognitive sciences, 4(6), 223-233.

Hoagland, Richard C. (1992). The monuments of Mars: a city on the edge of forever (Vol. 2800): North Atlantic Books.

Hubel, D. H., \& Wiesel, T. N. (1962). Receptive fields, binocular interaction and functional architecture in the cat's visual cortex. J Physiol, 160, 106-154.

Johnson, M. H. (2005). Subcortical face processing. Nature reviews. Neuroscience, 6(10), 766-774. doi: 10.1038/nrn1766

Lee, Michael D. (2008). BayesSDT: Software for Bayesian inference with signal detection theory. Behavior research methods, 40(2), 450-456.

Leventhal, Adam M, Waters, Andrew J, Breitmeyer, Bruno G, Miller, Elizabeth K, Tapia, Evelina, \& Li, Yisheng. (2008). Subliminal processing of smoking- 


\section{Chapter 8}

related and affective stimuli in tobacco addiction. Experimental and clinical psychopharmacology, 16(4), 301.

Marr, David, \& Vaina, Lucia. (1982). Representation and recognition of the movements of shapes. Proceedings of the Royal Society of London. Series B. Biological Sciences, 214(1197), 501-524.

Marzi, CA, Tassinari, G, Aglioti, S, \& Lutzemberger, L. (1986). Spatial summation across the vertical meridian in hemianopics: A test of blindsight. Neuropsychologia, 24(6), 749-758.

Peterson, W Wesley, Birdsall, Theodore Gerald, \& Fox, We. (1954). The theory of signal detectability. Information Theory, Transactions of the IRE Professional Group on, 4(4), 171-212.

Riesenhuber, M., \& Poggio, T. (1999). Hierarchical models of object recognition in cortex. Nature neuroscience, 2(11), 1019-1025. doi: 10.1038/14819

Sahraie, Arash. (2014). Vision exercising apparatus: Google Patents.

Tamietto, M., Cauda, F., Corazzini, L. L., Savazzi, S., Marzi, C. A., Goebel, R., ... de Gelder, B. (2010). Collicular vision guides nonconscious behavior. J Cogn Neurosci, 22(5), 888-902. doi: 10.1162/jocn.2009.21225

Tanner Jr, Wilson P, \& Swets, John A. (1954). A decision-making theory of visual detection. Psychological review, 61(6), 401.

Tomaiuolo, F, Ptito, M, Marzi, CA, Paus, T, \& Ptito, A. (1997). Blindsight in hemispherectomized patients as revealed by spatial summation across the vertical meridian. Brain, 120(5), 795-803.

Van Essen, D. C., Anderson, C. H., \& Felleman, D. J. (1992). Information processing in the primate visual system: an integrated systems perspective. Science, 255(5043), 419-423.

Whalen, P. J., Kagan, J., Cook, R. G., Davis, F. C., Kim, H., Polis, S., ... Johnstone, T. (2004). Human amygdala responsivity to masked fearful eye whites. Science, 306(5704), 2061. 


SUMMARY 
How we distinguish faces from other objects ("face detection") and how we extract the emotional signal from other people faces ("emotion recognition") are still open questions. Most conventional models for face perception are proposing a hierarchical relationship between these stages in which face detection is the entry point for all further processing stages including emotion recognition. There is an increasing amount of evidence that processing of emotional expressions takes place separately from the other high-level processing stages of faces such as person identification. However, no other research has addressed if extracting the emotional information can be independent of the most primary processing stage of the current models which is detecting the stimulus as a face. This book is presenting the first evidence that emotion recognition can take place independent of face detection; in other words, we can recognize the facial expression without being able to detect the object as face in certain conditions.

In Chapter 2, the relationship between the face detection and emotion recognition was investigated in the context of explicit categorization of morphed stimuli between an object and emotional faces. For this, the explicit categorization of morphed stimuli between non-face objects and emotional faces were tested. The results showed that the underlying emotion information was still available for the stimuli that were categorized as non-face, suggesting a certain degree of separation between the two processes.

In Chapter 3, the effect of recent experience -known as adaptation- on face and object detection was tested. For this, the performance changes in face and nonface detection tasks due to recent exposure to face and non-face stimuli were measured. The results interestingly showed that face detection can be deteriorated by recent exposure to both faces and non-face objects, whereby object detection can only be influenced by adaptation to the same object category.

In Chapter 4 a new method for suppressing the face detection performance was introduced. The method was based on the saccadic suppression phenomenon which corresponds to the reduced visual awareness during fast eye movements (known as saccades). Then the same method was used in Chapter 5 to investigate if any emotion recognition can take place in the case of complete unawareness of the face stimuli where face detection is at chance. The results showed that the unconscious categorization abilities of the participants for such unseen faces were significantly above chance in emotion 
categorization while the face detection task was at chance. This finding draws a serious doubt on the most commonly accepted models of face perception and suggests a much higher degree of independence between processing of faces within the face recognition framework and their emotion content.

Chapters 6 and 7 were focused on other aspects of unconscious perception. Chapter 6 presented a study on audiovisual association learning abilities in a human patient with the rare condition of a bilateral cortical lesion to primary visual cortex. The results showed that learning the association of the sound and the (unseen) visual stimuli can take place in the absence of the primary visual cortex. Furthermore, the results suggested that this effect was mediated by superior colliculus. Chapter 7 underlines some technical difficulties in unconscious perception research by addressing the difficulties of a recent study on metacognition abilities (the ability to distinguish between one's correct and incorrect responses). The analysis of the original data showed that the authors' findings were due to small sample size and inappropriate extension of the meaning of blindsight and "blind insight" to the group level. 



\section{KNOWLEDGE VALORIZATION}




\section{Relevance}

Understanding how the brain functions is one of the most fundamental questions contributing to almost all fields of science, technology and philosophy. One of the basic science branches addressing this question is cognitive neuroscience which tries to answer the most ancient problems of mind and behavior. Thus, limiting the scope of any of its findings to a few shortterm practical matters is undermining the importance of such a research. In other words, the very first goal of the current work should be viewed as any other basic research, which might or might not contribute to the near, far, or very distant future regardless of any direct implication in business and society.

This book reflects my endeavor in the past few years investigating some of the most fundamental neural coding mechanisms used by the unconscious mind through emotions and social interactions. The majority of the current research aims at uncovering the coding scheme for emotional faces in the brain. Faces are the most universal social cues that are common across cultures and countries. As a result the bottom-line is that the findings of this thesis can be extended to the whole world population. Moreover, Understanding how the emotions are extracted from the visual scene is of great theoretical and practical importance. It also gives new directions to computational modeling of the visual system and brain imaging research on emotion perception. Such directions can lead to new technologies of face recognition in machines. One simple application of such technologies is in face monitoring for security purposes.

\section{To whom are the Research Results of Interest?}

What are emotions and why do we need them? This has been an ancient central question in life sciences, philosophy, education, economics and many other fields of research. Thus, our better understanding of how the brain processes the emotion-relevant information in an almost real-time fashion is of great interest for the scientists in all the aforementioned fields, from machine learning to economics, and from education to marketing.

Moreover, the audiovisual blindsight study in Chapter 6 demonstrates that the patients with complete loss of primary visual cortex can learn the associations between the sounds and the stimuli presented in their blind visual field. The study was carried out in an extremely rare case of complete striate 
cortex lesion. In the first sight one might think that the findings are very limited to that small group of patients. However, looking more closely at the main findings reveals that the blindsight study in fact suggests the possibility of such audiovisual learning in the absolute absence of primary visual cortex suggesting that various types of (cortical) visual dysfunctions might be improved by pairing the visual and the auditory stimuli. This finding can lead new rehabilitation technologies for patients with various visual difficulties.

\section{Innovation}

The present work can interest a broad range of audience. On one side I have presented some novel practical paradigms to measure the human behavior. Measuring certain aspects of unconscious perception is one of the most complex tasks a behavioral scientist might face. The methods and paradigms I presented in this book would interest a wide range of scientists involved in measuring human behavior in different fields of experimental psychology, marketing, brain imaging, behavioral economy, marketing research, etc. Furthermore, devices aiming at inducing audio-visual learning in the patients with various visual disabilities can be one of the natural continuations of the present work.

\section{Potential Products and Services}

\section{Computer Vision and Robotics}

As mentioned before, the core finding of this thesis on unconscious processing of the emotional faces is potentially important in computer vision and robotics. Especially, it can lead to improved machine learning algorithms aiming at detecting human faces, bodies and their emotions in the realistic environment and processing them rapidly.

\section{Therapeutic applications}

Discovering various aspects of unconscious-perception and learning in the present dissertation is not only beneficial in the basic understanding about the neural codes of such processes, but can also contribute to novel therapeutic developments for patients with various neurological or psychiatric disorders. For example, I showed in chapter 6 that the patients with lesion in striate cortex can learn the association of the auditory signals to the visual stimuli. 


\section{Valorization}

Computer-based therapies based on such implicit learnings can help the patients with different anomalies to improve their vision.

\section{Bayesian Statistics in Signal Detection Analysis}

As discussed in chapter 7, we developed a computational method to improve the precision of Signal Detection Theory (SDT) in the small-sample size regime. One of the possible future products is an easy-to-use toolbox based on our current software accessible to all scientists who do similar analyses in the small sample-size regime. By this, they will be able to achieve more reliable statistical measures of their studies.

\section{Short-term Applications}

\section{Imaging Studies}

The novel adaptation paradigm in chapter 3 can inspire new neuroimaging studies aiming at behavioral changes due to adaptation. So far, most of such studies (in the field of fMRI-adaptation) solely focus at the changes in the BOLD activity- as an estimate of the underlying neural activity- regardless of the participants' actual behavioral correspondence. In the next one year, we are able to update the present method to be used in the fMRI scanner and find more selectively underscore the subtle changes in the BOLD activity.

\section{Neural Correlates of Audiovisual Association Learning}

The novel design presented in Chapter 6 for audiovisual learning enables a new range of $f M R I$ studies targeting the neural correlates of audiovisual association learning both in patients and normal controls.

\section{Signal Detection Analysis (SDT)}

From a computational point of view, one of the most sophisticated behavioral measures the scientists can use is SDT. In chapter 7, I showed that using Bayesian statistics in SDT, taking into account both within- and between-subject variabilities will lead to more reliable results. Regarding the shortage of an all-inone toolbox for such analysis, one of the main short-term goals in the next one year will be to develop an open-access freeware for such analyses. 


\section{Long-term Applications}

\section{Computer Vision and Robotics}

Object recognition, is still one of the most important unresolved problems in machine learning. This means that we still cannot make devices, smart enough to recognize objects and faces flawlessly. As mentioned before, one of the goals in understanding the underlying mechanisms of perception is to apply similar algorithms (and probably the hardware) to accomplish face and object recognition at the performance level comparable to that of the humans. There is no definite timing for how long it might take until we reach that stage, but we can be sure any research in the field of human perception is a step ahead in this field.

\section{Therapeutic Applications}

Another emphasis of the current work is focusing on a novel approach to improve the conditions in cortically blind patients. In the long run, we can use the current framework to apply audiovisual associations for the unseen objects through the sounds in such patients. This has a great potential of significantly improving the quality of life for such patients. 

ACKNOWLEDGEMENTS 
Thanking the people who have been significantly helpful to me in the past years of pursuing the $\mathrm{PhD}$ is too complicated. The problem is that I have been gifted so enormously in the last few years that thanking every person who deserves acknowledgement would need another book to be written! I try to be concise.

First of all, thanks to my PhD supervisors Bea and Peter. I specially thank Bea, the one without whom this whole PhD would never take place. I also thank Peter who showed me that super hard-core scientists are not extinct yet. The most constant support has been done by my lovely parents and my gorgeous sisters Maryam and Marjan. I also thank the light of my house, mijn Assaltje, Sanne, who has been "de beste" in the past three years.

The most influential event in my life has been starting improvisation theatre with a group of super cool people! Improvisation theatre not only changed my view on life but also improved my problem-solving and presentation skills. Too many members to call but few to be named are: Gabri, Nevena, Jessie, Paola, Jan, Joanne, Despina, Peter, Dorjin, Eveline, Mare, Zubin, Bas, Latifa, Viktorija, Lukasz, Alan, Norbert, Masha, Paul, Francesco, Mari, Nordin, Burcu, Yeliz, Anna, etc.

Special thanks also to my great friend and paranymph Gojko, with who has been my mate since the beginning of our master's program. Also thanks to my other paranymph, Paola, with her amazing south Italian warmth. Not only for all the nice times during our improvisation sessions but also for the directing the best low-budget movie ever!

The other circle made the duration of my PhD even more pleasant was the small but warm group of my compatriots: Ehsan, Nastaran, Ehsan, Saba, Ali,

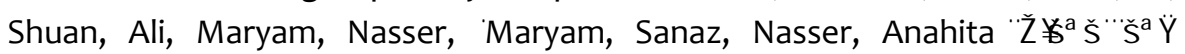

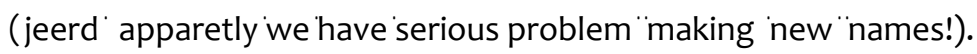

From the Cognitive Neuroscience department, I would like to thank the constant support of Kamil probably the best head a department can have! I also thank the faculty members who taught me more than just science during my studies: Bernadette the most amazing dean a faculty can imagine; Rainer, who showed me how a person can be a successful businessman while remaining as a real scientist; Elia who is by far the smartest person I met in Maastricht; Alex with a great ambition for technical challenges and novel techniques; Joel with his high precision in details; and Giancarlo for his deep 
computational understanding and modest attitude helping everybody with all kinds of technical problems. I also thank Hanneke and Pascal for supporting me during the harshest times of seemly unresolvable problem. Furthermore, I thank Valerie for being the only person in the department with whom I could talk about my research in the beginning of my $\mathrm{PhD}$ while she was still in Maastricht.

I thank my office-mates who coped with me in the last couple of years: Marin, Bert, Arne and Mario. I also thank all the PhD students and postdocs at Faculty of Psychology and Neuroscience who supported me during this time: Vittoria, Rebecca, Nathalie, Julia, Joao, Lars, Tobi and many others. Also, very special thanks to Faruk for his genius skills to improve the quality of the cover page of this dissertation significantly in a couple minutes and Rosanne for her artistic input on my design. I also thank the members of Bea's lab including, Minye, Lisanne, Ruud, Aline, Mariska, Bernard, Kiki, Tahnee, etc. I also thank members of Peter's group including Gesa, Mark, Alex, etc.

I would also like to thank all the rest of the PhD academy board members: Daan, Alejandro, Elisa, Alisson (and many others to be named!). PhD academy is for sure the best thing that could happen for the PhD candidates in Maastricht.

I would also like to thank all the actors and crews of "For the Love of Science". It was a great pleasure sharing time with you guys! I also thank all the members of the Swing Dance Community, PhD Dance, and SwingOut Maastricht for their enthusiasm and passion to make the scene happen from the scratch in this city; among others: Caterina, Karen, Georgina, Hendrik, etc.

I also thank the members of "II Desastro", Ali, Livia, Geraldin, etc. for their warm hospitalities and the great food. I enjoyed every moment at their fun parties. I also thank Kirsten and Dennis with whom I spent splendid days and nights, talking about science, Syria, fish and lizards! Furthermore, I thank all the other members of the International Dinner (whom I haven't named so far) for their great hospitalities and delicious food including Thes, Martin, Jo, Yara, Paula, Giacomo, Jullie, Joost, Sean, Artemis, Cheng, Elisa, etc.

Special thanks to all those came from far places for my defense including Taha and Mehrnaz. I also thank the support of all my friends in the 
European institutes in Maastricht, including, Greta, Francesco, Quentin, Adrian, Anna, Sean, Fabien, Anna, Paola, Matteo, etc.

Moreover, I would like to thank Vincent, Richard and all the other members of Applied Biomedical Systems for their support in the last couple of months. I especially thank Vincent for giving me all kinds of mental support in establishing and growing EmoSys. Finally, thanks to Fabien, Nico and the rest of the staff at Brightlands Health Campus for their unassuming support. 




\section{PUBLICATIONS AND \\ CURRICULUM VITAE}





\section{Journal Articles}

Seirafi, Mehrdad, Peter De Weerd, and Beatrice L. de Gelder. "Emotion categorization does not depend on explicit face categorization." Journal of vision 13.2 (2013): 12.

Seirafi, Mehrdad, Peter De Weerd, and Beatrice de Gelder. "Suppression of Face Perception during Saccadic Eye Movements." Journal of ophthalmology 2014 (2014).

\section{Under Review:}

Seirafi, Mehrdad, Peter De Weerd, Alain Pegna, and Beatrice de Gelder. "Audiovisual association learning in the absence of primary visual cortex".

Seirafi, Mehrdad, Giancarlo Valente, Peter De Weerd, and Beatrice de Gelder. "Blind sight and blind insight: How small sample sizes can affect metacognition analysis".

Seirafi, Mehrdad, Peter De Weerd, and Beatrice L. de Gelder. "Attribute selective adaptation paradigm: Category-specific effect of adaptation on faces and objects".

In preparation:

Seirafi, Mehrdad, Peter De Weerd, and Beatrice L. de Gelder. "Faceless Emotions Emotion categorization in the absence of face detection". 


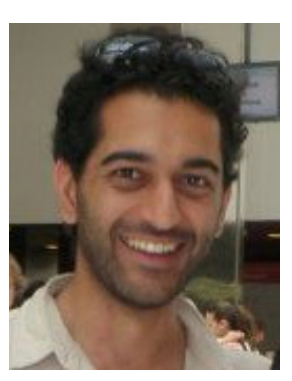

Mehrdad Seirafi was born on March 21, 1982 in Tehran, Iran. He obtained Bachelor of Science in Physics at Sharif University of Technology, Tehran (2005). He later received his Master of Science in Cognitive Neuroscience from Maastricht University (2010). In the meanwhile, he gained research experience in Abdus Salam Center for Theoretical Physics (Italy), Tata Institute for Fundamental Research (India), RIKEN Brain Institute (Japan), School of Cognitive Sciences (Iran), and Tilburg University (the Netherlands). During his PhD, he was awarded as the winner of Science Slam Maastricht (2013). He also received the Faculty of Psychology and Neuroscience (FPN) research day best oral presentation award (2013). In February 2015, he established EmoSys, a company designing wearable devices for mood elevation. 